\title{
Solid Phase Synthesis of (Benzannelated) Six- membered Heterocycles via Cyclative Cleavage of Resin-bound Pseudo-oxazolones
}

Simone Gräßle, ${ }^{a}$ Sylvia Vanderheiden, ${ }^{b}$ Patrick Hodapp, ${ }^{a}$ Bekir Bulat ${ }^{\mathrm{a}, 5}$, Martin Nieger ${ }^{c}$, Nicole Jung, ${ }^{a, b}$ * and Stefan Bräse ${ }^{a, b}$ *

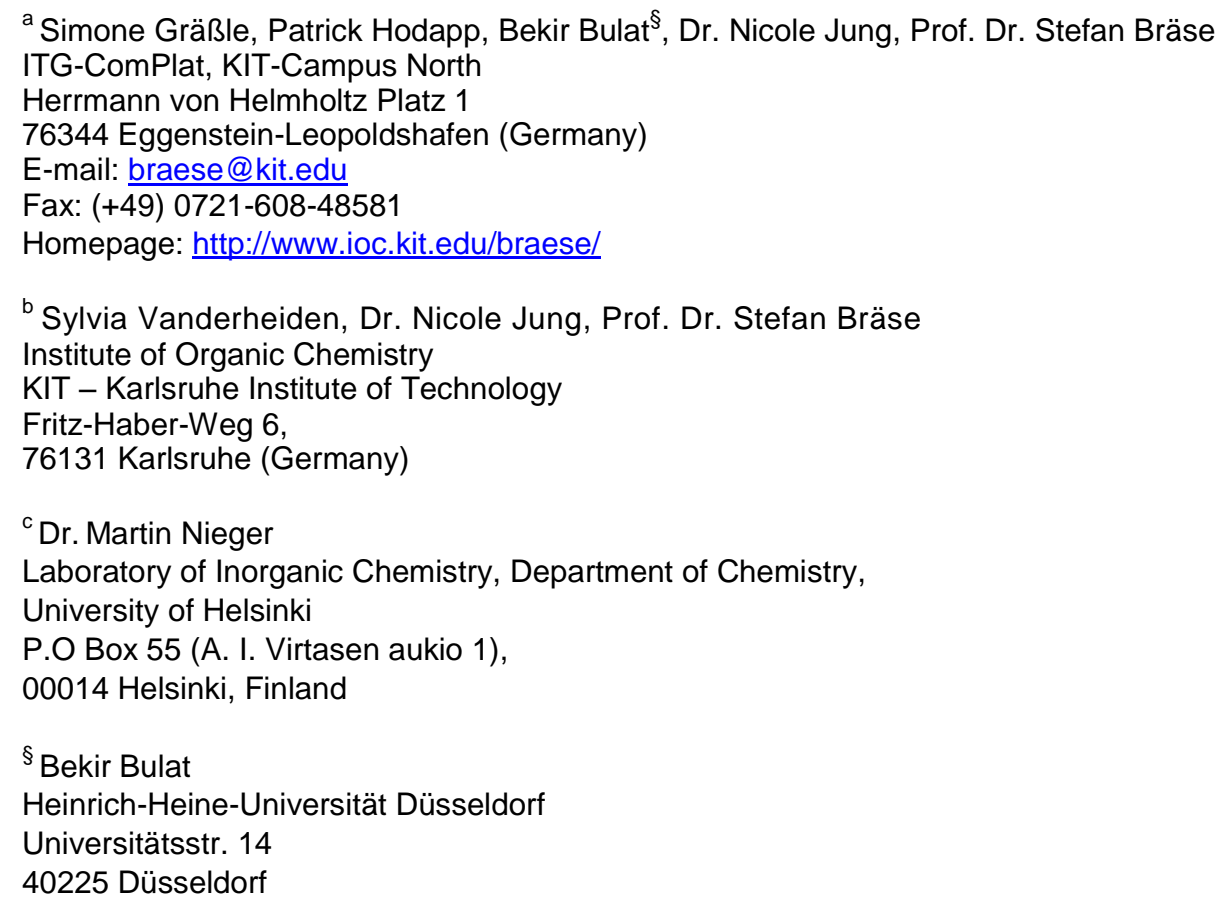

\section{Contents}

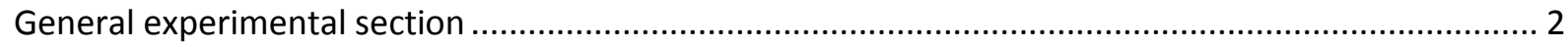

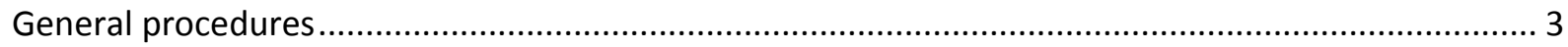

General washing procedure (given per $1.00 \mathrm{~g}$ of crude resin) ........................................................ 3

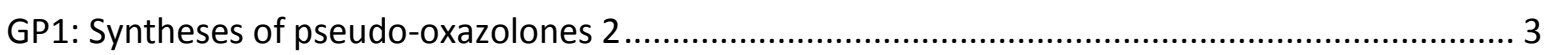

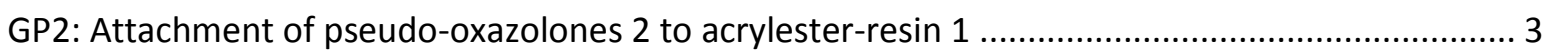

GP3: Cleavage of oxazolone-containing resins 3 to dihydropyrazineones and quinoxalinones .......... 3

GP4: Cleavage of Oxazolone-containing resins 3 to benzo[1,4]oxazin-2-ones and dihydro-1,4-

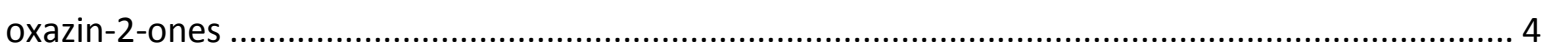

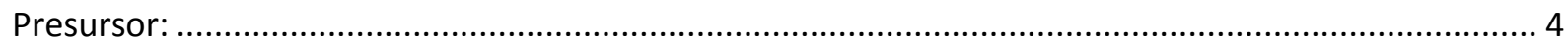

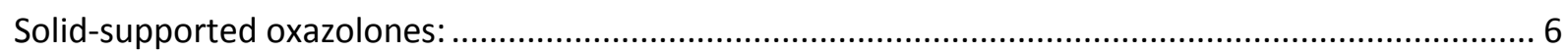

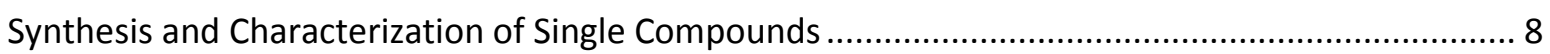

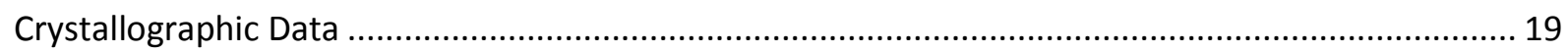

Spectra 


\section{General experimental section}

${ }^{1}$ H NMR spectra were recorded on a Bruker AM 300 (300 MHz), a Bruker AM 400 (400 MHz), and a Bruker AM $500(500 \mathrm{MHz})$ spectrometer. Chemical shifts are given in parts per million $(\delta / \mathrm{ppm})$, downfield from tetramethylsilane (TMS) and are referenced to chloroform (7.26 ppm), methanol (3.31 $\mathrm{ppm})$ or dimethylsulfoxide (2.50 ppm) as internal standards. All coupling constants are absolute values and $\mathbf{J}$ values are expressed in Hertz $(\mathrm{Hz})$. The description of signals include: $\mathrm{s}=$ singlet, $\mathrm{bs}=$ broad singlet, $\mathrm{d}=$ doublet, $\mathrm{bd}=$ broad doublet, $\mathrm{t}=$ triplet, $\mathrm{dd}=$ doublet of doublets, $\mathrm{dt}=$ doublet of triplets, $\mathrm{m}$ $=$ multiplet. The spectra were analyzed according to first order. ${ }^{13} \mathbf{C}$ NMR spectra were recorded on Bruker AM $300(75 \mathrm{MHz})$, Bruker AM $400(100 \mathrm{MHz})$ and Bruker AM $500(125 \mathrm{MHz})$ spectrometers. Chemical shifts are expressed in parts per million $(\delta / \mathrm{ppm})$ downfield from tetramethylsilane (TMS) and are referenced to chloroform $(77.4 \mathrm{ppm})$, methanol $(49.15 \mathrm{ppm})$ or dimethylsulfoxide (39.5 ppm) as internal standards. MS (EI) (electron ionization mass spectrometry): Finnigan MAT $90(70 \mathrm{eV})$. The molecular fragments are quoted as the relation between mass and charge $(\mathrm{m} / \mathrm{z})$, the intensities as a percentage value relative to the intensity of the base signal $(100 \%)$. The abbreviation $[\mathrm{M}]^{+}$refers to the molecule ion. IR (infrared spectroscopy): ATR spectra were recorded by diamond crystal on Bruker ALPHA-IR.

Routine monitoring of reactions were performed using silica gel coated aluminium plates (Merck, silica gel 60, F254) which were analyzed under UV-light at $254 \mathrm{~nm}$ and/or dipped into a solution of molybdato phosphate (5\% phosphor molybdic acid in ethanol, dipping solution) and heated with a heat gun. Solvent mixtures are understood as v/v. Solvents, reagents and chemicals were purchased from Sigma Aldrich, Alfa Aesar, ABCR and VWR. Solvents, reagents and chemicals were used as purchased unless stated otherwise. Merrifield resin was purchased from Polymer Laboratories (PLCMS resin $0.94 \mathrm{mmol} / \mathrm{g}$ or PL-CMS resin $0.99 \mathrm{mmol} / \mathrm{g}, 75-150 \mu \mathrm{m}, 1 \%$ cross linkage).

Calculation of yield according to change of mass of the washed and dried resins:

mass difference expected $[\mathrm{mg}]=$ change $\operatorname{molar}$ mass $[\mathrm{g} / \mathrm{mmol}] \mathbf{x}$ mmol $_{\text {start }}$ mass difference $_{\text {found }}[\mathrm{mg}]=\operatorname{mass}_{\text {new }}[\mathrm{mg}]-\operatorname{mass}_{\text {old }}[\mathrm{mg}]$

(1) If mass difference found $_{2} \geq$ mass difference expected, $_{\text {, }}$ then:

assumed yield $=100 \%$

loading $_{\text {new }}=\frac{\text { mmol starting }[\mathrm{mmol}]}{\operatorname{mass}[\mathrm{g}]}$
(2) If mass difference found $\leq$ mass difference expected, $_{\text {, }}$ then:

$$
\begin{aligned}
& \text { yield }=\frac{\text { mass difference found }}{\text { mass difference expected }} \\
& \text { loading }_{\text {new }}=\frac{\text { mmol starting }[\mathrm{mmol}] \mathbf{x} \text { yield }}{\text { mass }[\mathrm{g}]}
\end{aligned}
$$

Change of molar mass: The mass difference induced by substitution of the starting material with a novel fragment (e.g. substitution of $\mathrm{Cl}$ against first building block in case of Merrifield resin as starting material). 


\section{General procedures}

\section{General washing procedure (given per $1.00 \mathrm{~g}$ of crude resin)}

The resin was washed successively with $30 \mathrm{~mL}$ of dichloromethane $(2 \mathrm{x}), 30 \mathrm{~mL}$ of acetone $(2 \mathrm{x}), 30 \mathrm{~mL}$ of methanol (2x), $30 \mathrm{~mL}$ of DMF, $30 \mathrm{~mL}$ of water $(2 x), 30 \mathrm{~mL}$ of methanol $(2 \mathrm{x}), 30 \mathrm{~mL}$ of dichloromethane $(2 x)$.

\section{GP1: Syntheses of pseudo-oxazolones 2}<smiles>[R]C(N)C(=O)O</smiles>

AS<smiles>[R]C1=NC(C(F)(F)F)OC1=O</smiles>

2

In a typical procedure, the amino acid (AS) (1.00 equiv.) was dissolved in trifluoroacetic acid anhydride (TFAA) (4.00 equiv.) and was heated for $2 \mathrm{~h}$ at $60{ }^{\circ} \mathrm{C}$. The remaining TFAA was removed via distillation and the target compound was isolated either via distillation (alanine) or extraction (all other AS) and optional column chromatography. The procedure for the extraction was as follows: The reaction mixture was diluted with saturated aqueous $\mathrm{Na}_{2} \mathrm{CO}_{3}$ solution and diethylether and the organic phases were collected. The aqueous layer was extracted three times with diethylether. The combined organic layers were washed twice with aqueous $\mathrm{Na}_{2} \mathrm{CO}_{3}$-solution and brine. The organic layers have been dried over $\mathrm{Na}_{2} \mathrm{SO}_{4}$ and the solvent was removed via evaporation. The crude material was purified via flash chromatography to give the pure compounds for analytics.

GP2: Attachment of pseudo-oxazolones 2 to acrylester-resin 1

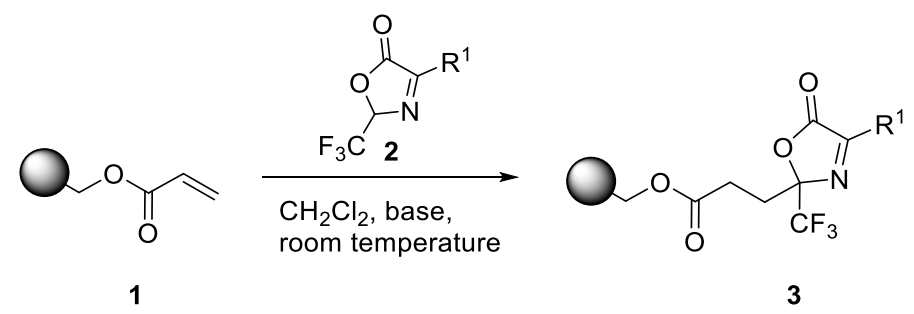

Acrylester resin 1 (1.00 equiv.) was added to a solution of 2-(trifluoromethyl)oxazol-5(2H)-one (3.504.50 equiv.) and $\mathrm{NEt}_{3}$ (Procedure GP2A, 3.00-4.00 equiv.) or DMAP (Procedure GP2B, 3.00 4.00 equiv.) in $20.0 \mathrm{~mL} / \mathrm{g}$ of $\mathrm{CH}_{2} \mathrm{Cl}_{2}$ and was shaken for $16 \mathrm{~h}$ at room temperature. The resin was collected via filtration, purified according to general washing procedure and dried under high vacuum.

\section{GP3: Cleavage of oxazolone-containing resins 3 to dihydropyrazineones and quinoxalinones}

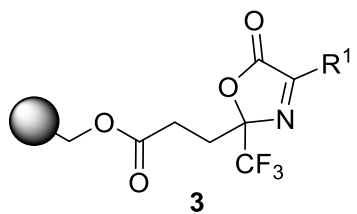

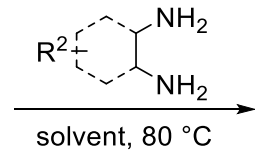

solvent, $80^{\circ} \mathrm{C}$<smiles>[R1]C1=NC2CC[R1]CC2NC1=O</smiles>

5

If not otherwise stated in the description of the single molecule, the resin was suspended in $\mathrm{MeOH}$ (min. of $10 \mathrm{~mL}$ of solvent per $1 \mathrm{~g}$ of resin) and 3.00 equiv. of diamine was given to the mixture. The mixture was heated to $80^{\circ} \mathrm{C}$ at this temperature for $15 \mathrm{~h}$ if not otherwise stated and the resin was removed via filtration. The filtrate was collected, evaporated and the crude material was purified via flash chromatography to give the pure compounds. 
GP4: Cleavage of Oxazolone-containing resins 3 to benzo[1,4]oxazin-2-ones and dihydro-1,4oxazin-2-ones<smiles>[R]C1=NC(CCC(=O)OC=O)(C(F)(F)F)OC1=O</smiles><smiles>[R12]CC(C)C1CCCCC1N</smiles><smiles>[R]C1=NC2CC[R1]CC2OC1=O</smiles>

If not otherwise stated in the description of the single molecule, the resin was suspended in $\mathrm{MeOH}$ (min. of $10 \mathrm{~mL}$ of solvent per $1 \mathrm{~g}$ of resin) and 3.00 equiv. of 1,2-aminoalcohol were given to the mixture. The mixture was heated to $80^{\circ} \mathrm{C}$ and reacted at this temperature for $16 \mathrm{~h}$ if not otherwise stated and the resin was removed via filtration. The filtrate was collected, evaporated and the crude material was purified via flash chromatography to give the pure compounds.

\section{Presursor:}

\section{Polystyrenemethyl acrylate (1)}<smiles>C=CC(=O)OC=O</smiles>

Merrifield resin $(5.00 \mathrm{~g}$, loading: $0.940 \mathrm{mmol} / \mathrm{g}, 4.70 \mathrm{mmol})$ was swollen in $50 \mathrm{~mL}$ of DMF and $1.74 \mathrm{~g}$ of acrylic acid $\left(1.66 \mathrm{~mL}, 24.1 \mathrm{mmol}, 5.13\right.$ equiv.), $7.56 \mathrm{~g}$ of $\mathrm{Cs}_{2} \mathrm{CO}_{3}$ (23.2 mmol, 4.94 equiv.) and $385 \mathrm{mg}$ of $\mathrm{KI}(2.32 \mathrm{mmol}, 0.49$ equiv.) were added. The reaction mixture was heated to $80^{\circ} \mathrm{C}$ and was shaken for $15 \mathrm{~h}$. After purification according to general washing procedure, $5.42 \mathrm{~g}$ of the white target resin 1 were obtained in quantitative yield (loading: $0.867 \mathrm{mmol} / \mathrm{g})$. The reaction has been performed several times and the yields are summarized in the table given below. $\left.{ }^{13} \mathrm{C} \mathrm{Gel-NMR} \mathrm{(100} \mathrm{MHz,} \mathrm{CDCl} 3, \mathrm{ppm}\right), \delta=66.3\left(\mathrm{CH}_{2} \mathrm{O}\right), 166.0$ (CO). IR (ATR, v): 3024, $2919,1723,1600,1492,1450,1404,1266,1175,1027,964,808,753,695,536 \mathrm{~cm}^{-1}$.

Table of loading:

\begin{tabular}{cccccc} 
No & start.amount[g] & mmol & yield $[\mathrm{g}]$ & conversion & loading[mmol/g] \\
\hline $\mathbf{1 . 1}$ & 5.00 & 4.70 & 5.42 & quant & 0.867 \\
$\mathbf{1 . 2}$ & 20.9 & 20.7 & 21.6 & quant & 0.956 \\
$\mathbf{1 . 3}$ & 20.9 & 21.1 & 21.6 & $98 \%$ & 0.960 \\
$\mathbf{1 . 4}$ & 20.7 & 19.5 & 21.5 & quant & 0.907 \\
$\mathbf{1 . 5}$ & 25.3 & 25.0 & 26.2 & quant & 0.956 \\
$\mathbf{1 . 6}$ & 24.9 & 24.6 & 25.8 & quant & 0.927 \\
\hline
\end{tabular}

\section{4-Methyl-2-(trifluoromethyl)oxazol-5(2H)-one (2a).}<smiles>CC1=NC(C(F)(F)F)OC1=O</smiles>

According to General Procedure 1, alanine $(15.0 \mathrm{~g}, 168 \mathrm{mmol})$ was reacted with TFAA (95.0 mL, $673 \mathrm{mmol}, 4.00$ equiv.) for $2 \mathrm{~h}$ at $60^{\circ} \mathrm{C}$. TFAA was removed via distillation and the target compound was isolated via vacuum distillation $\left(25 \mathrm{mbar}, 60^{\circ} \mathrm{C}\right)$ to give $11.6 \mathrm{~g}(69.4 \mathrm{mmol})$ of the target compound in $41 \%$ yield as a clear liquid. ${ }^{1} \mathrm{H}$ NMR (300 $\left.\mathrm{MHz}, \mathrm{CDCl}_{3}, \mathrm{ppm}\right), \delta=2.38$ (d, $\left.J=2.3 \mathrm{~Hz}, 3 \mathrm{H}\right), 6.08-6.14(\mathrm{~m}, 1 \mathrm{H}) .{ }^{13} \mathrm{C}$ NMR $(75 \mathrm{MHz}$, $\left.\mathrm{CDCl}_{3}, \mathrm{ppm}\right), \delta=13.9,92.9(\mathrm{q}, J=36.1 \mathrm{~Hz}), 120.1(\mathrm{q}, J=280 \mathrm{~Hz}), 164.0,166.4 .{ }^{19} \mathrm{~F} \mathrm{NMR}(376 \mathrm{MHz}$, $\left.\mathrm{CDCl}_{3}, \mathrm{ppm}\right) \delta=-79.02 . \mathrm{El}\left(\mathrm{m} / \mathrm{z}, 70 \mathrm{eV}, 100{ }^{\circ} \mathrm{C}\right): 167$ (8) [M] ${ }^{+}, 140$ (49), 122 (7), 69 (100). HRMS $\left(\mathrm{C}_{8} \mathrm{H}_{10} \mathrm{~F}_{3} \mathrm{NO}_{2}\right)\left[\mathrm{M}^{+}\right]$: calc. 167.0189, found 167.0188. IR (ATR, v): 1801, 1655, 1371, 1319, 1271, 1189, $1151,1102,1016,976,870,750,699,633,532,415 \mathrm{~cm}^{-1}$. 


\section{4-(4-Hydroxyphenyl)-2-(trifluoromethyl)oxazol-5(2H)-one (2b).}<smiles>O=C1OC(C(F)(F)F)N=C1c1ccc(O)cc1</smiles>

According to General Procedure 1, 4-hydroxyphenylglycine (1.00 g, 5.98 mmol) was converted in TFAA (5.03 g, $3.38 \mathrm{~mL}, 23.9 \mathrm{mmol}, 4.00$ equiv.) to give the crude target compound in $96 \%(1.41 \mathrm{~g}, 5.76 \mathrm{mmol})$ yield. After purification via column chromatopraphy $(\mathrm{CH}: \mathrm{EE}, 4: 1 \rightarrow 2: 1)$, the pure compound was obtained in $86 \%$ yield as a white solid. $R_{f}=0.26$ (Cyclohexane:Ethylacetate, 4:1). ${ }^{1} \mathrm{H}$ NMR $\left(250 \mathrm{MHz}, \mathrm{CDCl}_{3}, \mathrm{ppm}\right), \delta=3.72(\mathrm{~s}, 1 \mathrm{H}), 6.19(\mathrm{q}, J=4.1 \mathrm{~Hz}$, $1 \mathrm{H}), 6.81-6.90(\mathrm{~m}, 2 \mathrm{H}), 8.19-8.30(\mathrm{~m}, 2 \mathrm{H}) .{ }^{13} \mathrm{C} \mathrm{NMR}\left(63 \mathrm{MHz}, \mathrm{CDCl}_{3}, \mathrm{ppm}\right), \delta=92.0(\mathrm{q}, J=35.0 \mathrm{~Hz})$, $115.9,118.8,120.2$ (q, $J=282.5 \mathrm{~Hz}), 131.2,159.3,162.5,163.4 .{ }^{19} \mathrm{~F} \mathrm{NMR}\left(376 \mathrm{MHz}, \mathrm{CDCl}_{3}, \mathrm{ppm}\right)$ $\delta=-78.79$. El (m/z, $\left.70 \mathrm{eV}, 50{ }^{\circ} \mathrm{C}\right): 245(100)[\mathrm{M}]^{+}, 201(43), 120$ (72). HRMS $\left(\mathrm{C}_{10} \mathrm{H}_{6} \mathrm{~F}_{3} \mathrm{NO}_{3}\right)\left[\mathrm{M}^{+}\right]$: calc. 245.0294, found 245.0293. IR (ATR, ṽ): 3316, 2463, 1805, 1774, 1600, 1576, 1515, 1440, 1369, 1398, $1261,1217,1160,1131,1050,1021,973,896,843,700,649,623,535,470,421 \mathrm{~cm}^{-1}$. EA $\left(\mathrm{C}_{10} \mathrm{H}_{6} \mathrm{~F}_{3} \mathrm{NO}_{3}\right)$ : calc. C 48.99, H 2.47, N 5.71; found. C 48.80, H 2.19, N 5.67.

\section{4-Phenyl-2-(trifluoromethyl)oxazol-5(2H)-one (2c).}<smiles>O=C1OC(C(F)(F)F)N=C1c1ccccc1</smiles>

2-Amino-2-phenylacetic acid $(5.10 \mathrm{~g}, 33.7 \mathrm{mmol}, 1 \mathrm{eq})$ was dissolved in trifluoroacetic anhydride $\left(28.3 \mathrm{~g}, 19.1 \mathrm{ml}, 135 \mathrm{mmol}, 4.00\right.$ equiv.) at $0^{\circ} \mathrm{C}$. The reaction mixture was stirred at $60{ }^{\circ} \mathrm{C}$ for $2 \mathrm{~h}$, then neutralized with $\mathrm{Na}_{2} \mathrm{CO}_{3}$ and extracted 3 times with diethyl ether. The combined organic phases were washed with $1 \mathrm{~N} \mathrm{HCl}$, neutralized with sat. $\mathrm{NaHCO}_{3}$, washed with brine and the solvent removed under reduced pressure. The product was isolated as a white solid in $91 \%$ yield (7.05 g, $30.8 \mathrm{mmol}$ ) as a white solid. $\mathrm{R}_{f}$ (cyclohexane:ethyl acetate, $\left.2: 1\right)=0.77 .{ }^{1} \mathrm{H} \mathrm{NMR}(250 \mathrm{MHz}$, $\left.\mathrm{CDCl}_{3}, \mathrm{ppm}\right), \delta=6.28(\mathrm{q}, J=4.1 \mathrm{~Hz}, 1 \mathrm{H}), 7.51-7.59(\mathrm{~m}, 2 \mathrm{H}), 7.63-7.71(\mathrm{~m}, 1 \mathrm{H}), 8.40-8.49(\mathrm{~m}, 2 \mathrm{H})$. ${ }^{13} \mathrm{C} \mathrm{NMR}\left(63 \mathrm{MHz}, \mathrm{CDCl}_{3}, \mathrm{ppm}\right), \delta=92.3(\mathrm{q}, J=35.4 \mathrm{~Hz}), 120.3(\mathrm{q}, J=283.0 \mathrm{~Hz}), 129.0,129.1,134.0$, 160.6, 162.7, 171.2. $\left.{ }^{19} \mathrm{~F} \mathrm{NMR} \mathrm{(376} \mathrm{MHz,} \mathrm{CDCl} 3, \mathrm{ppm}\right) \delta=-78.62 . \mathrm{El}\left(\mathrm{m} / \mathrm{z}, 70 \mathrm{eV}, 30{ }^{\circ} \mathrm{C}\right): 229$ (100) [M] $]^{+}$, 185, 104, 77. HRMS $\left(\mathrm{C}_{10} \mathrm{H}_{6} \mathrm{~F}_{3} \mathrm{NO}_{2}\right)$ : calc. 229.0345, found 229.0344. IR (ATR, v): 1791, 1610, 1572, 1495, 1452, 1374, 1335, 1304, 1278, 1193, 1142, 1078, 1044, 1001, 968, 866, 802, 746, 701, 685, $668,526,435,402 \mathrm{~cm}^{-1}$. EA $\left(\mathrm{C}_{10} \mathrm{H}_{6} \mathrm{~F}_{3} \mathrm{NO}_{2}\right)$ : calc. C 52.41, H 2.64, N 6.11; found. C 52.67, H 2.25, N 6.11.

\section{4-Methyl-2-(trifluoromethyl)oxazol-5(2H)-one (2d).}

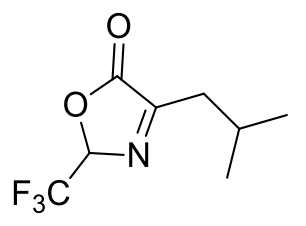

Leucine $(2,50 \mathrm{~g}, 19,1 \mathrm{mmol}, 1 \mathrm{eq})$ was dissolved in trifluoroacetic anhydride $\left(16.0 \mathrm{~g}, 10.8 \mathrm{ml}, 76.0 \mathrm{mmol}, 4.00\right.$ equiv.) at $0{ }^{\circ} \mathrm{C}$. The reaction mixture was stirred at $60{ }^{\circ} \mathrm{C}$ for $2 \mathrm{~h}$ then poured on ice and extracted with diethyl ether. The combined organic phases were washed with sat. $\mathrm{Na}_{2} \mathrm{CO}_{3}$ until the all acid was neutralized, then washed with brine and the solvent removed under reduced pressure. A clear liquid was isolated in $82 \%$ yield $(3.26 \mathrm{~g}, 15.6 \mathrm{mmol})$.

$\mathrm{R}_{f}$ (Cyclohexane:Ethylacetate, 2:1) $=0.72$. Chromatography in cyclohexane:ethyl acetate $(10: 1 \rightarrow$ 4:1). ${ }^{1} \mathrm{H} \mathrm{NMR}\left(300 \mathrm{MHz}, \mathrm{CDCl}_{3}, \mathrm{ppm}\right), \delta=1.00(2 \mathrm{xd}, J=6.7,6 \mathrm{H}), 2.24(\mathrm{dt}, J=13.4 \mathrm{~Hz}, J=6.7 \mathrm{~Hz}, 1 \mathrm{H})$, $2.48-2.68(\mathrm{~m}, 2 \mathrm{H}), 3.54-3.61(\mathrm{~m}, 1 \mathrm{H}), 6.07-6.17(\mathrm{~m}, 1 \mathrm{H}) .{ }^{13} \mathrm{C} \mathrm{NMR}\left(63 \mathrm{MHz}, \mathrm{CDCl}_{3}, \mathrm{ppm}\right), \delta=22.2$ $(\mathrm{d}, J=8.5 \mathrm{~Hz}), 26.2,36.7,93.0(\mathrm{q}, J=35.6 \mathrm{~Hz}), 120.1(\mathrm{q}, J=281 \mathrm{~Hz}), 163.9,168.5 .{ }^{19} \mathrm{~F} \mathrm{NMR}(471 \mathrm{MHz}$, $\left.\mathrm{CDCl}_{3}, \mathrm{ppm}\right) \delta=-78.97$. El (m/z, $\left.70 \mathrm{eV}, 30^{\circ} \mathrm{C}\right): 209[\mathrm{M}]^{+}, 194,182,166,140,131,119,69$ (100). HRMS $\left(\mathrm{C}_{8} \mathrm{H}_{10} \mathrm{~F}_{3} \mathrm{NO}_{2}\right)\left[\mathrm{M}^{+}\right]$: calc. 209.0658, found 209.0658. IR (ATR, v): 2965, 1803, 1647, 1468, 1372, 1318, $1271,1191,1154,1081,1015,951,870,700,673,533,477 \mathrm{~cm}^{-1}$. EA $\left(\mathrm{C}_{8} \mathrm{H}_{10} \mathrm{~F}_{3} \mathrm{NO}_{2}\right)$ : calc. C 45.94, $\mathrm{H}$ 4.82, N 6.70; found. C 46.12, H 4.48, N 6.73 . 


\section{2,2,2-Trifluoro-N-(3-(5-oxo-2-(trifluoromethyl)-2,5-dihydrooxazol-4-yl)propyl)acetamide (2e).}<smiles>O=C1OC(C(F)(F)F)N=C1CCCNC(=O)C(F)(F)F</smiles>

In a $50 \mathrm{~mL}$ round-bottomed flask 2,5-diaminopentanoic acid $(2.50 \mathrm{~g}$, $18.9 \mathrm{mmol}, 1 \mathrm{eq})$ was dissolved in trifluoroacetic anhydride $(15.9 \mathrm{~g}$, $10.7 \mathrm{ml}, 76.0 \mathrm{mmol}, 4 \mathrm{eq})$ at $0^{\circ} \mathrm{C}$. The reaction mixture was stirred at $60{ }^{\circ} \mathrm{C}$ for $2 \mathrm{~h}$ then poured on ice and extracted with diethyl ether. The combined organic phases were washed with sat. $\mathrm{Na}_{2} \mathrm{CO}_{3}$ until the all acid was neutralized and aq. Phase was clear, then washed with brine and the solvent removed under reduced pressure. A light yellow oil was isolated in $55 \%$ yield $(3.19 \mathrm{~g}, 10.4 \mathrm{mmol}) \cdot \mathrm{R}_{f}=0.39$ (cyclohexane: ethyl acetate, 2:1). ${ }^{1} \mathrm{H}$ NMR $\left(400 \mathrm{MHz}, \mathrm{CDCl}_{3}, \mathrm{ppm}\right), \delta=2.06-2.15(\mathrm{~m}, 2 \mathrm{H}), 2.74-2.83$ (m, $2 \mathrm{H}), 3.45$ - $3.54(\mathrm{~m}, 2 \mathrm{H}), 6.11-6.18(\mathrm{~m}, 1 \mathrm{H}), 6.91$ (bs., $1 \mathrm{H}) .{ }^{13} \mathrm{C} \mathrm{NMR}\left(100 \mathrm{MHz}, \mathrm{CDCl}_{3}, \mathrm{ppm}\right), \delta=$ 24.1, 25.5, 38.9, 93.3 (q, $J=36.5 \mathrm{~Hz}$ ), 115.7 (q, $J=288 \mathrm{~Hz}), 120.4(\mathrm{q}, J=281 \mathrm{~Hz}), 158.7$ (q, $J=37.4 \mathrm{~Hz})$, 163.4, 168.2. $\left.{ }^{19} \mathrm{~F} \mathrm{NMR} \mathrm{(471} \mathrm{MHz,} \mathrm{CDCl}_{3}, \mathrm{ppm}\right) \delta=-75.94,-78.86 . \mathrm{El}\left(\mathrm{m} / \mathrm{z}, 70 \mathrm{eV}, 70{ }^{\circ} \mathrm{C}\right): 306$ (5) [M] $]^{+}$, 288 (34), 262 (49), 200 (42), 193 (75), 167 (100), 140 (77), 126 (30), 98 (26). HRMS ( $\left.\mathrm{C}_{9} \mathrm{H}_{8} \mathrm{~N}_{2} \mathrm{O}_{3} \mathrm{~F}_{6}\right)\left[\mathrm{M}^{+}\right]$: calc. 306.0434 , found 306.0433 . IR (ATR, ṽ): 3316, 1802, 1740, 1650, 1557, 1447, 1370, 1311, 1272, $1149,1017,871,772,722,699,533,420 \mathrm{~cm}^{-1}$.

\section{Solid-supported oxazolones:}

Polystyrenemethyl-3-(4-methyl-5-oxo-2-(trifluoromethyl)-2,5-dihydrooxazol-2-yl)propanoate (3a).

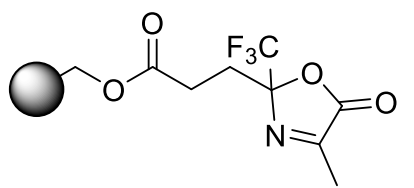

Acrylester resin 1 (batch 1.2, $1.10 \mathrm{~g}$, loading: $0.956 \mathrm{mmol} / \mathrm{g}, 1.05 \mathrm{mmol}$ ) was added to a solution of 4-methyl-2-(trifluoromethyl)oxazol-5(2H)-one (701 mg, $4.20 \mathrm{mmol}, 4.00$ equiv.) and $\mathrm{NEt}_{3}(0.439 \mathrm{~mL}, 3.15 \mathrm{mmol}, 3.00$ equiv.) in $50.0 \mathrm{~mL}$ of $\mathrm{CH}_{2} \mathrm{Cl}_{2}$ and shaken for $16 \mathrm{~h}$ at room temperature.

The resin was purified according to the general washing procedure. After drying under high vacuum, the white target resin was obtained in quantitative yield $(1.34 \mathrm{~g}$, loading: $0.745 \mathrm{mmol} / \mathrm{g}) .{ }^{13} \mathrm{C}$ GelNMR $\left(100 \mathrm{MHz}, \mathrm{CDCl}_{3}, \mathrm{ppm}\right), \delta=14.3,26.6,67.1,101.5,163.6,166.2,171.0 .{ }^{19} \mathrm{~F}$ Gel NMR $(376 \mathrm{MHz}$, $\left.\mathrm{CDCl}_{3}, \mathrm{ppm}\right)=-80.03 . \mathrm{IR}(\mathrm{ATR}, \tilde{\mathrm{v}}):$ 3024, 2921, 1807, 1736, 1600, 1492, 1451, 1270, 1193, 1067, 1026, $754,695,536 \mathrm{~cm}^{-1}$.

\begin{tabular}{cccccc} 
No & start.amount[g] & mmol & yield[g] & conversion & loading[mmol/g] \\
\hline 3a.1 [from 1.4] & 1.10 & 0.998 & 1.34 & quant & 0.745 \\
\hline
\end{tabular}

\section{Polystyrenemethyl-3-(4-(4-hydroxyphenyl)-5-oxo-2-(trifluoromethyl)-2,5-dihydrooxazol-2-yl)} propanoate (3b).

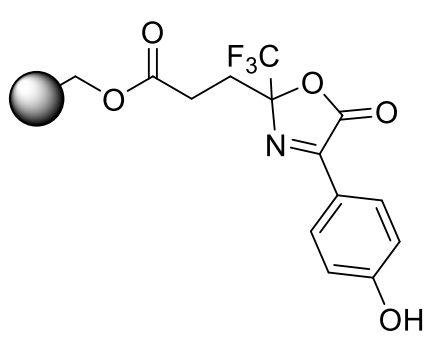

Acrylester resin 1 (batch 1.1, $2.55 \mathrm{~g}$, loading: $0.867 \mathrm{mmol} / \mathrm{g}, 2.21 \mathrm{mmol}$ ) was added to a solution of oxazolone $2 \mathrm{~b}(2.52 \mathrm{~g}, 10.3 \mathrm{mmol}$, 4.66 equiv.) and $\mathrm{NEt}_{3}(2.60 \mathrm{~g}, 3.59 \mathrm{~mL}, 25.7 \mathrm{mmol}, 11.6$ equiv.) in 15.0 $\mathrm{mL}$ of $\mathrm{CH}_{2} \mathrm{Cl}_{2}$ and shaken for $16 \mathrm{~h}$ at room temperature. The resin was purified according to the general washing procedure. After drying under high vacuum, the green target resin was obtained in quantitative yield ( $3.33 \mathrm{~g}$, loading: $0.664 \mathrm{mmol} / \mathrm{g})$. The reaction has been performed several times and the yields are summarized in the table given below. ${ }^{13} \mathrm{C} \mathrm{Gel-NMR}\left(100 \mathrm{MHz}, \mathrm{CDCl}_{3}\right.$, $\mathrm{ppm}), \delta=26.9,67.2,99.8-100.6,116.1,131.6,159.4,161.4,163.0,171.7 .{ }^{19} \mathrm{~F}$ Gel NMR $(376 \mathrm{MHz}$, 
$\left.\mathrm{CDCl}_{3}, \mathrm{ppm}\right)=-84.4 . \mathrm{IR}(\mathrm{ATR}, \tilde{\mathrm{v}}):$ 3024, 2922, 1799, 1737, 1599, 1514, 1492, 1450, 1142, 1068, 845, $753,695,534 \mathrm{~cm}^{-1}$.

\begin{tabular}{cccccc} 
No & start.amount[g] & mmol & yield[g] & conversion & loading[mmol/g] \\
\hline 3b.1 [from 1.1] & 2.55 & 2.21 & 3.33 & quant & 0.664 \\
3b.2 [from 1.2] & 3.65 & 3.49 & 4.95 & quant & 0.705 \\
3b.3 [from 1.3] & 4.69 & 4.48 & 6.91 & quant & 0.649 \\
3b.4 [from 1.4] & 1.50 & 1.36 & 1.96 & quant & 0.696 \\
3b.5 [from 1.3] & 10.1 & 0.96 & 14.2 & quant & 0.774 \\
\hline
\end{tabular}

Polystyrenemethyl-3-(4-phenyl-5-oxo-2-(trifluoromethyl)-2,5-dihydrooxazol-2-yl)propanoate (3c).<smiles>O=C(CCC(F)(F)F)N=C(OC(=O)O)c1ccccc1</smiles>

Acrylester resin 1 (batch 1.1, $2.00 \mathrm{~g}$, loading: $0.867 \mathrm{mmol} / \mathrm{g}, 1.73 \mathrm{mmol}$ ) was added to a solution of oxazolone $2 \mathrm{c}(1.75 \mathrm{~g}, 7.14 \mathrm{mmol}, 3.92$ equiv.) and $\mathrm{NEt}_{3}\left(1.80 \mathrm{~g}, 2.49 \mathrm{~mL}, 17.9 \mathrm{mmol}, 9.80\right.$ equiv.) in $20 \mathrm{~mL}$ of $\mathrm{CH}_{2} \mathrm{Cl}_{2}$ and shaken for $16 \mathrm{~h}$ at room temperature. The resin was purified according to the general washing procedure. After drying under high vacuum, the yellow target resin was obtained in quantitative yield $(2.45 \mathrm{~g}$, loading: $0.708 \mathrm{mmol} / \mathrm{g})$. The reaction has been performed twice and the yields are summarized in the table given below. ${ }^{13} \mathrm{C}$ Gel-NMR (100 MHz, $\left.\mathrm{CDCl}_{3}, \mathrm{ppm}\right), \delta=26.6,26.8,66.9,100.4$ (q, $J=29 \mathrm{~Hz}$ ), 125.6, 129.2, 134.0, 160.5, 162.4, 171.0. $\left.{ }^{19} \mathrm{~F} \mathrm{Gel} \mathrm{NMR} \mathrm{(376} \mathrm{MHz,} \mathrm{CDCl} 3, \mathrm{ppm}\right)=-79.7$. IR (ATR, ṽ): 2024, 2020, $1803,1737,1601,1492,1450,1152,1069,1027,750,695,527,383 \mathrm{~cm}^{-1}$.

\begin{tabular}{cccccc} 
No & start.amount[g] & Mmol & yield[g] & conversion & loading[mmol/g] \\
\hline 3c.1 [from 1.1] & 2.00 & 1.73 & 2.45 & quant & 0.708 \\
3c.2 [from 1.5] & 6.31 & 6.03 & 7.77 & quant & 0.784 \\
\hline
\end{tabular}

Polystyrenemethyl-3-(4-isobutyl-5-oxo-2-(trifluoromethyl)-2,5-dihydrooxazol-2-yl)propanoate (3d).<smiles>CC(C)CC1=NC(F)(CCC(=O)OCO)OC1=O</smiles>
Acrylester resin 1 (batch 1.1, $3.51 \mathrm{~g}$, loading: $0.867 \mathrm{mmol} / \mathrm{g}, 3.04 \mathrm{mmol}$ ) was added to a solution of oxazolone $2 \mathrm{~d}(2.00 \mathrm{~g}, 9.56 \mathrm{mmol}, 3.14$ equiv.) and DMAP (389 mg, $3.19 \mathrm{mmol}, 1.05$ equiv.) in $15.0 \mathrm{~mL}$ of $\mathrm{CH}_{2} \mathrm{Cl}_{2}$ and shaken for $16 \mathrm{~h}$ at room temperature. The resin was purified according to the general washing procedure. After drying under high vacuum, the off white target resin was obtained in quantitative yield $(4.36 \mathrm{~g}$, loading: $0.697 \mathrm{mmol} / \mathrm{g})$. The reaction has been performed several times and the yields are summarized in the table given below. ${ }^{13} \mathrm{C}$ GelNMR (100 MHz, CDCl $3, \mathrm{ppm}), \delta=22.2,22.4,24.5,26.1,26.6,36.7,66.8,101.4$ (q, $J=29 \mathrm{~Hz}$ ), 163.5, 168.5. ${ }^{19} \mathrm{~F}$ Gel NMR $\left(376 \mathrm{MHz}, \mathrm{CDCl}_{3}, \mathrm{ppm}\right)=-79.9$. IR (ATR, ṽ): 3024, 2920, 1806, 1735, 1601, 1492, $1451,1192,1073,1027,755,695,536 \mathrm{~cm}^{-1}$.

\begin{tabular}{cccccc} 
No & start.amount[g] & mmol & yield[g] & conversion & loading[mmol/g] \\
\hline 3d.1 [from 1.1] & 3.51 & 3.04 & 4.36 & quant & 0.697 \\
3d.2 [from 1.1] & 3.29 & 3.15 & 3.99 & quant & 0.789 \\
3d.3 [from 1.6] & 3.52 & 3.36 & 4.46 & quant & 0.797 \\
\hline
\end{tabular}




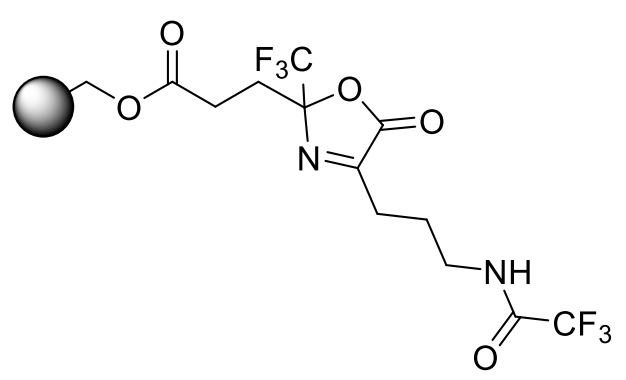

Acrylester resin 1 (batch 1.2, $1.40 \mathrm{~g}$, loading: $0.956 \mathrm{mmol} / \mathrm{g}$, $1.34 \mathrm{mmol}$ ) was added to a solution of 2,2,2-trifluoro- $\mathrm{N}$-(3-

(5-oxo-2-(trifluoromethyl)-2,5-dihydrooxazol-4-yl)propyl) acetamide $(1.56 \mathrm{~g}, 5.11 \mathrm{mmol})$ and triethylamine (0.534 mL, $3.83 \mathrm{mmol}$ ) in $50.0 \mathrm{~mL}$ of $\mathrm{CH}_{2} \mathrm{Cl}_{2}$ and shaken for $16 \mathrm{~h}$ at room temperature. The resin was purified according to the general washing procedure. After drying under high vacuum, the white target resin was obtained in quantitative yield (1.84 g, loading: $0.726 \mathrm{mmol} / \mathrm{g})$. The reaction has been performed several times and the yields are summarized in the table given below. ${ }^{13} \mathrm{C} \mathrm{Gel-NMR}\left(100 \mathrm{MHz}, \mathrm{CDCl}_{3}, \mathrm{ppm}\right), \delta=24.2,25.3,26.5$, 39.7, 49.6, 66.9, 106.4, 125.7, 140.0, 157.0, 168.3. $\left.{ }^{19} \mathrm{~F} \mathrm{NMR} \mathrm{(376} \mathrm{MHz,} \mathrm{CDCl}_{3}, \mathrm{ppm}\right) \delta=-80.5,-84.8$. IR (ATR, ṽ): 3025, 2921, 2850, 1807, 1719, 1648, 1556, 1492, 1451, 1152, 1027, 755, 696, $537 \mathrm{~cm}^{-1}$.

\begin{tabular}{cccccc} 
No & start.amount[g] & mmol & yield[g] & conversion & loading[mmol/g] \\
\hline 3e.1 [from 1.2] & 0.70 & 0.669 & 0.945 & quant & 0.709 \\
3e.2 [from 1.2] & 1.40 & 1.39 & 1.84 & quant & 0.726 \\
\hline
\end{tabular}

\section{Synthesis and Characterization of Single Compounds}

\section{3-(4-Hydroxyphenyl)quinoxalin-2(1H)-one (5ba).}

Resin 3b (batch 3b.5, $2.00 \mathrm{~g}, 0.7743 \mathrm{~mol} / \mathrm{g}$ ) was swollen in methanol (10.0 ml), benzene-1,2-<smiles>O=c1[nH]c2ccccc2nc1-c1ccc(O)cc1</smilesdiamine $(0.670 \mathrm{~g}, 6.20 \mathrm{mmol}, 4.00$ equiv.) was added and the reaction mixture was stirred at $80{ }^{\circ} \mathrm{C}$ for 2 days. The reaction mixture was then cooled to $4{ }^{\circ} \mathrm{C}$, filtered through a syringe phase separator and washed with $5 \mathrm{~mL}$ of cold methanol. The yellow precipitate, mixed with resin, was then dissolved in hot THF/ methanol 1:1 and separated from the resin in a syringe phase separator and the solvent of the filtrate removed under reduced pressure. The crude product was recrystallized from methanol to yield $248 \mathrm{mg}$ of the yellow solid product in $67 \%$ yield. $\mathrm{R}_{f}$ (dichloromethane/methanol, $\left.20: 1\right)=0.33 .{ }^{1} \mathrm{H}\left(500 \mathrm{MHz}\right.$, DMSO- $d_{6}, \mathrm{ppm}$ ) $\delta=12.46(\mathrm{~s}, 1 \mathrm{H}), 9.98(\mathrm{~s}, 1 \mathrm{H}), 8.32(\mathrm{~d}, J=8.8 \mathrm{~Hz}, 2 \mathrm{H}), 7.78(\mathrm{~d}, J=8.0 \mathrm{~Hz}, 1 \mathrm{H}), 7.48(\mathrm{t}, J=8.3 \mathrm{~Hz}, 1$ H), $7.29(\mathrm{t}, J=8.9 \mathrm{~Hz}, 2 \mathrm{H}), 6.86(\mathrm{~d}, J=8.9 \mathrm{~Hz}, 2 \mathrm{H}) .{ }^{13} \mathrm{C}$ NMR $\left(125 \mathrm{MHz}\right.$, DMSO- $\left.d_{6}, \mathrm{ppm}\right) \delta=159.7$, 154.7, 153.2, 132.1, 131.7, 131.2, 129.5, 128.3, 126.7, 123.3, 114.9, 114.8. IR (ATR): $\tilde{v}=2848,1660$, $1607,1536,1516,1478,1430,1361,1297,1279,1213,1173,1032,888,856,837,794,756,744$, 656, 642, 618, 588, 534, 519, $467 \mathrm{~cm}^{-1}$. El (m/z, $\left.70 \mathrm{eV}, 220^{\circ} \mathrm{C}\right): 239$ [M+- H], 238 [M+] (67), 211 (15), 210 (100), 182 (17), 181 (23). HRMS $\left(\mathrm{C}_{14} \mathrm{H}_{10} \mathrm{O}_{2} \mathrm{~N}_{2}\right)$ : calc.: 238.0737; found: 238.0738. EA calc.: C: 70.58, $\mathrm{H}: 4.23, \mathrm{~N}: 11.76$; found: C: 69.90, H: 4.31, N: 11.57 .

\section{3-Phenylquinoxalin-2(1H)-one (5ca).}<smiles>O=c1[nH]c2ccccc2nc1-c1ccccc1</smiles>

Resin 3c (batch 3c.2, $1.00 \mathrm{~g}, 0.7841 \mathrm{~mol} / \mathrm{g}$ ) was swollen in methanol $(7.0 \mathrm{ml})$, benzene-1,2-diamine ( $254 \mathrm{mg}, 2.35 \mathrm{mmol}, 3.00$ equiv.) was added and the reaction mixture was shaken at $80^{\circ} \mathrm{C}$ for 20 days under nitrogen atmosphere. The suspension was filtered through a syringe phase separator, the resin washed with methanol and THF and the solvent of the filtrate removed under reduced pressure. The crude product was purified by flash chromatography ( 4:1 $>>1: 4, \mathrm{CH}: \mathrm{EA})$ and 
recrystallized from ethanol to yield $63.0 \mathrm{mg}$ of a yellow solid in $36 \%$ yield, the product has been obtained via the same procedure via HPLC purification in $44 \%$ yield. $\mathrm{R}_{f}$ (cyclohexane/ethyl acetate, $1: 1)=0.26 .{ }^{1} \mathrm{H}$ NMR $\left(500 \mathrm{MHz}, \mathrm{DMSO}-d_{6}, \mathrm{ppm}\right) \delta=12.58(\mathrm{~s}, 1 \mathrm{H}), 8.30(\mathrm{dd}, J=7.8 \mathrm{~Hz}, J=1.8 \mathrm{~Hz}, 2 \mathrm{H})$, $7.84(\mathrm{~d}, J=8.0 \mathrm{~Hz}, 1 \mathrm{H}), 7.59-7.43(\mathrm{~m}, 4 \mathrm{H}), 7.33(\mathrm{td}, J=8.4 \mathrm{~Hz}, J=1.9 \mathrm{~Hz}, 2 \mathrm{H}) .{ }^{13} \mathrm{C}$ NMR $(126 \mathrm{MHz}$, DMSO- $d_{6}$, ppm) $\delta=154.6,154.1,135.6,132.1,132.0,130.3,130.2,129.2,128.8,127.9,123.4,115.1$. $\mathrm{EI}\left(\mathrm{m} / \mathrm{z}, 70 \mathrm{eV}, 130{ }^{\circ} \mathrm{C}\right): 222$ [M] ${ }^{+}$(89), 194 (100), 166 (3). HRMS ( $\left.\mathrm{C}_{14} \mathrm{H}_{10} \mathrm{ON}\right)$ : calc.: 222.0788; found: 222.0787. IR (ATR, v): 1657, 1531, 1475, 1429, 1283, 1212, 1188, 1145, 1006, 947, 906, 806, 764, 732, $687,631,589,552,525,469,423$. EA: calc.: C: $75.66, \mathrm{H}: 4.54, \mathrm{~N}: 12.60$; found: C: $75.47, \mathrm{H}: 4.53, \mathrm{~N}$ : 12.54 .

\section{6-Methyl-3-phenylquinoxalin-2(1H)-one (5cb).}<smiles>Cc1cc2nc(-c3ccccc3)c(=O)[nH]c2cc1C</smiles>

Resin 3c (batch 3c.2, $1.00 \mathrm{~g}, 0.7841 \mathrm{~mol} / \mathrm{g}$ ) was swollen in methanol (7.0 $\mathrm{mL}$ ), 4-methylbenzene-1,2-diamine ( $287 \mathrm{mg}, 2.35 \mathrm{mmol}, 3.00$ equiv.) was added and the reaction mixture was shaken at $80{ }^{\circ} \mathrm{C}$ for 20 days under nitrogen atmosphere. The suspension was filtered through a syringe phase separator, the resin washed with methanol and THF and the solvent of the filtrate was removed under reduced pressure. The crude product was purified by flash chromatography (silica, 1:4 -> 1:1, CH:EA) and recrystallized from ethanol to yield $102 \mathrm{mg}$ of a yellow solid as an inseparable mixture of isomers in $55 \%$ yield. $R_{f}$ (dichloromethane/methanol, 30:1) $=0.30$. ${ }^{1} \mathrm{H}$ NMR $\left(500 \mathrm{MHz}, \mathrm{DMSO}-d_{6}, \mathrm{ppm}\right) \delta=12.51(\mathrm{~s}, 1 \mathrm{H}), 8.34-8.26(\mathrm{~m}, 2 \mathrm{H}), 7.71(\mathrm{~d}, J=8.2 \mathrm{~Hz}, 0.5 \mathrm{H})$, $7.64(\mathrm{~s}, 0.5 \mathrm{H}), 7.54-7.44(\mathrm{~m}, 3 \mathrm{H}), 7.37(\mathrm{~d}, J=6.8 \mathrm{~Hz}, 0.5 \mathrm{H}), 7.23(\mathrm{~d}, J=8.3 \mathrm{~Hz}, 0.5 \mathrm{H}), 7.14(\mathrm{~d}, J=$ $8.2 \mathrm{~Hz}, 0.5 \mathrm{H}), 7.11(\mathrm{~s}, 0.5 \mathrm{H}), 2.42(\mathrm{~s}, 1.5 \mathrm{H}), 2.39(\mathrm{~s}, 1.5 \mathrm{H}) .{ }^{13} \mathrm{C}$ NMR $\left(125 \mathrm{MHz}, \mathrm{DMSO}-d_{6}, \mathrm{ppm}\right) \delta=$ 154.7, 154.5, 153.9, 152.9, 140.6, 135.7, 135.7, 132.7, 132.0, 131.6, 130.3, 130.1, 130.0, 129.8, 129.2, $129.1,128.5,128.3,127.8,124.8,114.8,114.7,21.4,20.4$. El (m/z, $\left.70 \mathrm{eV}, 140{ }^{\circ} \mathrm{C}\right): 236[\mathrm{M}]^{+}(100)$, 208 (87), 194 (5). HRMS ( $\mathrm{C}_{15} \mathrm{H}_{12} \mathrm{ON}$ ): calc.: 236.0944; found: 236.0943. IR (ATR, ṽ): 1655, 1485, 1443, 1410, 1269, 1243, 1186, 1010, 879, 812, 800, 758, 730, 687, 627, 593, 577, 555, 527, 458, 435, 393. EA: calc.: C: $76.25, \mathrm{H}: 5.12, \mathrm{~N}: 11.86$; found: C: $76.03, \mathrm{H}: 5.13, \mathrm{~N}: 11.82$.

\section{3-Isobutylquinoxalin-2(1H)-one (5da).}

According to general procedure $3,300 \mathrm{mg}$ of resin $\mathbf{3 d}$ (batch $3 \mathrm{~d} .2$,<smiles>CC(C)Cc1nc2ccccc2[nH]c1=O</smiles>
$0.789 \mathrm{mmol} / \mathrm{g}, 0.237 \mathrm{mmol}$ ) were converted with $74.0 \mathrm{mg}$ of benzene-1,2diamine (0.687 mmol, 3.01 equiv.) in $4.0 \mathrm{~mL}$ of methanol to give $40.5 \mathrm{mg}$ ( $0.200 \mathrm{mmol}$ ) of a yellow solid in $84 \%$ yield. $\mathrm{R}_{f}$ (cyclohexane/ethyl acetate, $2: 1$ ) $=0.30 .{ }^{1} \mathrm{H} \mathrm{NMR}(400 \mathrm{MHz}, \mathrm{MeOD}, \mathrm{ppm}), \delta=0.97(\mathrm{~d}, J=6.6 \mathrm{~Hz}, 6 \mathrm{H}), 2.27(\mathrm{~m}, 1 \mathrm{H}), 2.75(\mathrm{~d}, J=7.3 \mathrm{~Hz}$, $2 \mathrm{H}), 7.21-7.32(\mathrm{~m}, 2 \mathrm{H}), 7.40-7.48(\mathrm{~m}, 1 \mathrm{H}), 7.76(\mathrm{~d}, J=8.1 \mathrm{~Hz}, 1 \mathrm{H}) .{ }^{13} \mathrm{C}$ NMR $\left(100 \mathrm{MHz}, \mathrm{CDCl}_{3}\right.$, ppm), $\delta=22.0,26.7,41.7,114.9,123.5,127.7,129.6,131.0,131.9,155.5,161.2$. El (m/z, $70 \mathrm{eV}, 100$ $\left.{ }^{\circ} \mathrm{C}\right):$ 202/203 (33/23) [M] ${ }^{+}, 187 / 188$ (32/22), 160/161 (100/66), 131/132 (43/22), 104 (24). HRMS $\left(\mathrm{C}_{12} \mathrm{H}_{14} \mathrm{NO}\right)\left[\mathrm{M}^{+}\right]$: calc. 202.1101, found 202.1102. IR (ATR, ṽ): 2951, 2865, 2161, 1660, 1636, 1599, $1584,1556,1487,1465,1429,1383,1365,1325,1174,1025,957,903,827,755,720,664,599,577$, $536,480,468,414,390 \mathrm{~cm}^{-1}$.

\section{Mixture of 3-isobutyl-6-methylquinoxalin-2(1H)-one and 3-isobutyl-7-methylquinoxalin-2(1H)-one} (5db).<smiles>Cc1cc2nc(CC(C)C)c(=O)[nH]c2cc1C</smiles>

According to general procedure $3,300 \mathrm{mg}$ of resin $\mathbf{3 d}$ (batch $3 \mathrm{~d} .2$, $0.789 \mathrm{mmol} / \mathrm{g}, 0.237 \mathrm{mmol}$ ) were converted with $84.0 \mathrm{mg}$ of 4-methyl benzene-1,2-diamine ( $0.687 \mathrm{mmol}, 2.90$ equiv.) in $4.0 \mathrm{~mL}$ of methanol to give $33.4 \mathrm{mg}(0.154 \mathrm{mmol}, 216.3 \mathrm{~g} / \mathrm{mol})$ of a white solid as inseparable mixture of isomers in $65 \%$ 
yield. $\mathrm{R}_{f}$ (cyclohexane/ethyl acetate, $\left.2: 1\right)=0.29 .{ }^{1} \mathrm{H}$ NMR (500 MHz, DMSO, ppm), $\delta=0.92(2 \mathrm{dd}, J=$ $6.7 \mathrm{~Hz}, 6 \mathrm{H}), 2.18-2.24(\mathrm{~m}, 1 \mathrm{H}), 2.62-2.65(\mathrm{~m}, 2 \mathrm{H}), 7.05(\mathrm{~s}, 0.5 \mathrm{H}), 7.07(\mathrm{~d}, J=8.2 \mathrm{~Hz}, 0.5 \mathrm{H}), 7.16$ (d, $J=8.2 \mathrm{~Hz}, 0.5 \mathrm{H}), 7.29(\mathrm{dd}, J=8.2 \mathrm{~Hz}, J=1.2 \mathrm{~Hz}, 0.5 \mathrm{H}), 7.52(\mathrm{~s}, 0.5 \mathrm{H}), 7.59(\mathrm{~d}, J=8.2 \mathrm{~Hz}, 0.5 \mathrm{H}), 12.2$ (bs, $0.5 \mathrm{H}) .{ }^{13} \mathrm{C}$ NMR $\left(125 \mathrm{MHz}, \mathrm{CDCl}_{3}, \mathrm{ppm}\right), \delta=20.4,21.2,22.5,22.6,26.1,26.2,41.4,41.5,114.8$, $114.9,124.4,127.8,127.9,129.4,129.9,130.5,131.6,131.6,132.3,139.5,154.7,154.9,160.0,161.1$. EI (m/z, $70 \mathrm{eV}):$ 216/217 (37/22) [M] ${ }^{+}, 201 / 202$ (35/22), 174/175 (100/55), 145/146 (33/12), 119 (7). HRMS $\left(\mathrm{C}_{13} \mathrm{H}_{16} \mathrm{~N}_{2} \mathrm{O}\right)[\mathrm{M}]^{+}$: calc. 216.1257, found 216.1258. IR (ATR, ṽ): 2953, 2865, 2218, 1637, 1608, $1583,1500,1460,1385,1364,1164,1126,901,868,820,782,726,685,655,601,570,506,478$, $461,438,421,391 \mathrm{~cm}^{-1}$.

\section{2-Phenylpyrido[3,4-b]pyrazin-3(4H)-one (5cc)}<smiles>O=c1[nH]c2ccncc2nc1-c1ccccc1</smiles>

Pyridin-3,4-diamine (103 mg, $0.941 \mathrm{~mol}, 1.20$ equiv.) was dissolved in THF $(6.00 \mathrm{~mL})$ and triethylamine ( $4.37 \mu \mathrm{l}, 0.031 \mathrm{mmol}, 0.04$ equiv.) was added. The solution was stirred for $5 \mathrm{~min}$, then the resin $\mathbf{3 c}$ (batch $3 c .2,1.00 \mathrm{~g}, 0.784 \mathrm{~mol} / \mathrm{g}$ ) was added and the reaction mixture was shaken at $80{ }^{\circ} \mathrm{C}$ for 13 days. The suspension was filtered through a syringe phase separator, the resin washed with methanol and the solvent of the filtrate removed under reduced pressure. The crude product was dissolved in $0.5 \mathrm{~N}$ $\mathrm{HCl}$, neutralized with $\mathrm{NaHCO}_{3}$, extracted with $\mathrm{EA}$ and the solvent removed under reduced pressure. The crude product was purified by flash chromatography as an inseparable mixture of isomers $(20: 1$, DCM:methanol) to yield $51.2 \mathrm{mg}$ of a yellow solid in $73 \%$ yield (Isomer 1 : Isomer $2=1.38: 1.00) . \mathrm{R}_{f}$ (dichloromethane/methanol, 10:1) $=0.49$. Isomer $1 .{ }^{1} \mathrm{H}$ NMR $\left(500 \mathrm{MHz}\right.$, DMSO-d $\left.d_{6}, \mathrm{ppm}\right) \delta=12.77(\mathrm{~s}$, $1 \mathrm{H}), 8.98(\mathrm{~s}, 1 \mathrm{H}), 8.49(\mathrm{~d}, J=5.5 \mathrm{~Hz}, 1 \mathrm{H}), 8.29(\mathrm{dd}, J=19.6 \mathrm{~Hz}, J=7.3 \mathrm{~Hz}, 2 \mathrm{H}), 7.54(\mathrm{dt}, J=18.6 \mathrm{~Hz}, J$ $=7.0 \mathrm{~Hz}, 3 \mathrm{H}), 7.24(\mathrm{~d}, J=5.4 \mathrm{~Hz}, 1 \mathrm{H}) .{ }^{13} \mathrm{C}$ NMR $\left(250 \mathrm{MHz}, \mathrm{DMSO}-d_{6}, \mathrm{ppm}\right) \delta=159.9,154.9,150.3$, $148.8,137.4,135.1,130.6,129.3,128.6,127.9,121.3$. Isomer 2. ${ }^{1} \mathrm{H}$ NMR (500 MHz, DMSO-d6, ppm) $\delta=12.77(\mathrm{~s}, 1 \mathrm{H}), 8.68(\mathrm{~s}, 1 \mathrm{H}), 8.46(\mathrm{~d}, J=5.3 \mathrm{~Hz}, 1 \mathrm{H}), 8.29(\mathrm{dd}, J=19.6 \mathrm{~Hz}, J=7.3 \mathrm{~Hz}, 2 \mathrm{H}), 7.77(\mathrm{~d}, J$ $=5.2 \mathrm{~Hz}, 1 \mathrm{H}), 7.54(\mathrm{dt}, J=18.6 \mathrm{~Hz}, J=7.0 \mathrm{~Hz}, 3 \mathrm{H}) .{ }^{13} \mathrm{C}$ NMR $\left(250 \mathrm{MHz}, \mathrm{DMSO}-d_{6}, \mathrm{ppm}\right) \delta=175.9$, $156.2,154.3,143.5,138.5,135.6,135.0,131.1,129.7,128.0,109.5$. Isomers 1+2. IR (ATR): $\tilde{v}=2696$, $1667,1595,1537,1479,1445,1416,1335,1258,1207,1173,1043,1005,908,842,809,766,740$, 684, 630, 599, 551, 524, 475, $416 \mathrm{~cm}^{-1}$. El (m/z, $\left.70 \mathrm{eV}, 130{ }^{\circ} \mathrm{C}\right): 223$ [M+] (100), 195 (86), 69 (20). HRMS $\left(\mathrm{C}_{13} \mathrm{H}_{9} \mathrm{O}_{1} \mathrm{~N}_{3}\right)$ : calc.: 223.0740; found: 223.0738 .

\section{3-Methyl-4a,5,6,7,8,8a-hexahydroquinoxalin-2(1H)-one (5ad).}<smiles>CC1=NC2CCCCC2NC1=O</smiles>

According to general procedure 3, $300 \mathrm{mg}$ of resin $3 \mathrm{a}$ (batch $3 a .1,0.745 \mathrm{mmol} / \mathrm{g}$, $0.224 \mathrm{mmol})$ were converted with $85.0 \mathrm{mg}$ of cyclohexane-1,2-diamine $(0.740$ mmol, 3.30 equiv.) in $4.0 \mathrm{~mL}$ of methanol to give $19 \mathrm{mg}(0.114 \mathrm{mmol})$ of the target substance in $51 \%$ yield as a beige solid. $\mathrm{R}_{f}$ (dichloromethane/ methanol, 10:1) = 0.39. ${ }^{1} \mathrm{H}$ NMR $\left(300 \mathrm{MHz}, \mathrm{CDCl}_{3}, \mathrm{ppm}\right), \delta=1.23-1.45(\mathrm{~m}, 4 \mathrm{H}), 1.74-1.99(\mathrm{~m}, 3 \mathrm{H}), 2.21(\mathrm{~d}, J=2.5 \mathrm{~Hz}$, $3 \mathrm{H}), 2.29$ (d, J = 6.2 Hz, $1 \mathrm{H}), 2.97-3.20$ (m, $2 \mathrm{H}), 6.70$ (bs., $1 \mathrm{H}) .{ }^{13} \mathrm{C} \mathrm{NMR}\left(75 \mathrm{MHz}, \mathrm{CDCl}_{3}, \mathrm{ppm}\right), \delta=$ 20.8, 23.6, 25.2, 31.0, 31.7, 54.4, 62.4, 158.9, 162.9. El (m/z, $\left.70 \mathrm{eV}, 80^{\circ} \mathrm{C}\right): 166$ (100) [M] ${ }^{+}, 137$ (8), 95 (37). HRMS $\left(\mathrm{C}_{9} \mathrm{H}_{14} \mathrm{~N}_{2} \mathrm{O}\right)[\mathrm{M}]^{+}$: calc. 166.1101 found 166.1102. IR (ATR, ṽ): 3220, 2927, 2855, 1681, $1622,1449,1357,1315,1202,1117,875,770,730,685,572,495,477,420,395 \mathrm{~cm}^{-1}$. 
3-(4-Hydroxyphenyl)-4a,5,6,7,8,8a-hexahydroquinoxalin-2(1H)-one (5bd).

According to general procedure $3,300 \mathrm{mg}$ of resin $3 \mathbf{b}$ (batch $3 \mathrm{~b} .4,0.696 \mathrm{mmol} / \mathrm{g}, 0.209 \mathrm{mmol}$ ) were<smiles>O=C1NC2CCCCC2N=C1c1ccc(O)cc1</smiles>
converted with $76.0 \mathrm{mg}$ of cyclohexane-1,2-diamine $(0.669 \mathrm{mmol}, 3.2$ equiv.) in $4.0 \mathrm{~mL}$ of methanol to give $46.2 \mathrm{mg}(0.189 \mathrm{mmol})$ of the target substance in $90 \%$ yield as a dark yellow solid. $\mathrm{R}_{f}$ (dichloromethane/ methanol, 10:1) $=0.32 .{ }^{1} \mathrm{H}$ NMR $(250 \mathrm{MHz}$, MeOD, ppm), $\delta=1.32-1.55$ $(\mathrm{m}, 4 \mathrm{H}), 1.77-1.91(\mathrm{~m}, 2 \mathrm{H}), 1.99-2.09(\mathrm{~m}, 1 \mathrm{H}), 2.35(\mathrm{~d}, J=5.8 \mathrm{~Hz}, 1 \mathrm{H})$, $3.14(\mathrm{dd}, J=6.3 \mathrm{~Hz}, J=2.6 \mathrm{~Hz}, 2 \mathrm{H}), 6.74-6.84(\mathrm{~m}, 2 \mathrm{H}), 7.65-7.74(\mathrm{~m}, 2 \mathrm{H}) .{ }^{13} \mathrm{C} \mathrm{NMR}\left(63 \mathrm{MHz}, \mathrm{CDCl}_{3}\right.$ +MeOD, ppm), $\delta=23.4,24.8,30.3,31.4,53.6,62.3,114.6,126.2,130.4,158.6,159.1,161.2$. El (m/z, $\left.70 \mathrm{eV}, 130{ }^{\circ} \mathrm{C}\right): 244(100)[\mathrm{M}]^{+}, 215(20), 179(74), 173(57), 120(90), 69(53) . \mathrm{HRMS}\left(\mathrm{C}_{14} \mathrm{H}_{16} \mathrm{NO}_{2}\right)[\mathrm{M}]^{+}:$ calc. 244.1206, found 244.1207. IR (ATR, ṽ): 3252, 2932, 2859, 2349, 1670, 1585, 1512, 1447, 1364, 1273, 1239, 1171, 1147, 1111, 1066, 985, 869, 840, 797, 743, 719, 650, 626, 573, 515, 483, 457, 411 $\mathrm{cm}^{-1}$. EA $\left(\mathrm{C}_{14} \mathrm{H}_{16} \mathrm{~N}_{2} \mathrm{O}_{2}\right)$ : calc. C 68.83, H 6.60, N 11.47; found. C 68.30, H 6.64, N 11.27.

\section{3-Phenyl-4a,5,6,7,8,8a-hexahydroquinoxalin-2(1H)-one (5cd).}<smiles>O=C1NC2CCCCC2N=C1c1ccccc1</smiles>

According to general procedure 3, $300 \mathrm{mg}$ of resin 3c (batch 3c.1, 0.708 $\mathrm{mmol} / \mathrm{g}, 0.212 \mathrm{mmol}$ ) were converted with $76.0 \mathrm{mg}$ of cyclohexane-1,2diamine ( $0.669 \mathrm{mmol}, 3.16$ equiv.) in $4.0 \mathrm{~mL}$ of methanol to give $41.7 \mathrm{mg}$ $(0.183 \mathrm{mmol})$ of the target substance in $86 \%$ yield as a light brown solid. $\mathrm{R}_{f}$ (cyclohexane/ethyl acetate, $1: 1)=0.42 .{ }^{1} \mathrm{H}$ NMR $\left(250 \mathrm{MHz}^{\mathrm{C}} \mathrm{CDCl}_{3}, \mathrm{ppm}\right), \delta=$ 1.22 - $1.58(\mathrm{~m}, 4 \mathrm{H}), 1.78-2.04(\mathrm{~m}, 3 \mathrm{H}), 2.41-2.51(\mathrm{~m}, 1 \mathrm{H}), 3.24(\mathrm{dd}, J=6.6 \mathrm{~Hz}, J=2.6 \mathrm{~Hz}, 2 \mathrm{H}), 6.14$ (bs, $1 \mathrm{H}), 7.36-7.49$ (m, $3 \mathrm{H}), 7.86-7.95$ (m, $2 \mathrm{H}) .{ }^{13} \mathrm{C}$ NMR (63 MHz, MeOD, ppm), $\delta=25.0,26.4$, $31.7,33.0,55.3,64.3,129.1,130.2,131.5,136.8,159.9,164.0$. El (m/z, $\left.70 \mathrm{eV}, 90^{\circ} \mathrm{C}\right): 228(100)[\mathrm{M}]^{+}$, 199 (23), 157 (57), 145 (19), 104 (81). HRMS $\left(\mathrm{C}_{14} \mathrm{H}_{16} \mathrm{~N}_{2} \mathrm{O}_{3}\right)$ [M] : calc. 228.1257, found 228.1258. IR (ATR, ṽ): 3184, 3062, 2932, 2859, 2333, 1665, 1592, 1572, 1492, 1445, 1418, 1358, 1315, 1285, 1274, $1258,1200,1179,1145,1083,1064,1033,985,945,923,867,845,806,761,730,687,632,619$, $591,577,534,504,487,426,406 \mathrm{~cm}^{-1}$. EA $\left(\mathrm{C}_{14} \mathrm{H}_{16} \mathrm{~N}_{2} \mathrm{O}\right)$ : calc. C 73.66, H 7.06, N 12.27; found. C 73.04, H 7.07, N 12.06 .

\section{3-Isobutyl-4a,5,6,7,8,8a-hexahydroquinoxalin-2(1H)-one (5dd).}<smiles>CC(C)CC1=NC2CCCCC2NC1=O</smiles>

According to general procedure 3, $300 \mathrm{mg}$ of resin 3d (batch 3d.1, 0.697 $\mathrm{mmol} / \mathrm{g}, 0.209 \mathrm{mmol}$ ) were converted with $78.0 \mathrm{mg}$ of cyclohexane-1,2diamine (0.687 mmol, 3.29 equiv.) in $4.0 \mathrm{~mL}$ of methanol to give $24.8 \mathrm{mg}$ $(0.119 \mathrm{mmol})$ of the target substance in $57 \%$ yield as a orange solid. $\mathrm{R}_{f}$ (cyclohexane/ethyl acetate, $1: 1)=0.32 .{ }^{1} \mathrm{H}$ NMR $\left(300 \mathrm{MHz}, \mathrm{CDCl}_{3}, \mathrm{ppm}\right), \delta=0.93(2 \mathrm{xd}, J=6.8 \mathrm{~Hz}, 6$ H), 1.23 - $1.53(\mathrm{~m}, 4 \mathrm{H}), 1.75-1.96(\mathrm{~m}, 3 \mathrm{H}), 2.06(\mathrm{tt}, J=13.7 \mathrm{~Hz}, J=6.7 \mathrm{~Hz}, 1 \mathrm{H}), 2.18-2.37(\mathrm{~m}, 2 \mathrm{H})$, $2.70(\mathrm{dd}, J=13.2, J=6.4 \mathrm{~Hz}, 1 \mathrm{H}), 2.93-3.19(\mathrm{~m}, 2 \mathrm{H}), 6.46$ (bs, $1 \mathrm{H}) .{ }^{13} \mathrm{C} \mathrm{NMR}\left(75 \mathrm{MHz}, \mathrm{CDCl}_{3}, \mathrm{ppm}\right)$, $\delta=22.2,22.7,23.7,25.2,26.6,31.0,31.8,42.2,54.4,62.5,158.7,165.5 . \mathrm{El}\left(\mathrm{m} / \mathrm{z}, 70 \mathrm{eV}, 70{ }^{\circ} \mathrm{C}\right): 208$ $[\mathrm{M}]^{+}(16), 193$ (7), 166 (100), 138 (14). HRMS $\left(\mathrm{C}_{12} \mathrm{H}_{20} \mathrm{ON}_{2}\right)$ : calc. 208.1570, found 208.1568. IR (ATR, v): 3180, 3065, 2928, 2859, 1683, 1617, 1453, 1359, 1313, 1239, 1175, 1141, 1090, 1048, 798, 711, 608 , $574,533,511,478,419,401 \mathrm{~cm}^{-1}$. EA $\left(\mathrm{C}_{12} \mathrm{H}_{20} \mathrm{~N}_{2} \mathrm{O}\right)$ : calc. C 69.19, H 9.68, N 13.45; found. C 68.82, $\mathrm{H}$ 9.50, N 13.14 .

\section{3-(3-Aminopropyl)-4a,5,6,7,8,8a-hexahydroquinoxalin-2(1H)-one (5ed).}<smiles>O=C1NC2CCCCC2N=C1CCCNC(=O)C(F)(F)F</smiles>

According to general procedure 3, $300 \mathrm{mg}$ of resin 3e (batch 3e.1, $0.709 \mathrm{mmol} / \mathrm{g}, 0.213 \mathrm{mmol}$ ) were converted with $73.0 \mathrm{mg}$ of cyclohexane-1,2-diamine $(0.640 \mathrm{mmol}, 3.00$ equiv. $)$ in $4.0 \mathrm{~mL}$ of 
methanol to give $35.0 \mathrm{mg}(0.114 \mathrm{mmol})$ of the target substance in $54 \%$ yield as a white solid. $\mathrm{R}_{f}$ (ethyl acetate $)=0.40 .{ }^{1} \mathrm{H}$ NMR $\left(400 \mathrm{MHz} \mathrm{CDCl}_{3}, \mathrm{ppm}\right), \delta=1.13-1.48(\mathrm{~m}, 5 \mathrm{H}), 1.74-2.02(\mathrm{~m}, 5 \mathrm{H}), 2.55-$ $2.72(\mathrm{~m}, 1 \mathrm{H}), 2.76-2.86(\mathrm{~m}, 1 \mathrm{H}), 3.00-3.18(\mathrm{~m}, 2 \mathrm{H}), 3.30-3.44(\mathrm{~m}, 2 \mathrm{H}), 7.00$ (bs, $1 \mathrm{H}), 8.02$ (bs, 1 H). ${ }^{13} \mathrm{C}$ NMR $\left(100 \mathrm{MHz}, \mathrm{CDCl}_{3}, \mathrm{ppm}\right), \delta=23.5,24.3,25.1,30.6,30.8,31.4,39.8,54.2,62.4,115.9$ (q, ${ }^{1} \mathrm{~J}$ $=301 \mathrm{~Hz}), 157.1\left(\mathrm{q},{ }^{2} J=36.7 \mathrm{~Hz}\right), 158.7,165.3 .{ }^{19} \mathrm{~F} \mathrm{NMR}\left(376 \mathrm{MHz}, \mathrm{CDCl}_{3}, \mathrm{ppm}\right) \delta=-75.73 . \mathrm{El}(\mathrm{m} / \mathrm{z}, 70$ $\left.\mathrm{eV}, 110{ }^{\circ} \mathrm{C}\right): 305(36)[\mathrm{M}]^{+}, 179(5), 166(100)$. HRMS $\left(\mathrm{C}_{13} \mathrm{H}_{18} \mathrm{~N}_{3} \mathrm{~F}_{3}\right)[\mathrm{M}]^{+}$: calc. 305.1346, found 305.1347. IR (ATR, ṽ): 3332, 3174, 2937, 2864, 1686, 1628, 1551, 1441, 1367, 1315, 1166, 917, 872, $824,722,671,578,520,471,443,417 \mathrm{~cm}^{-1}$.

\section{3-Isobutyl-5,6-dihydropyrazin-2(1H)-one (5de).}

According to general procedure $3,300 \mathrm{mg}$ of resin $3 \mathrm{~d}$ (batch $3 \mathrm{~d} .1,0.697 \mathrm{mmol} / \mathrm{g}$,<smiles>CC(C)CC1=NCCNC1=O</smiles>
$0.209 \mathrm{mmol})$ were converted with $41.0 \mathrm{mg}$ of ethane-1,2-diamine $(0.046 \mathrm{~mL}, 0.687$ mmol, 3.29 equiv.) in $4.0 \mathrm{~mL}$ of methanol to give $32.2 \mathrm{mg}(0.209 \mathrm{mmol})$ of the target substance in quantitative yield as a white solid. $\mathrm{R}_{f}$ (dichloromethane/ methanol, $10: 1)=0.32 .{ }^{1} \mathrm{H} \mathrm{NMR}\left(300 \mathrm{MHz}, \mathrm{CDCl}_{3}, \mathrm{ppm}\right), \delta=0.94(\mathrm{~d}, J=6.6 \mathrm{~Hz}, 6 \mathrm{H}), 2.01-2.13$ (m, $1 \mathrm{H}$ ), $2.47(\mathrm{~d}, J=7.2 \mathrm{~Hz}, 2 \mathrm{H}$ ), $3.42(\mathrm{td}, J=6.3 \mathrm{~Hz}, J=3.0 \mathrm{~Hz}, 2 \mathrm{H}$ ), $3.75(\mathrm{t}, J=6.2 \mathrm{~Hz}, 2 \mathrm{H}$ ), 7.07 (bs, $1 \mathrm{H}) .{ }^{13} \mathrm{C} \mathrm{NMR}\left(75 \mathrm{MHz}, \mathrm{CDCl}_{3}, \mathrm{ppm}\right), \delta=22.5,26.2,39.0,42.4,47.6,158.1,166.0 . \mathrm{El}(\mathrm{m} / \mathrm{z}, 70 \mathrm{eV}, 30$ $\left.{ }^{\circ} \mathrm{C}\right): 154(30)[\mathrm{M}]^{+}, 139$ (13), 125 (26), $112(100), 84$ (78). HRMS $\left(\mathrm{C}_{8} \mathrm{H}_{14} \mathrm{~N}_{2} \mathrm{O}\right)$ [M] $]^{+}$: calc. 154.1101, found 154.1099. IR (ATR, ṽ): 3189, 3070, 2950, 1684, 1628, 1465, 1416, 1362, 1335, 1283, 1165, 1101, $1072,1000,938,799,691,566,529,496,408 \mathrm{~cm}^{-1}$.

\section{3-(4-Hydroxyphenyl)-5,6-dihydropyrazin-2(1H)-one (5be).}

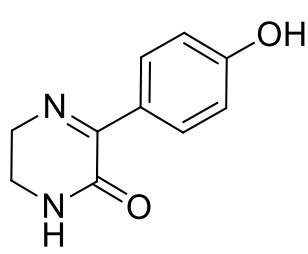

According to general procedure 3, $442 \mathrm{mg}$ of resin $3 \mathbf{b}$ (batch 3b.4, 0.696 $\mathrm{mmol} / \mathrm{g}, 0.308 \mathrm{mmol}$ ) were converted with $95.0 \mathrm{mg}$ of ethane-1,2-diamine ( $0.985 \mathrm{mmol}, 3.20$ equiv.) in $4.0 \mathrm{~mL}$ of methanol to give $50.5 \mathrm{mg}(0.266 \mathrm{mmol})$ of the target substance in $86 \%$ yield as a yellow solid. $R_{f}$ (dichloromethane/methanol, 10:1) $=0.32 .{ }^{1} \mathrm{H} \mathrm{NMR}(250 \mathrm{MHz}, \mathrm{MeOD}, \mathrm{ppm}), \delta=$ 3.39 - $3.47(\mathrm{~m}, 2 \mathrm{H}), 3.76-3.84(\mathrm{~m}, 2 \mathrm{H}), 6.74-6.84(\mathrm{~m}, 2 \mathrm{H}), 7.65-7.76(\mathrm{~m}, 2 \mathrm{H}) .{ }^{13} \mathrm{C} N M R(63 \mathrm{MHz}$, MeOD, ppm), $\delta=39.6,48.9,115.9,128.0,131.9,159.6,161.3,163.9 . \mathrm{FAB}(\mathrm{m} / \mathrm{z}): 190[\mathrm{M}]^{+}, 191$ $[\mathrm{M}+1]^{+}, 169,154,137,136$. HRMS $\left(\mathrm{C}_{10} \mathrm{H}_{10} \mathrm{~N}_{2} \mathrm{O}_{2}\right)\left[\mathrm{M}^{+}\right]$: calc. 191.0815, found 191.0817. IR (ATR, v): $3245,2933,2449,1655,1607,1509,1439,1339,1268,1235,1170,1103,1045,1004,838,728,710$, $639,568,533 \mathrm{~cm}^{-1}$.

\section{3-(4-Hydroxyphenyl)-6-methyl-5,6-dihydropyrazin-2(1H)-one and 3-(4-hydroxyphenyl)-5-methyl- 5,6-dihydropyrazin-2(1H)-one (5bf).}

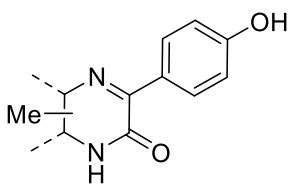

According to general procedure 3, $146 \mathrm{mg}$ of resin $3 \mathrm{~b}$ (batch 3b.1, 0.664 $\mathrm{mmol} / \mathrm{g}, 0.0969 \mathrm{mmol}$ ) were converted with $24.0 \mathrm{mg}$ of propane-1,2-diamine ( $0.325 \mathrm{mmol}, 3.37$ equiv.) in $4.0 \mathrm{~mL}$ of methanol to give $17.6 \mathrm{mg}(0.086 \mathrm{mmol})$ of the target substances as inseparable mixture of isomers in $89 \%$ yield as a yellow solid. $R_{f}$ (dichloromethane/methanol, 10:1) $=0.38$. Isomer 1:Isomer 2 (1:0.23, according to ${ }^{1} \mathrm{H}$-NMR integration). Isomer1: ${ }^{1} \mathrm{H}$ NMR (500 MHz, MeOD, ppm), $\delta=1.42(\mathrm{~d}, J=7.1 \mathrm{~Hz}, 3 \mathrm{H}$ ), 3.87 $3.96(\mathrm{~m}, 1 \mathrm{H}), 3.23(\mathrm{dd}, J=13.1 \mathrm{~Hz}, 9.4 \mathrm{~Hz}, 1 \mathrm{H}), 3.50(\mathrm{dd}, J=13.1,4.6 \mathrm{~Hz}, 1 \mathrm{H}), 6.83-6.86(\mathrm{~m}, 2.45 \mathrm{H})$, $7.73-7.76$ (m, $2.45 \mathrm{H}$ ). ${ }^{13} \mathrm{C} \mathrm{NMR} \mathrm{(100} \mathrm{MHz,} \mathrm{CDCl}_{3}$ and MeOD, ppm), $\delta=19.0,45.1,53.6,115.7,127.5$, 131.6, 159.2, 160.6, 162.2. Isomer 2: ${ }^{1} \mathrm{H}$ NMR (500 MHz, MeOD, ppm), $\delta=1.31$ (d, $J=6.6 \mathrm{~Hz}, 3 \mathrm{H}$ ), $3.43(\mathrm{dd}, J=16.1 \mathrm{~Hz}, J=5.4 \mathrm{~Hz}, 1 \mathrm{H}), 3.73-3.81(\mathrm{~m}, 1 \mathrm{H}), 3.99(\mathrm{dd}, J=16.1 \mathrm{~Hz}, J=4.2 \mathrm{~Hz}, 1 \mathrm{H}), 6.83-$ $6.86(\mathrm{~m}, 2 \mathrm{H}), 7.73-7.76(\mathrm{~m}, 2 \mathrm{H}) .{ }^{13} \mathrm{C} \mathrm{NMR}\left(100 \mathrm{MHz}, \mathrm{CDCl}_{3}\right.$ and $\left.\mathrm{MeOD}, \mathrm{ppm}\right), \delta=18.4,46.4,55.2$, 115.7, 127.4, 131.6, 159.4, 160.7, 163.2. El (m/z, $\left.70 \mathrm{eV}, 100^{\circ} \mathrm{C}\right): 204$ (86) [M] ${ }^{+}, 161$ (60), 147 (47), 133 
(58), 119 (100). HRMS $\left(\mathrm{C}_{11} \mathrm{H}_{12} \mathrm{~N}_{2} \mathrm{O}_{2}\right)$ [M]': calc. 204.0893, found 204.0895. IR (ATR, v): 3210, 2369, $1679,1600,1513,1446,1354,1310,1277,1182,1134,1084,1067,1021,838,800,724,621,596$, $547,501 \mathrm{~cm}^{-1}$.

\section{3-Phenyl-5,6-dihydropyrazin-2(1H)-one (5ce)}<smiles>O=C1NCCN=C1c1ccccc1</smiles>

According to general procedure 3, $1.00 \mathrm{~g}$ of resin $3 \mathrm{c}$ (batch 3c.2, $1.00 \mathrm{~g}, 0.784 \mathrm{~mol} / \mathrm{g}$ ) were converted with ethane-1,2-diamine (147 mg, $2.45 \mathrm{mmol}, 3.00$ equiv.) in 7.00 $\mathrm{mL}$ of methanol. The suspension was filtered through a syringe phase separator, the resin washed with methanol and the solvent of the filtrate removed under reduced pressure. The crude product was purified by flash chromatography (30:1, dichloromethane:methanol) to yield an off white solid in quant. yield. $R_{f}$ (dichloromethane/methanol, 50:1) $=0.19 .{ }^{1} \mathrm{H}$ NMR $\left(500 \mathrm{MHz}, \mathrm{CDCl}_{3}, \mathrm{ppm}\right) \delta=7.89(\mathrm{~d}, J=6.7 \mathrm{~Hz}, 2$ H), $7.47-7.37(\mathrm{~m}, 3 \mathrm{H}), 3.95(\mathrm{t}, J=6.3 \mathrm{~Hz}, 2 \mathrm{H}), 3.50(\mathrm{td}, J=6.3, J=3.5 \mathrm{~Hz}, 2 \mathrm{H}) .{ }^{13} \mathrm{C} \mathrm{NMR}(126 \mathrm{MHz}$, $\left.\mathrm{CDCl}_{3}, \mathrm{ppm}\right) \delta=162.4,157.8,135.3,130.5,128.9,128.2,48.4,39.2$. IR (ATR): $\tilde{\mathrm{v}}=3178,3069,2969$, 2870, 1677, 1606, 1491, 1441, 1407, 1344, 1256, 1220, 1153, 1110, 1080, 1000, 976, 935, 871, 806, $772,732,714,698,577,531,472 \mathrm{~cm}^{-1} . \mathrm{El}\left(\mathrm{m} / \mathrm{z}, 70 \mathrm{eV}, 60^{\circ} \mathrm{C}\right): 174$ [M]+ (18), 137 (10), 125 (100), 117 (24). - HRMS $\left(\mathrm{C}_{10} \mathrm{H}_{10} \mathrm{ON}_{2}\right)$ : calc.: 174.0788; found: 174.0789 .

\section{5-Methyl-3-phenyl-5,6-dihydropyrazin-2(1H)-one and 5-methyl-3-phenyl-5,6-dihydropyrazin-2(1H)- one (5cf)}<smiles>CC1N=C(c2ccccc2)C(=O)NC1C</smiles>

According to general procedure 3, $500 \mathrm{mg}$ of resin 3c (batch 3c.2, $0.784 \mathrm{~mol} / \mathrm{g}$ ) were converted with propane-1,2-diamine $(85.0 \mathrm{mg}, 1.18 \mathrm{mmol}, 3.00$ equiv.) in $5.00 \mathrm{~mL}$ of methanol. The suspension was filtered through a syringe phase separator, the resin washed with methanol and the solvent of the filtrate removed under reduced pressure. The crude product was purified by flash chromatography (50:1, DCM:MeOH) to yield $60.6 \mathrm{mg}$ of a white solid in $84 \%$ yield as an iseparable mixture of isomers. $R_{f}$ (dichloromethane/methanol, 50:1) $=0.20 .{ }^{1} \mathrm{H}$ NMR (500 $\mathrm{MHz}$, Chloroform-d, ppm) $\delta=7.89(\mathrm{~d}, J=6.6 \mathrm{~Hz}, 2 \mathrm{H}), 7.49-7.35(\mathrm{~m}, 3 \mathrm{H}), 7.03(\mathrm{~s}, 1 \mathrm{H}), 6.73(\mathrm{~s}, 0.7$ H), $4.19-4.05(m, 0.2 H), 4.02-3.92(m, 0.8 H), 3.87-3.72(m, 0.2 H), 3.55-3.46(m, 1 H), 3.31-$ $3.22(\mathrm{~m}, 0.8 \mathrm{H}), 1.43(\mathrm{~d}, J=6.9 \mathrm{~Hz}, 2.3 \mathrm{H}), 1.30(\mathrm{~d}, J=6.5 \mathrm{~Hz}, 0.7 \mathrm{H}) .{ }^{13} \mathrm{C} \mathrm{NMR}\left(126 \mathrm{MHz}, \mathrm{CDCl}_{3}, \mathrm{ppm}\right)$ $\delta=161.8,160.7,157.8,157.8,135.2,135.0,130.4,130.3,128.8,128.8,128.0,55.1,53.1,54.7,44.8$, 19.9, 18.5. IR (ATR): $\tilde{v}=3199,2966,1668,1592,1571,1473,1443,1372,1357,1314,1241,1179$, 1105, 1069, 999, 936, 806, 736, 693, 620, 578, 542, 524, 492, 447, $403 \mathrm{~cm}^{-1}$. El (m/z, $\left.70 \mathrm{eV}, 60{ }^{\circ} \mathrm{C}\right)$ : $189[\mathrm{M}+\mathrm{H}]^{+}(11), 188[\mathrm{M}]^{+}(84), 145(54), 131(46), 130(16), 117(45), 104(100), 103(25), 77$ (15), 69 (16). HRMS $\left(\mathrm{C}_{11} \mathrm{H}_{12} \mathrm{ON}_{2}\right)$ : calc.: 188.0944; found: 188.0943 . EA calc.: C: $70.19, \mathrm{H}: 6.43, \mathrm{~N}: 14.88$; found: C: $69.83, \mathrm{H}: 6.41, \mathrm{~N}: 14.61$.

\section{3-Methyl-2H-benzo[b][1,4]oxazin-2-one (7aa)}<smiles>Cc1nc2ccccc2oc1=O</smiles>

According to general procedure 3, $300 \mathrm{mg}$ of resin 3a (batch 3a.1, $0.745 \mathrm{mmol} / \mathrm{g}$, $0.224 \mathrm{mmol})$ were converted with $81.0 \mathrm{mg}$ of 2 -aminophenol $(0.740 \mathrm{mmol}$, 3.30 equiv.) in $4.0 \mathrm{~mL}$ of methanol to give $12.4 \mathrm{mg}(0.077 \mathrm{mmol})$ of the target substance in $34 \%$ yield as a light yellow solid. $R_{f}$ (cyclohexane/ethyl acetate, $\left.10: 1\right)=0.33 .{ }^{1} \mathrm{H}$ NMR (300 MHz, CDCl 3 ppm), $\delta=2.56(\mathrm{~s}, 3 \mathrm{H}), 7.26(\mathrm{dd}, J=8.2 \mathrm{~Hz}, J=1.2 \mathrm{~Hz}, 1 \mathrm{H}), 7.30-7.37(\mathrm{~m}, 1 \mathrm{H}), 7.41$ - $7.50(\mathrm{~m}, 1 \mathrm{H}), 7.69$ (dd, $J=7.9 \mathrm{~Hz}, J=1.8 \mathrm{~Hz}, 1 \mathrm{H}) .{ }^{13} \mathrm{C} \mathrm{NMR}\left(63 \mathrm{MHz}, \mathrm{CDCl}_{3}, \mathrm{ppm}\right), \delta=21.3,116.3$, 125.4, 128.6, 130.5, 131.1, 146.5, 153.2, 155.1. El (m/z, $\left.70 \mathrm{eV}, 50{ }^{\circ} \mathrm{C}\right): 161$ (58) [M] ${ }^{+}, 133$ (100), 105 (13), 104 (10), 78 (11), 64 (18), 63 (24). HRMS $\left(\mathrm{C}_{9} \mathrm{H}_{14} \mathrm{~N}_{2} \mathrm{O}\right)$ [M] $]^{+}$: calc. 161.0471 found 161.0469. IR (ATR, v): 2923, 1723, 1612, 1573, 1477, 1454, 1370, 1282, 1219, 1147, 1118, 1091, 1004, 956, 916, $849,779,754,725,595,563,466,397 \mathrm{~cm}^{-1}$. EA $\left(\mathrm{C}_{9} \mathrm{H}_{7} \mathrm{NO}_{2}\right)$ : calc. C $67.07, \mathrm{H} 4.38, \mathrm{~N} 8.69$; found. C 67.07, H 4.46, N 8.59. 


\section{6-(tert-Butyl)-3-methyl-2H-benzo[b][1,4]oxazin-2-one (7ab)}<smiles>Cc1nc2cc(C(C)(C)C)ccc2oc1=O</smiles>

According to general procedure $3,300 \mathrm{mg}$ of resin 3a (batch 3a.1, 0.745 $\mathrm{mmol} / \mathrm{g}, 0.224 \mathrm{mmol}$ ) were converted with $122 \mathrm{mg}$ of 2-amino-4tertbutylphenol ( $0.740 \mathrm{mmol}, 3.30$ equiv.) in $4.0 \mathrm{~mL}$ of methanol to give 25.8 $\mathrm{mg}(0.119 \mathrm{mmol})$ of the target substance in $53 \%$ yield as a light brown solid. $\mathrm{R}_{f}$ (cyclohexane/ethyl acetate, 4:1) $=0.67 .{ }^{1} \mathrm{H} \mathrm{NMR}\left(500 \mathrm{MHz}, \mathrm{CDCl}_{3}, \mathrm{ppm}\right), \delta=1.36(\mathrm{~s}, 9 \mathrm{H}), 2.57(\mathrm{~s}, 3$ H), $7.21(\mathrm{~d}, J=8.8 \mathrm{~Hz}, 1 \mathrm{H}), 7.50(\mathrm{dd}, J=8.6 \mathrm{~Hz}, J=2.4 \mathrm{~Hz}, 1 \mathrm{H}), 7.71(\mathrm{~d}, J=2.2 \mathrm{~Hz}, 1 \mathrm{H}) .{ }^{13} \mathrm{C} \mathrm{NMR}(125$ $\left.\mathrm{MHz}, \mathrm{CDCl}_{3}, \mathrm{ppm}\right), \delta=21.3,31.3,34.6,115.7,125.1,128.0,130.6,144.3,148.9,153.5,154.8 \mathrm{.} \mathrm{El}(\mathrm{m} / \mathrm{z}$, $\left.70 \mathrm{eV}, 40^{\circ} \mathrm{C}\right): 217(100)[\mathrm{M}]^{+}, 202(14), 174(100), 146(7), 133$ (8). HRMS $\left(\mathrm{C}_{13} \mathrm{H}_{15} \mathrm{NO}_{2}\right)[\mathrm{M}]^{+}$: calc. 217.1097, found 217.1099. IR (ATR, ̃): 2960, 1735, 1614, 1568, 1490, 1416, 1367, 1363, 1260, 1230, $1141,1102,1091,1013,936,890,840,764,642,574,556,495,457,390 \mathrm{~cm}^{-1}$. EA $\left(\mathrm{C}_{13} \mathrm{H}_{15} \mathrm{NO}_{2}\right)$ : calc. C 71.87, H 6.96, N 6.45; found. C 71.15, H 6.87, N 6.41.

\section{3-(4-Hydroxyphenyl)-2H-benzo[b][1,4]oxazin-2-one (7ba)}

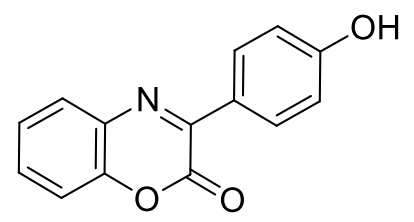

According to general procedure $3,300 \mathrm{mg}$ of resin $\mathbf{3 b}$ (batch $3 \mathrm{~b} .4,0.696$ $\mathrm{mmol} / \mathrm{g}, 0.209 \mathrm{mmol}$ ) were converted with $73.0 \mathrm{mg}$ of 2-aminophenol ( $0.669 \mathrm{mmol}, 3.26$ equiv.) in $4.0 \mathrm{~mL}$ of methanol to give $31.0 \mathrm{mg}(0.130$ $\mathrm{mmol}$ ) of the target substance in $62 \%$ yield as a deep yellow solid. $\mathrm{R}_{f}$ (cyclohexane/ethyl acetate, 2:1) $=0.47 .{ }^{1} \mathrm{H} \mathrm{NMR}(300 \mathrm{MHz}, \mathrm{MeOD}, \mathrm{ppm})$, $\delta=6.83-6.90(\mathrm{~m}, 2 \mathrm{H}), 7.28-7.42(\mathrm{~m}, 2 \mathrm{H}), 7.46-7.55(\mathrm{~m}, 1 \mathrm{H}), 7.77(\mathrm{dd}, J=7.9 \mathrm{~Hz}, J=1.5 \mathrm{~Hz}, 1 \mathrm{H})$, 8.22 - 8.30 (m, $2 \mathrm{H}$ ). ${ }^{13} \mathrm{C}$ NMR (63 MHz, MeOD, ppm), $\delta=116.2,117.1,126.6,127.3,129.9,131.5$, 132.8, 133.2, 147.9, 151.8, 154.1, 162.2. El (m/z, $\left.70 \mathrm{eV}, 110{ }^{\circ} \mathrm{C}\right): 239$ (63) [M] ${ }^{+}, 211$ (100), 149 (60), 133 (43). HRMS $\left(\mathrm{C}_{14} \mathrm{H}_{9} \mathrm{NO}_{3}\right)[\mathrm{M}]^{+}$: calc. 239.0582, found 239.0583. IR (ATR, ṽ): 3373, 3293, 2922, 1733, $1603,1582,1510,1470,1444,1302,1246,1156,1024,979,918,838,744,670,568,532,455 \mathrm{~cm}^{-1}$.

\section{6-(tert-Butyl)-3-(4-hydroxyphenyl)-2H-benzo[b][1,4]oxazin-2-one (7bb)}<smiles>CC(C)(C)c1ccc2oc(=O)c(-c3ccc(O)cc3)nc2c1</smiles>

According to general procedure $3,300 \mathrm{mg}$ of resin $3 \mathbf{b}$ (batch $3 \mathrm{~b} .2$, $0.7054 \mathrm{mmol} / \mathrm{g}, 0.212 \mathrm{mmol}$ ) were converted with $111 \mathrm{mg}$ of 2amino-4-(tert-butyl)phenol ( $0.669 \mathrm{mmol}, 3.16$ equiv.) in $4.0 \mathrm{~mL}$ of methanol to give $53.3 \mathrm{mg}(0.180 \mathrm{mmol}, 295.3 \mathrm{~g} / \mathrm{mol})$ of the target substance in $85 \%$ yield as a brown solid. $\mathrm{R}_{f}$ (cyclohexane/ethyl acetate, 2:1) = 0.54. ${ }^{1} \mathrm{H} \mathrm{NMR}(300 \mathrm{MHz}, \mathrm{MeOD}, \mathrm{ppm}), \delta=\mathrm{ppm} 1.42(\mathrm{~s}, 9 \mathrm{H}), 6.95-7.01(\mathrm{~m}, 1 \mathrm{H}), 7.30$ (d, $J=1.5 \mathrm{~Hz}, 2 \mathrm{H}$ ), $7.56(\mathrm{dd}, J=8.6 \mathrm{~Hz}, J=2.4 \mathrm{~Hz}, 1 \mathrm{H}), 7.85(\mathrm{~d}, J=2.3 \mathrm{~Hz}, 1 \mathrm{H}), 8.33-8.39(\mathrm{~m}, 2 \mathrm{H})$. ${ }^{13} \mathrm{C} \mathrm{NMR}(63 \mathrm{MHz}, \mathrm{MeOD}, \mathrm{ppm}), \delta=31.8,35.3,115.9,116.1,126.1,126.6,128.8,132.0,132.2,144.8$, 149.8, 150.8, 154.0, 161.3. El (m/z, $\left.70 \mathrm{eV}, 130{ }^{\circ} \mathrm{C}\right): 295$ (41) [M] ${ }^{+}, 267$ (18), 252 (100). HRMS $\left(\mathrm{C}_{18} \mathrm{H}_{17} \mathrm{NO}_{3}\right)[\mathrm{M}]^{+}$: calc. 295.1208, found 295.1211. IR (ATR, ṽ): 2953, 1732, 1602, 1528, 1508, 1489, $1459,1419,1366,1311,1255,1219,1165,1123,1110,1064,1030,969,882,853,828,809,782$, $759,694,664,640,628,612,536,469,452 \mathrm{~cm}^{-1}$.

\section{6-Chloro-3-(4-hydroxyphenyl)-2H-benzo[b][1,4]oxazin-2-one (7bc)}<smiles>O=c1oc2ccc(Cl)cc2nc1-c1ccc(O)cc1</smiles>

According to general procedure $3,300 \mathrm{mg}$ of resin $\mathbf{3 b}$ (batch $3 \mathrm{~b} .4$, $0.696 \mathrm{mmol} / \mathrm{g}, 0.209 \mathrm{mmol}$ ) were converted with $96.0 \mathrm{mg}$ of 2 amino-4-chlorophenol $(0.669 \mathrm{mmol}, 3.20$ equiv.) in $4.0 \mathrm{~mL}$ of methanol to give $36.2 \mathrm{mg}(0.132 \mathrm{mmol})$ of the target substance in $63 \%$ yield as an orange solid. $R_{f}$ (cyclohexane/ethyl acetate, $2: 1$ ) $=$ 0.55. ${ }^{1} \mathrm{H}$ NMR (250 MHz, MeOD, ppm), $\delta=6.82-6.90(\mathrm{~m}, 2 \mathrm{H}), 7.27(\mathrm{~d}, J=8.9 \mathrm{~Hz}, 1 \mathrm{H}), 7.44(\mathrm{dd}, J=$ 
$8.9 \mathrm{~Hz}, J=2.4 \mathrm{~Hz}, 1 \mathrm{H}), 7.74(\mathrm{~d}, J=2.4 \mathrm{~Hz}, 1 \mathrm{H}), 8.22-8.30(\mathrm{~m}, 2 \mathrm{H}) .{ }^{13} \mathrm{C}$ NMR $(63 \mathrm{MHz}, \mathrm{MeOD}, \mathrm{ppm}), \delta$ $=116.2,118.2,126.5,129.0,130.9,131.3,132.8,133.5,146.1,152.3,153.3,162.3 . \mathrm{El}(\mathrm{m} / \mathrm{z}, 70 \mathrm{eV}$, $\left.100{ }^{\circ} \mathrm{C}\right): 273 / 275(13 / 4)[\mathrm{M}]^{+}, 245 / 247(42 / 12), 214(4), 155$ (24), $143(24) . \mathrm{HRMS}\left(\mathrm{C}_{14} \mathrm{H}_{8} \mathrm{NClO}_{3}\right)[\mathrm{M}]^{+}:$ calc. 273.0193 , found 273.0190. IR (ATR, ṽ): 3341, 3085, 2489, 1710, 1598, 1581, 1508, 1464, 1436, $1299,1274,1239,1217,1174,1138,1069,978,929,873,839,825,665,634,586,543,527,453 \mathrm{~cm}^{-1}$.

\section{3-(4-Hydroxyphenyl)-6-phenyl-2H-benzo[b][1,4]oxazin-2-one (7bd)}<smiles>O=c1oc2ccc(-c3ccccc3)cc2nc1-c1ccc(O)cc1</smiles>

According to general procedure 3, $266 \mathrm{mg}$ of resin $\mathbf{3 b}$ (batch $3 \mathrm{~b} .2,0.705 \mathrm{mmol} / \mathrm{g}, 0.188 \mathrm{mmol}$ ) were converted with $114 \mathrm{mg}$ of 3-amino-biphenylol ( $0.616 \mathrm{mmol}, 3.28$ equiv.) in $4.0 \mathrm{~mL}$ of methanol to give $29.5 \mathrm{mg}(0.094 \mathrm{mmol})$ of the target substance in $50 \%$ yield as a brown solid. $\mathrm{R}_{f}$ (cyclohexane/ethyl acetate, $2: 1$ ) $=0.50 .{ }^{1} \mathrm{H}$ NMR $(250 \mathrm{MHz}, \mathrm{MeOD}, \mathrm{ppm}), \delta=6.84-6.94(\mathrm{~m}, 2 \mathrm{H}), 7.30-7.48(\mathrm{~m}, 4 \mathrm{H}), 7.56-7.69(\mathrm{~m}, 3$ H), $7.97\left(\mathrm{~d}, J=2.4 \mathrm{~Hz}, 1 \mathrm{H}\right.$ ), $8.22-8.29(\mathrm{~m}, 2 \mathrm{H}) .{ }^{13} \mathrm{C} \mathrm{NMR}(63 \mathrm{MHz}, \mathrm{MeOD}, \mathrm{ppm}), \delta=115.6,116.5$, 125.9, 127.2, 127.3, 128.0, 129.2, 129.3, 131.8, 132.1, 139.2, 139.6, 145.7, 150.7, 153.0, 160.8. El (m/z, $\left.70 \mathrm{eV}, 130^{\circ} \mathrm{C}\right): 315$ (8) [M] ${ }^{+}, 287$ (24), 139 (12), 123 (11), 105 (19). HRMS ( $\left.\mathrm{C}_{20} \mathrm{H}_{13} \mathrm{NO}_{3}\right)$ [M+]: calc. 315.0895, found 315.0898. IR (ATR, ṽ): 3409, 2953, 2919, 2850, 1721, 1602, 1579, 1510, 1489, 1461, $1365,1341,1279,1209,1176,1145,1111,1088,985,944,885,839,814,719,665,634,558,528$, $491,473,456,432,409 \mathrm{~cm}^{-1}$.

\section{3-(4-Hydroxyphenyl)-7-methyl-2H-benzo[b][1,4]oxazin-2-one (7be)}<smiles>Cc1ccc2nc(-c3ccc(O)cc3)c(=O)oc2c1</smiles>

According to general procedure $3,300 \mathrm{mg}$ of resin $\mathbf{3 b}$ (batch $3 \mathrm{~b} .2$, $0.705 \mathrm{mmol} / \mathrm{g}, 0.212 \mathrm{mmol}$ ) were converted with $82.0 \mathrm{mg}$ of 2 -amino5-methylphenol ( $0.669 \mathrm{mmol}, 3.16$ equiv.) in $4.0 \mathrm{~mL}$ of methanol to give $36.6 \mathrm{mg}(0.145 \mathrm{mmol})$ of the target substance in $68 \%$ yield as a deep yellow solid. $\mathrm{R}_{f}$ (cyclohexane/ethyl acetate, 2:1) $=0.48$. ${ }^{1} \mathrm{H}$ NMR (500 MHz, CDCl 3 and MeOD, ppm), $\delta=2.26(\mathrm{~s}, 3 \mathrm{H}), 6.69-6.71(\mathrm{~m}, 2 \mathrm{H}), 6.90(\mathrm{~s}, 1 \mathrm{H}), 6.98(\mathrm{~d}, J=8.2$ $\mathrm{Hz}, 1 \mathrm{H}), 7.45(\mathrm{~d}, J=8.2 \mathrm{~Hz}, 1 \mathrm{H}), 8.01-8.04(\mathrm{~m}, 2 \mathrm{H}) .{ }^{13} \mathrm{C} \mathrm{NMR}\left(125 \mathrm{MHz}, \mathrm{CDCl}_{3}\right.$ and MeOD, ppm), $\delta=$ 21.0, 114.8, 115.7, 125.4, 126.3, 128.0, 129.4, 130.9, 141.4, 145.7, 148.7, 152.8, 159.9. El (m/z, $70 \mathrm{eV}$, $\left.130{ }^{\circ} \mathrm{C}\right): 253(66)[\mathrm{M}]^{+}, 225(100), 196(11)$. $\mathrm{HRMS}\left(\mathrm{C}_{15} \mathrm{H}_{11} \mathrm{NO}_{3}\right)[\mathrm{M}+\mathrm{H}]$ : calc. 253.0733, found 253.0731. IR (ATR, v): 3245, 1731, 1708, 1691, 1601, 1582, 1505, 1443, 1370, 1314, 1291, 1233, 1175, 1136, $1113,1015,977,949,861,842,809,720,653,603,588,558,530,498,469,452,414 \mathrm{~cm}^{-1}$.

\section{6-(tert-Butyl)-3-phenyl-2H-benzo[b][1,4]oxazin-2-one (7cb)}<smiles>CC(C)(C)c1ccc2oc(=O)c(-c3ccccc3)nc2c1</smiles>

According to general procedure 3, $300 \mathrm{mg}$ of resin 3c (batch 3c.1, 0.708 $\mathrm{mmol} / \mathrm{g}, 0.212 \mathrm{mmol}$ ) were converted with $111 \mathrm{mg}$ of 2-amino-4tertbutylphenol $(0.669 \mathrm{mmol}, 3.13$ equiv. $)$ in $4.0 \mathrm{~mL}$ of methanol to give $15.1 \mathrm{mg}(0.054 \mathrm{mmol})$ of the target substance in $26 \%$ yield as a deep brown solid. $\mathrm{R}_{f}$ (cyclohexane/ethyl acetate, $\left.2: 1\right)=0.77 .{ }^{1} \mathrm{H} \mathrm{NMR} \mathrm{(400} \mathrm{MHz,} \mathrm{CDCl}_{3}, \mathrm{ppm}$ ), $\delta=1.40$ (s, 9 H), $7.26-7.31(\mathrm{~m}, 1 \mathrm{H}), 7.50-7.60(\mathrm{~m}, 4 \mathrm{H}), 7.87$ (br. s., $1 \mathrm{H}), 8.31-8.36(\mathrm{~m}, 2 \mathrm{H}) .{ }^{13} \mathrm{C} \mathrm{NMR}(100 \mathrm{MHz}$, $\left.\mathrm{CDCl}_{3}, \mathrm{ppm}\right), \delta=31.3,34.7,115.5,126.0,128.4,128.7,129.3,131.3,134.3,144.2,149.0,150.7$, 152.6. El (m/z, $\left.70 \mathrm{eV}, 80^{\circ} \mathrm{C}\right): 279$ (25) [M] ${ }^{+}, 251$ (21), 236 (100), 208 (7). HRMS $\left(\mathrm{C}_{18} \mathrm{H}_{17} \mathrm{NO}_{2}\right)\left[\mathrm{M}^{+}\right]$: calc. 279.1254, found 279.1253. IR (ATR, ṽ): 2954, 1738, 1592, 1488, 1445, 1364, 1300, 1228, 1129, 1090, $1000,972,890,814,800,753,732,684,659,641,581,520,488,414 \mathrm{~cm}^{-1}$. 


\section{3-Isobutyl-2H-benzo[b][1,4]oxazin-2-one (7da)}<smiles>CC(C)Cc1nc2ccccc2oc1=O</smiles>

According to general procedure $3,300 \mathrm{mg}$ of resin $3 \mathrm{~d}$ (batch $3 \mathrm{~d} .3,0.797$ $\mathrm{mmol} / \mathrm{g}, 0.239 \mathrm{mmol}$ ) were converted with $78.0 \mathrm{mg}$ of 2-amino-phenol $(0.716$ mmol, 3.00 equiv.) in $4.0 \mathrm{~mL}$ of methanol to give $18.0 \mathrm{mg}(0.089 \mathrm{mmol})$ of the target substance in $37 \%$ yield as a brown solid. $\mathrm{R}_{f}$ (cyclohexane/ethyl acetate, 10:1) $=0.36 .{ }^{1} \mathrm{H}$ NMR $\left(500 \mathrm{MHz}\right.$, methanol-d $\left.\mathrm{d}_{4}, \mathrm{ppm}\right), \delta=1.01(\mathrm{~d}, J=6.7 \mathrm{~Hz}, 6 \mathrm{H}), 2.23-2.33(\mathrm{~m}, 1 \mathrm{H})$, $2.67-2.73(\mathrm{~m}, 2 \mathrm{H}), 7.30(\mathrm{dt}, J=8.2 \mathrm{~Hz}, J=1.6 \mathrm{~Hz}, 1 \mathrm{H}), 7.35-7.40(\mathrm{~m}, 1 \mathrm{H}), 7.48-7.53(\mathrm{~m}, 1 \mathrm{H}), 7.69$ - $7.73(\mathrm{~m}, 1 \mathrm{H}) .{ }^{13} \mathrm{C}$ NMR $\left(125 \mathrm{MHz}\right.$, methanol-d $\left.\mathrm{d}_{4}, \mathrm{ppm}\right), \delta=23.1(2 \mathrm{C}), 27.7,43.9,117.5,126.6,129.8$, 131.8, 132.6, 148.0, 154.6, 159.2. GCMS (m/z): 205 (11) [M] ${ }^{+}, 190$ (6), 163 (32), 135 (100). Raman (र̃): $3068,1748,1595,1538,1484,1449,1333,1257,1159,1131,1035,1003,977,463 \mathrm{~cm}^{-1}$.

\section{6-(tert-Butyl)-3-isobutyl-2H-benzo[b][1,4]oxazin-2-one (7db)}

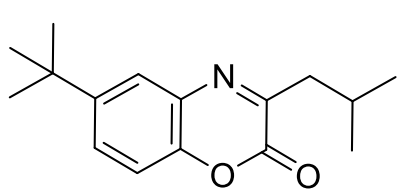

According to general procedure $3,300 \mathrm{mg}$ of resin $3 \mathrm{~d}$ (batch $3 \mathrm{~d} .2,0.789$ $\mathrm{mmol} / \mathrm{g}, 0.237 \mathrm{mmol}$ ) were converted with $111 \mathrm{mg}$ of 2-amino-4tertbutylphenol $(0.669 \mathrm{mmol}, 2.82$ equiv.) in $4.0 \mathrm{~mL}$ of methanol to give $23.2 \mathrm{mg}(0.089 \mathrm{mmol})$ of the target substance in $38 \%$ yield as a yellow solid. $\mathrm{R}_{f}$ (cyclohexane/ethyl acetate, 2:1) $\left.=0.84 .{ }^{1} \mathrm{H} \mathrm{NMR} \mathrm{(400} \mathrm{MHz,} \mathrm{CDCl}_{3}, \mathrm{ppm}\right), \delta=1.05-1.10$ (m, 6 H), $1.42(\mathrm{~d}, J=6.6 \mathrm{~Hz}, 9 \mathrm{H}), 2.25(\mathrm{~m}, 1 \mathrm{H}), 2.80-2.85(\mathrm{~m}, 2 \mathrm{H}), 7.22-7.35(\mathrm{~m}, 1 \mathrm{H}), 7.52-7.61(\mathrm{~m}, 1$ H), $7.79(\mathrm{~d}, J=4.0 \mathrm{~Hz}, 1 \mathrm{H}) .{ }^{13} \mathrm{C} \mathrm{NMR}\left(100 \mathrm{MHz} \mathrm{CDCl}_{3}, \mathrm{ppm}\right), \delta=22.6,26.8,31.3,34.6,42.9,115.7$, 125.4, 128.0, 130.7, 144.2, 148.8, 153.4, 157.4. El (m/z, $\left.70 \mathrm{eV}, 60^{\circ} \mathrm{C}\right): 259$ (25) [M] ${ }^{+}, 232$ (3), 216 (100), 189 (9), 173 (4). HRMS $\left(\mathrm{C}_{16} \mathrm{H}_{21} \mathrm{NO}_{2}\right)$ [M] $]^{+}$: calc. 259.1567, found 259.1566. IR (ATR, v): 2959, 2915, 2848, 1737, 1613, 1492, 1464, 1365, 1238, 1139, 1062, 1022, 944, 891, 823, 719, 642, 598, $564,452 \mathrm{~cm}^{-1}$.

\section{2,2,2-Trifluoro-N-(3-(2-oxo-2H-benzo[b][1,4]oxazin-3-yl)propyl)acetamide (7ea)}<smiles>O=C(NCCCc1nc2ccccc2oc1=O)C(F)(F)F</smiles>

According to general procedure $3,300 \mathrm{mg}$ of resin $3 \mathrm{e}$ (batch $3 \mathrm{e} .2$, $0.726 \mathrm{mmol} / \mathrm{g}, 0.218 \mathrm{mmol}$ ) were converted with $70.0 \mathrm{mg}$ of $2-$ aminophenol ( $0.640 \mathrm{mmol}, 2.94$ equiv.) in $4.0 \mathrm{~mL}$ of methanol to give $30.5 \mathrm{mg}(0.102 \mathrm{mmol})$ of the target substance in $47 \%$ yield as a white solid. $\mathrm{R}_{f}$ (cyclohexane/ethyl acetate, $2: 1$ ) $=0.39 .{ }^{1} \mathrm{H} \mathrm{NMR}\left(500 \mathrm{MHz}, \mathrm{CDCl}_{3}, \mathrm{ppm}\right.$ ), $\delta=2.12-2.19$ $(\mathrm{m}, 2 \mathrm{H}), 3.03(\mathrm{t}, J=6.9 \mathrm{~Hz}, 2 \mathrm{H}), 3.53(\mathrm{q}, J=6.5 \mathrm{~Hz}, 2 \mathrm{H}), 7.02$ (bs, $1 \mathrm{H}), 7.32$ (dd, $J=8.2 \mathrm{~Hz}, J=0.9 \mathrm{~Hz}$, $1 \mathrm{H}), 7.40$ (td, $J=7.7 \mathrm{~Hz}, J=1.3 \mathrm{~Hz}, 1 \mathrm{H}), 7.50-7.54(\mathrm{~m}, 1 \mathrm{H}), 7.74(\mathrm{dd}, J=7.9 \mathrm{~Hz}, J=1.6 \mathrm{~Hz}, 1 \mathrm{H}) .{ }^{13} \mathrm{C}$ $\operatorname{NMR}\left(125 \mathrm{MHz}, \mathrm{CDCl}_{3}, \mathrm{ppm}\right), \delta=24.6,31.1,39.6,115.9$ (q, $\left.J=288 \mathrm{~Hz}\right), 116.5,125.7,128.7,130.8$, 131.1, 146.4, 153.1, 156.6, 157.3 (q, $J=37.2 \mathrm{~Hz}) .{ }^{19} \mathrm{~F} \mathrm{NMR}\left(376 \mathrm{MHz}, \mathrm{CDCl}_{3}, \mathrm{ppm}\right), \delta=-75.8 . \mathrm{El}(\mathrm{m} / \mathrm{z}$, $\left.70 \mathrm{eV}, 80^{\circ} \mathrm{C}\right): 300$ (38) [M] ${ }^{+}, 187$ (51), 174 (48), 161 (22), 146 (100). HRMS $\left(\mathrm{C}_{13} \mathrm{H}_{11} \mathrm{~N}_{2} \mathrm{~F}_{2} \mathrm{O}_{3}\right.$ ) [M]: calc. 300.0716, found 300.0717. IR (ATR, ṽ): 3300, 2922, 1737, 1697, 1610, 1562, 1462, 1280, 1157, 1094, $1043,1010,918,898,756,707,626,561,522,463,404 \mathrm{~cm}^{-1}$.

\section{$\mathrm{N}$-(3-(6-(tert-butyl)-2-oxo-2H-benzo[b][1,4]oxazin-3-yl)propyl)-2,2,2-trifluoroacetamide (7eb).}<smiles>CC(C)(C)c1ccc2oc(=O)c(CCCNC(=O)C(F)(F)F)nc2c1</smiles>

According to general procedure $3,463 \mathrm{mg}$ of resin $3 \mathrm{e}$ (batch $3 e .1,0.709 \mathrm{mmol} / \mathrm{g}, 0.328 \mathrm{mmol})$ were converted with $163 \mathrm{mg}$ of 2-amino-4-tertbutylphenol $(0.988 \mathrm{mmol}$, 3.01 equiv.) in $4.0 \mathrm{~mL}$ of methanol to give $75.9 \mathrm{mg}$ $(0.213 \mathrm{mmol})$ of the target substance in $65 \%$ yield as a light yellow solid. $\mathrm{R}_{f}$ (cyclohexane/ethyl acetate, $2: 1)=0.61 .{ }^{1} \mathrm{H} \mathrm{NMR}\left(400 \mathrm{MHz}, \mathrm{CDCl}_{3}, \mathrm{ppm}\right), \delta=1.35-1.42(\mathrm{~m}, 9 \mathrm{H}), 2.16(\mathrm{t}, J=6.7 \mathrm{~Hz}, 2 \mathrm{H})$, $3.03(\mathrm{t}, J=6.8 \mathrm{~Hz}, 2 \mathrm{H}), 3.50-3.55(\mathrm{~m}, 2 \mathrm{H}), 7.22-7.29(\mathrm{~m}, 2 \mathrm{H}), 7.56(\mathrm{dd}, J=8.8 \mathrm{~Hz}, J=2.3 \mathrm{~Hz}, 1 \mathrm{H})$, $7.73(\mathrm{~d}, J=2.3 \mathrm{~Hz}, 1 \mathrm{H}) .{ }^{13} \mathrm{C} \mathrm{NMR}\left(100 \mathrm{MHz}, \mathrm{CDCl}_{3}, \mathrm{ppm}\right), \delta=24.6,31.2,31.3,34.7,39.7,115.9,115.9$ 
(q, $J=288 \mathrm{~Hz}$ ), 125.2, 128.6, 130.2, 144.1, 149.3, 153.4, 156.3, 157.3 (q, $J=38 \mathrm{~Hz}) .{ }^{19} \mathrm{~F} \mathrm{NMR} \mathrm{(376}$ $\left.\mathrm{MHz}, \mathrm{CDCl}_{3}, \mathrm{ppm}\right), \delta=-75.73 . \mathrm{El}\left(\mathrm{m} / \mathrm{z}, 70 \mathrm{eV}, 80^{\circ} \mathrm{C}\right): 356$ (94) [M] ${ }^{+}, 313$ (16), $246(32), 243$ (48), 230 (47), 217 (12), 202 (100). HRMS $\left(\mathrm{C}_{17} \mathrm{H}_{19} \mathrm{~N}_{2} \mathrm{~F}_{3} \mathrm{O}_{3}\right)\left[\mathrm{M}^{+}\right]$: calc. 356.1342, found 356.1341. IR (ATR, v): 3363, 2967, 1734, 1698, 1615, 1551, 1493, 1441, 1410, 1368, 1329, 1276, 1199, 1165, 1140, 1104, $1052,948,914,895,875,823,756,725,710,637,561,518,492,404 \mathrm{~cm}^{-1}$.

\section{2,2,2-Trifluoro-N-(3-(2-oxo-2H-4-chlorobenzo[b][1,4]oxazin-3-yl)propyl)acetamide (7ec)}<smiles>O=C(NCCCc1nc2cc(Cl)ccc2oc1=O)C(F)(F)F</smiles>

According to general procedure $3,300 \mathrm{mg}$ of resin $3 \mathbf{e}$ (batch $3 e .2,0.726 \mathrm{mmol} / \mathrm{g}, 0.218 \mathrm{mmol}$ ) were converted with $92 \mathrm{mg}$ of 2-amino-4-chlorophenol (640 mmol, 2.94 equiv.) in $4.0 \mathrm{~mL}$ of methanol to give $28.2 \mathrm{mg}(0.092 \mathrm{mmol})$ of the target substance in $42 \%$ yield as a deep yellow solid. $\mathrm{R}_{f}$ (cyclohexane/ethyl acetate, $2: 1$ ) $=0.48 .{ }^{1} \mathrm{H}$ NMR (500 MHz, CDCl, $\mathrm{ppm}$ ), $\delta=2.16$ (quin, $J=6.9 \mathrm{~Hz}, 2 \mathrm{H}$ ), $3.03(\mathrm{t}, J=7.0 \mathrm{~Hz}, 2 \mathrm{H}$ ), $3.52-3.56(\mathrm{~m}, 2 \mathrm{H}$ ), 6.85 (bs, $1 \mathrm{H}$ ), 7.28 (d, J=8.8 Hz, $1 \mathrm{H}$ ), 7.49 (dd, $J=8.8 \mathrm{~Hz}, 2.4 \mathrm{~Hz}, 1 \mathrm{H}$ ), 7.76 (d, $J=2.4 \mathrm{~Hz}, 1 \mathrm{H}$ ). ${ }^{13} \mathrm{C}$ $\operatorname{NMR}\left(125 \mathrm{MHz}, \mathrm{CDCl}_{3}, \mathrm{ppm}\right), \delta=24.6,31.0,39.4,115.8$ (q, $\left.J=286 \mathrm{~Hz}\right), 117.6,128.3,131.0,144.9$, $152.5,157.4$ (q, $J=38 \mathrm{~Hz}), 157.8 .{ }^{19} \mathrm{~F} \mathrm{NMR}\left(471 \mathrm{MHz}, \mathrm{CDCl}_{3}, \mathrm{ppm}\right), \delta=-75.85 . \mathrm{El}\left(\mathrm{m} / \mathrm{z}, 70 \mathrm{eV}, 90{ }^{\circ} \mathrm{C}\right)$ : 334/336 (48/17) [M] ${ }^{+}, 221 / 223$ (82/29), 208 (35), 195 (15), 180/182 (100/34), 167 (17). HRMS $\left(\mathrm{C}_{13} \mathrm{H}_{10} \mathrm{O}_{3} \mathrm{~N}_{2} \mathrm{ClF}_{3}\right)$ [M]: calc. 334.0327, found 334.0326. IR (ATR, ṽ): 3310, 2937, 1742, 1702, 1611, $1564,1481,1465,1415,1260,1206,1158,1096,1074,1047,1013,932,888,816,722,703,670$, $583,545,521,401 \mathrm{~cm}^{-1}$.

\section{3-(4-Hydroxyphenyl)-5-isopropyl-5,6-dihydro-2H-1,4-oxazin-2-one (7bf)}

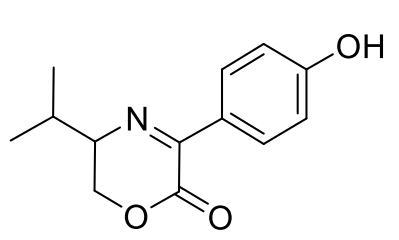

According to general procedure $3,300 \mathrm{mg}$ of resin $\mathbf{3 b}$ (batch $3 \mathrm{~b} .2$, $0.705 \mathrm{mmol} / \mathrm{g}, 0.212 \mathrm{mmol}$ ) were converted with $69.0 \mathrm{mg}$ of 2 -amino-3methylbutan-1-ol ( $0.669 \mathrm{mmol}, 3.16$ equiv.) in $4.0 \mathrm{~mL}$ of methanol to give $33.0 \mathrm{mg}(0.141 \mathrm{mmol})$ of the target substance in $67 \%$ yield as a light yellow solid. $\mathrm{R}_{f}$ (cyclohexane/ethyl acetate, $\left.1: 1\right)=0.15 .{ }^{1} \mathrm{H}$ NMR $(500 \mathrm{MHz}$, MeOD+CDCl $3, \mathrm{ppm}), \delta=0.95(\mathrm{~d}, J=6.9 \mathrm{~Hz}, 3 \mathrm{H}), 1.00(\mathrm{~d}, J=6.7 \mathrm{~Hz}, 3 \mathrm{H}), 1.90-1.99(\mathrm{~m}, 1 \mathrm{H}), 3.62-$ $3.71(m, 2 \mathrm{H}), 3.79-3.84(\mathrm{~m}, 1 \mathrm{H}), 6.82-6.87(\mathrm{~m}, 2 \mathrm{H}), 8.03-8.08(\mathrm{~m}, 2 \mathrm{H}) .{ }^{13} \mathrm{C} \mathrm{NMR}\left(125 \mathrm{MHz}, \mathrm{CDCl}_{3}\right.$, ppm), $\delta=19.0,20.1,29.8,57.7,62.8,116.3,125.8,134.2,164.6,166.6,188.9 . \mathrm{El}\left(\mathrm{m} / \mathrm{z}, 70 \mathrm{eV}, 90^{\circ} \mathrm{C}\right)$ : $233(61)[\mathrm{M}]^{+}, 160$ (55), 120 (100) .MS $\left(\mathrm{C}_{13} \mathrm{H}_{15} \mathrm{NO}_{3}\right.$ ) [M]: calc. 233.1046, found 233.1045. IR (ATR, v): 3324, 2961, 1796, 1724, 1650, 1578, 1513, 1439, 1367, 1277, 1196, 1164, 1034, 976, 843, 793, 731, $624,523 \mathrm{~cm}^{-1}$.

Polystyrenemethyl-3-(4-(4-(2-(tert-butoxy)-2-oxoethoxy)phenyl)-5-oxo-2-(trifluoromethyl)-2,5dihydrooxazol-2-yl)propanoate (9a).

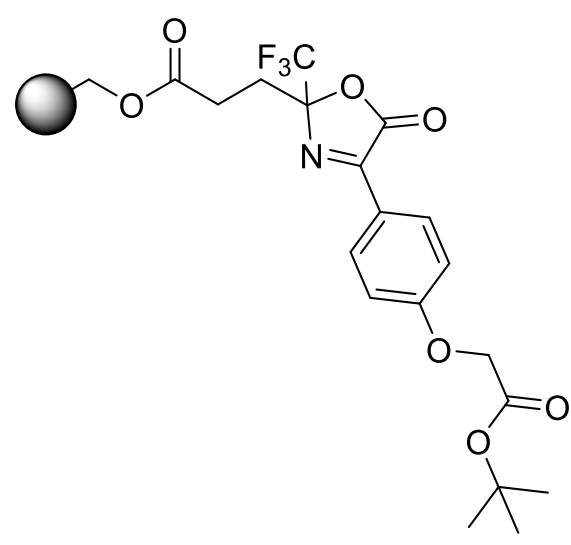

Resin 3b (batch 3b.3, $403 \mathrm{mg}$, loading: $0.649 \mathrm{mmol} / \mathrm{g}$, $0.261 \mathrm{mmol}$ ) was added to a solution of $\mathrm{K}_{2} \mathrm{CO}_{3}(95 \mathrm{mg}$, $138.2 \mathrm{~g} / \mathrm{mol}, 0.687 \mathrm{mmol}, 2.63$ equiv.) and tert-butyl 2bromoacetate $(0.1 \mathrm{~mL}, 132 \mathrm{mg}, 195 \mathrm{~g} / \mathrm{mol}, 0.677 \mathrm{mmol}, 2.59$ equiv.) in $5 \mathrm{~mL}$ of DMF. The mixture was shaken for $18 \mathrm{~h}$ at $60^{\circ} \mathrm{C}$. The resin was purified according to general washing procedure. The target resin was obtained after drying in high vacuum $(397.2 \mathrm{mg}$, loading $=0.657 \mathrm{mmol} / \mathrm{g})$. Comment: The obtained amount of resin was less than expected. This might be a result of impurities in a preliminary step that have been removed during the washing procedure. Another explanation is 
that some material may have been lost due to cleavage with dimethylamine present in the reaction mixture. The loading of the resin was calculated with $0.261 \mathrm{mmol}$ of functionalities (value according to the starting material). ${ }^{13} \mathrm{C} \mathrm{Gel-NMR}\left(75 \mathrm{MHz} \mathrm{CDCl}_{3}, \mathrm{ppm}\right), \delta=27.0,28.0,100.2,115.9,159.3 .{ }^{19} \mathrm{~F}$ NMR (376 MHz, CDCl 3 ppm), $\delta=-79.77$. IR (ṽ): 3024, 2921, 1799, 1736, 1640, 1597, 1514, 1492, $1450,1254,1142,1070,906,846,753,695,536 \mathrm{~cm}^{-1}$.

\section{Polystyrenemethyl-3-(5-oxo-4-(4-(prop-2-yn-1-yloxy)phenyl)-2-(trifluoromethyl)-2,5-} dihydrooxazol-2-yl)propanoate (9b).

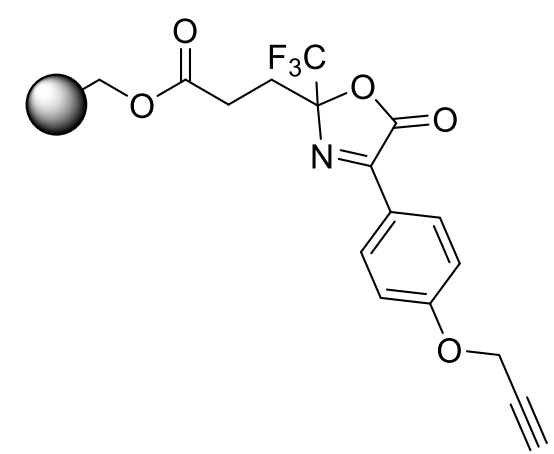

Resin 3b (batch 3b.2, $988.6 \mathrm{mg}$, loading: $0.705 \mathrm{mmol} / \mathrm{g}, 0.697$ $\mathrm{mmol}$ ) was added to a solution of $1.0 \mathrm{~mL}$ propargyl bromide and $\mathrm{K}_{2} \mathrm{CO}_{3}$ (600 mg, $4.34 \mathrm{mmol}, 6.22$ equiv.) in $20 \mathrm{~mL}$ of DMF. The mixture was shaken for $15 \mathrm{~h}$ at $\mathrm{rt}$. The resin was purified according to general washing procedure. After drying under high vacuum, $1.05 \mathrm{~g}$ (loading $=0.666$ ) of the target resin was obtained in quantitative yield. ${ }^{13} \mathrm{C}$ Gel-NMR $\left(100 \mathrm{MHz}, \mathrm{CDCl}_{3}\right.$, ppm), $\delta=26.9,55.8,66.9,100.4,115.3,120.6,125.7,131.3$, 145.2, 159.4, 162.0, 162.8, 170.9. ${ }^{19} \mathrm{~F}$ NMR (376 $\mathrm{MHz}, \mathrm{CDCl}_{3}$,

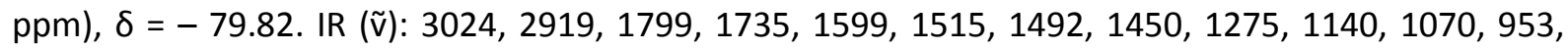
$844,754,696,534 \mathrm{~cm}^{-1}$.

Polystyrenemethyl-3-(5-oxo-4-(4-(prop-2-yn-1-yloxy)phenyl)-2-(trifluoromethyl)-2,5dihydrooxazol-2-yl)propanoate (10).

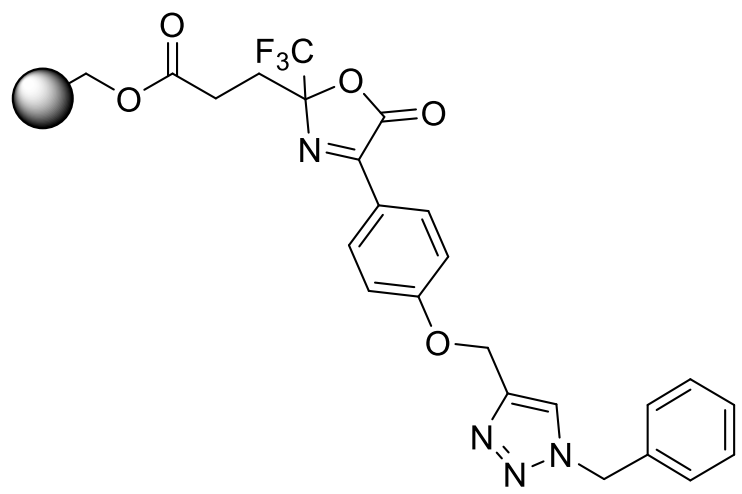

Resin 9b (311 mg, loading: $0.666 \mathrm{mmol} / \mathrm{g}$, $0.207 \mathrm{mmol}$ ) was reacted with Cul, sodium ascorbate and benzyl azide $(0.1 \mathrm{~mL}$; freshly prepared) in DCM for $2 \mathrm{~h}$ at $\mathrm{rt}$. The resulting resin was washed with acetone, cupral solution (until the filtrate was no longer black), then with methanol/DCM three times and DCM three times. After drying under high vacuum, the target resin was obtained in quantitative yield (339 $\mathrm{mg}$, loading: $0.611 \mathrm{mmol} / \mathrm{g}$ ). Raman spectrum shows no alkine specific peak. ${ }^{13} \mathrm{C}$ Gel-NMR (100 MHz, $\left.\mathrm{CDCl}_{3}, \mathrm{ppm}\right), \delta=26.9,54.2,62.1,66.9,115.2,120.2,122.8$, $128.0,128.8,131.2,134.1,143.4,159.2,162.6,171.2 .{ }^{19} \mathrm{~F} \mathrm{NMR}\left(376 \mathrm{MHz}, \mathrm{CDCl}_{3}, \mathrm{ppm}\right), \delta=-79.80 . \mathrm{IR}$ (ATR, ṽ): 3024, 2921, 1798, 1735, 1600, 1510, 1492, 1451, 1253, 1142, 1071, 953, 842, 754, 696, 536 $\mathrm{cm}^{-1}$.

tert-Butyl 2-(4-((4aR,8aR)-3-oxo-3,4,4a,5,6,7,8,8a-octahydroquinoxalin-2-yl)phenoxy)acetate (11a)

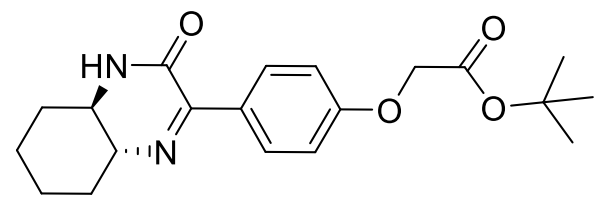

According to general procedure $3,334 \mathrm{mg}$ of resin $9 \mathrm{a}$ ( $0.657 \mathrm{mmol} / \mathrm{g}, 0.219 \mathrm{mmol}$ ) were converted with $107 \mathrm{mg}$ of cyclohexane-1,2-diamine $(0.937 \mathrm{mmol}, 4.28$ equiv. $)$ in $4.0 \mathrm{~mL}$ of methanol to give $23 \mathrm{mg}(64 \mu \mathrm{mol}, 358 \mathrm{~g} / \mathrm{mol})$ of the target substance in $29 \%$ yield as a brown solid. $\mathrm{R}_{f}\left(\mathrm{CH}_{2} \mathrm{Cl}_{2} / \mathrm{MeOH}, 30: 1\right)=0.34 .{ }^{1} \mathrm{H}$ NMR $(400 \mathrm{MHz}$, $\left.\mathrm{CDCl}_{3}, \mathrm{ppm}\right), \delta={ }^{1} \mathrm{H} \mathrm{NMR}\left(400 \mathrm{MHz}, \mathrm{CDCl}_{3}, \mathrm{ppm}\right), \delta=1.44-1.53(\mathrm{~m}, 13 \mathrm{H}), 1.78-2.00(\mathrm{~m}, 3 \mathrm{H}), 2.39-$ $2.46(\mathrm{~m}, 1 \mathrm{H}), 3.16-3.23(\mathrm{~m}, 2 \mathrm{H}), 4.54(\mathrm{~s}, 2 \mathrm{H}), 6.40(\mathrm{~s}, 1 \mathrm{H}), 6.87-6.93(\mathrm{~m}, 2 \mathrm{H}), 7.91-7.99(\mathrm{~m}, 2 \mathrm{H})$. ${ }^{13} \mathrm{C} \mathrm{NMR}\left(100 \mathrm{MHz}, \mathrm{CDCl}_{3}, \mathrm{ppm}\right), \delta=23.7,25.2,28.0,31.0,31.9,54.0,62.9,65.6,82.5,114.1,128.5$, 
130.6, 158.3, 159.7, 160.2, 167.7. El (m/z, $\left.70 \mathrm{eV}, 140{ }^{\circ} \mathrm{C}\right): 358$ (100) [M] $]^{+}, 302$ (47), 274 (38), 275 (15), 231 (32), 178 (36). HRMS $\left(\mathrm{C}_{20} \mathrm{H}_{26} \mathrm{~N}_{2} \mathrm{O}_{4}\right)[\mathrm{M}+\mathrm{H}]$ : calc. 358.1887, found 358.1885. IR (ATR, v): 3238, 2923, 2855, 1697, 1674, 1603, 1589, 1565, 1508, 1444, 1365, 1329, 1311, 1281, 1258, 1229, 1177, $1154,1072,940,839,795,779,712,655,594,575,520,486,441,408 \mathrm{~cm}^{-1}$.

(4aR,8aR)-3-(4-(prop-2-yn-1-yloxy)phenyl)-4a,5,6,7,8,8a-hexahydroquinoxalin-2(1H)-one (11b)

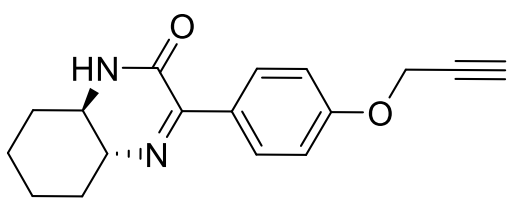
According to general procedure $3,93 \mathrm{mg}$ of resin $9 \mathbf{b}$ $(0.666 \mathrm{mmol} / \mathrm{g}, 0.136 \mathrm{mmol})$ were converted with $33.6 \mathrm{mg}$ of cyclohexane-1,2-diamine $(0.292 \mathrm{mmol}, 2.15$ equiv.) in $4.0 \mathrm{~mL}$ of methanol to give $14 \mathrm{mg}(49.6 \mu \mathrm{mol}, 282.3 \mathrm{~g} / \mathrm{mol})$ of the target substance in $36 \%$ yield as a brown solid. $\mathrm{R}_{f}$ (cyclohexane/ethyl acetate, $1: 1)=0.40 .{ }^{1} \mathrm{H}$ NMR $\left(500 \mathrm{MHz}, \mathrm{CDCl}_{3}, \mathrm{ppm}\right), \delta=1.33-1.56(\mathrm{~m}, 4 \mathrm{H}), 1.81-2.00(\mathrm{~m}, 3 \mathrm{H})$, $2.43(\mathrm{~d}, J=11.0 \mathrm{~Hz}, 1 \mathrm{H}), 2.52(\mathrm{t}, J=2.4 \mathrm{~Hz}, 1 \mathrm{H}), 3.19-3.24(\mathrm{~m}, 2 \mathrm{H}), 4.73(\mathrm{~d}, J=2.2 \mathrm{~Hz}, 2 \mathrm{H}), 6.04(\mathrm{br}$. s., $1 \mathrm{H}), 6.96-7.01(\mathrm{~m}, 2 \mathrm{H}), 7.92-7.97(\mathrm{~m}, 2 \mathrm{H}) .{ }^{13} \mathrm{C} \mathrm{NMR}\left(125 \mathrm{MHz}, \mathrm{CDCl}_{3}, \mathrm{ppm}\right), \delta=23.7,25.2$, 31.1, 31.9, 54.0, 55.8, 62.9, 75.7, 78.2, 114.4, 128.5, 130.6, 158.1, 159.3, 160.3. El (m/z, 70 eV, 130 $\left.{ }^{\circ} \mathrm{C}\right): 282(100)[\mathrm{M}]^{+}, 253 / 254$ (18/14), 215 (40), $199(16), 158(46)$. HRMS $\left(\mathrm{C}_{17} \mathrm{H}_{18} \mathrm{~N}_{2} \mathrm{O}_{2}\right)\left[\mathrm{M}^{+}\right]$: calc. 282.1363, found 282.1364. IR (ATR, ṽ): 3267, 2923, 2858, 2121, 1675, 1587, 1560, 1506, 1447, 1402, $1361,1297,1260,1224,1176,1067,1023,989$ 929, 869, 835, 775, 711, 675, 649, 623, 574, 540, 518, $484,464,412 \mathrm{~cm}^{-1}$.

(4aR,8aR)-3-(4-((1-benzyl-1H-1,2,3-triazol-4-yl)methoxy)phenyl)-4a,5,6,7,8,8ahexahydroquinoxalin-2(1H)-one (11c)

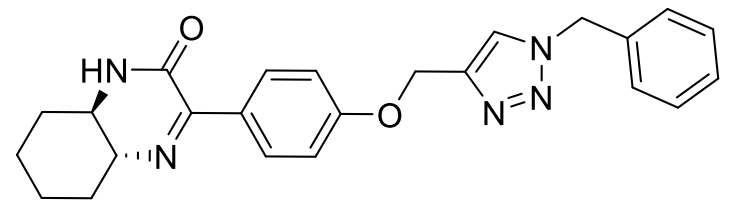

According to general procedure 3, $207 \mathrm{mg}$ of resin $10(0.611 \mathrm{mmol} / \mathrm{g}, 0.126 \mathrm{mmol})$ were converted with $65.6 \mathrm{mg}$ of cyclohexane-1,2-diamine $(0.575$ mmol, 4.56 equiv.) in $4.0 \mathrm{~mL}$ of methanol to give

$18.7 \mathrm{mg}(45.1 \mu \mathrm{mol}, 415 \mathrm{~g} / \mathrm{mol})$ of the target substance in $36 \%$ yield as a brown solid. $\mathrm{R}_{f}$ $\left(\mathrm{CH}_{2} \mathrm{Cl}_{2} / \mathrm{MeOH}, 30: 1\right)=0.36 .{ }^{1} \mathrm{H}$ NMR $\left(400 \mathrm{MHz}, \mathrm{CDCl}_{3}, \mathrm{ppm}\right), \delta=1.23-1.53(\mathrm{~m}, 4 \mathrm{H}), 1.77-2.00(\mathrm{~m}$, $3 \mathrm{H}), 2.41(\mathrm{~d}, J=10.4 \mathrm{~Hz}, 1 \mathrm{H}$ ), $3.18(\mathrm{dd}, J=6.6 \mathrm{~Hz}, J=2.5 \mathrm{~Hz}, 2 \mathrm{H}), 5.22(\mathrm{~s}, 2 \mathrm{H}), 5.52(\mathrm{~s}, 2 \mathrm{H}), 6.66$ (bs, $1 \mathrm{H}), 6.95-6.97(\mathrm{~m}, 2 \mathrm{H}), 7.26-7.30(\mathrm{~m}, 2 \mathrm{H}), 7.34-7.41(\mathrm{~m}, 3 \mathrm{H}), 7.52(\mathrm{~s}, 1 \mathrm{H}), 7.90-7.92(\mathrm{~m}, 2 \mathrm{H})$. ${ }^{13} \mathrm{C} \mathrm{NMR}\left(100 \mathrm{MHz}, \mathrm{CDCl}_{3}, \mathrm{ppm}\right), \delta=23.6,25.1,30.9,31.8,53.9,54.2,62.0,62.8,114.2,122.6,128.1$, $128.2,128.8,129.1,130.6,134.4,144.3,158.4,159.9,160.3$. El (m/z, $\left.70 \mathrm{eV}, 220^{\circ} \mathrm{C}\right): 415$ (4) [M]+, 244 (8), 173 (7), 144 (27), 84 (100). HRMS $\left(\mathrm{C}_{24} \mathrm{H}_{25} \mathrm{~N}_{5} \mathrm{O}_{2}\right)$ [M+H]: calc. 415.2003, found 415.2004. IR (ATR, ṽ): 3179, 3081, 2922, 2857, 2228, 1677, 1591, 1568, 1508, 1458, 1360, 1309, 1243, 1218, 1778, $1221,1072,1052,1004,988,921,839,765,717,641,623,575,535,487,463,398 \mathrm{~cm}^{-1}$.

\section{Crystallographic Data}

\section{Crystal Structure Determinations}

The single-crystal X-ray diffraction study was carried out on a Bruker-Nonius KappaCCD diffractometer at 123(2) K using Mo-Ka (7ba-SB214_HY) and a Bruker D8 Venture diffractometer with Photon 100 detector at 123(2) K using Cu-K $\alpha$ (7bb-SB808_HY) $(\lambda=$ $0.71073 \AA$ and $1.54178 \AA$ ). Direct Methods (SHELXS-97) ${ }^{\mathrm{a}}$ were used for structure solution and refinement was carried out using SHELXL-98 $\%$ SHELXL-2014 ${ }^{\mathrm{b}}$ (full-matrix least-squares on $F^{2}$ ). Hydrogen atoms were localized by difference electron density determination and refined using a riding model $(\mathrm{H}(\mathrm{O})$ free). A semi-empirical absorption 
corrections was applied for 7bb-SB808_HY. For 7be-SB808_HY an extinction correction was applied.

7ba-SB214_HY: colourless crystals, $\mathrm{C}_{14} \mathrm{H}_{9} \mathrm{NO}_{3}, M_{\mathrm{r}}=239.22$, crystal size $0.24 \times 0.06 \times$ $0.04 \mathrm{~mm}$, monoclinic, space group $P 2_{1} / \mathrm{n}$ (No. 14), $a=6.162(1) \AA, b=7.542(1) \AA, c=$ $22.825(3) \AA, \beta=90.28(1)^{\circ}, V=1060.8(3) \AA^{3}, Z=4, \rho=1.498 \mathrm{Mg} / \mathrm{m}^{-3}, \mu\left(\mathrm{Mo}-\mathrm{K}_{\alpha}\right)=0.107$ $\mathrm{mm}^{-1}, F(000)=496,2 \theta_{\max }=50.0^{\circ}, 8566$ reflections, of which 1872 were independent $\left(R_{\text {int }}\right.$ $=0.085$ ), 166 parameters, 1 restraint, $R_{1}=0.042$ (for $1467 \mathrm{I}>2 \sigma(\mathrm{I})$ ), $\mathrm{w} R_{2}=0.111$ (all data), $S=1.04$, largest diff. peak $/$ hole $=0.223 /-0.234 \mathrm{e}^{-3}$.

7be-SB808_HY: colourless crystals, $\mathrm{C}_{15} \mathrm{H}_{1} \mathrm{NO}_{3}, M_{\mathrm{r}}=253.25$, crystal size $0.36 \times 0.18 \times$ $0.10 \mathrm{~mm}$, monoclinic, space group $P 2_{1} / \mathrm{n}$ (No. 14), $a=6.2023(3) \AA, b=23.1025(12) \AA, c$ $=8.3582(4) \AA, \beta=99.204(1)^{\circ}, V=1182.22(10) \AA^{3}, Z=4, \rho=1.423 \mathrm{Mg} / \mathrm{m}^{-3}, \mu\left(\mathrm{Cu}-\mathrm{K}_{\alpha}\right)=$ $0.826 \mathrm{~mm}^{-1}, F(000)=528,2 \theta_{\max }=144.2^{\circ}, 24888$ reflections, of which 2325 were independent $\left(R_{\mathrm{int}}=0.023\right), 177$ parameters, 1 restraint, $R_{1}=0.032$ (for $2287 \mathrm{I}>2 \sigma(\mathrm{I})$ ), $\mathrm{w} R_{2}=0.087$ (all data), $S=1.06$, largest diff. peak $/$ hole $=0.308 /-0.176 \mathrm{e}^{-3}$.

CCDC 1442205 (7ba-SB214_HY), and 1442198 (7be-SB808_HY) contain the supplementary crystallographic data for this paper. These data can be obtained free of charge from The Cambridge Crystallographic Data Centre via www.ccdc.cam.ac.uk/data request/cif.

a) G. M. Sheldrick, Acta Crystallogr. 2008, A64, 112-122; b) G. M. Sheldrick, Acta Crystallogr. 2015, C71, 3-8.

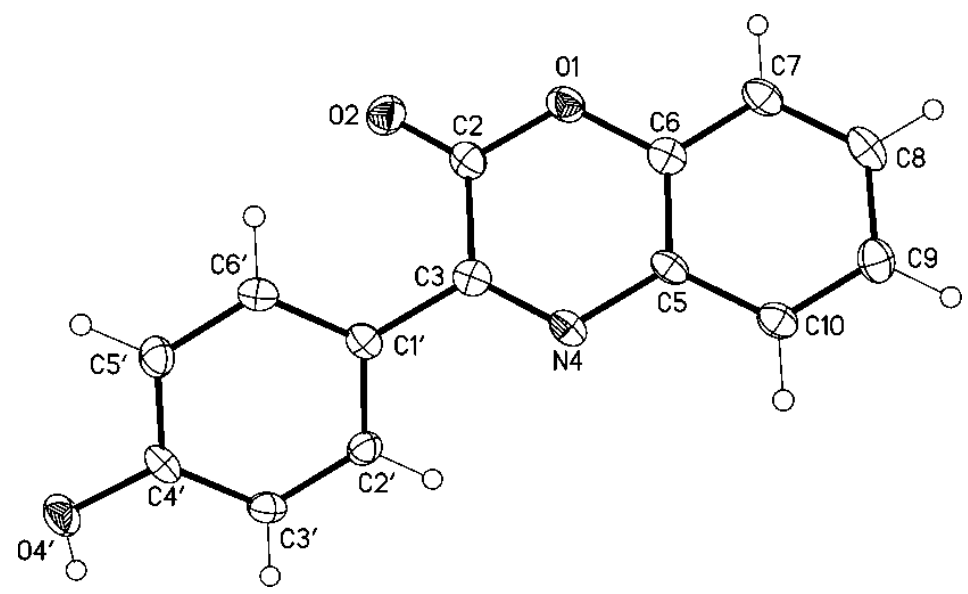

Fig. S1. Molecular structure of 7ba_SB214_HY (displacement parameters are drawn at $50 \%$ probability level). 


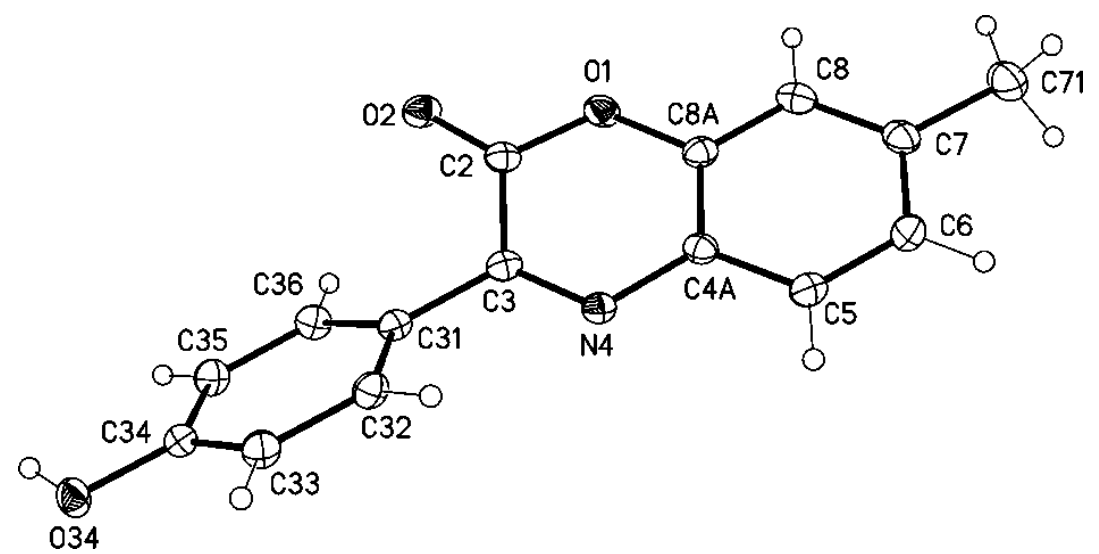

Fig. S2. Molecular structure of 7be-SB808_HY (displacement parameters are drawn at $50 \%$ probability level). 
Spectra

Compound 2 a
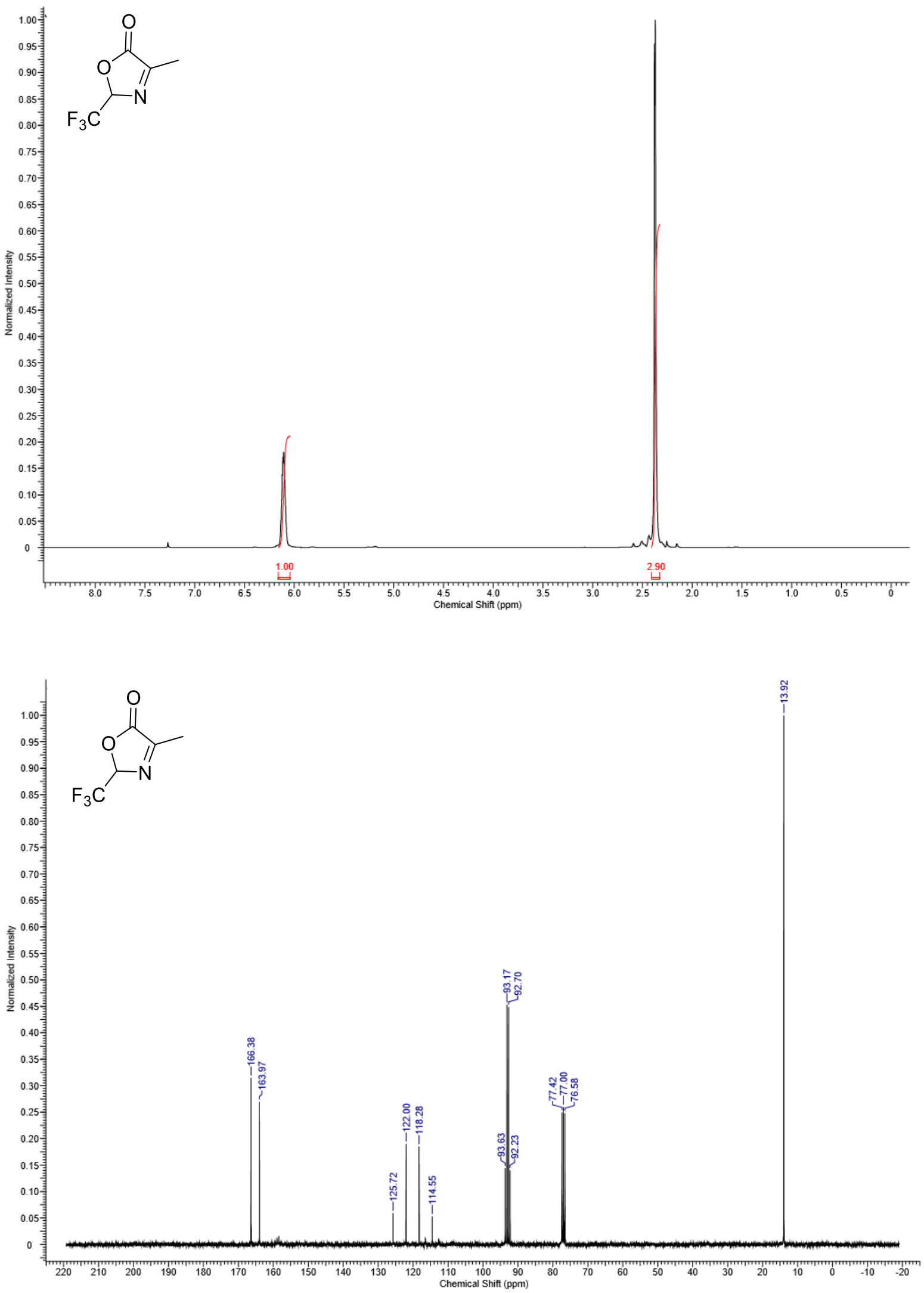
Compound 2b
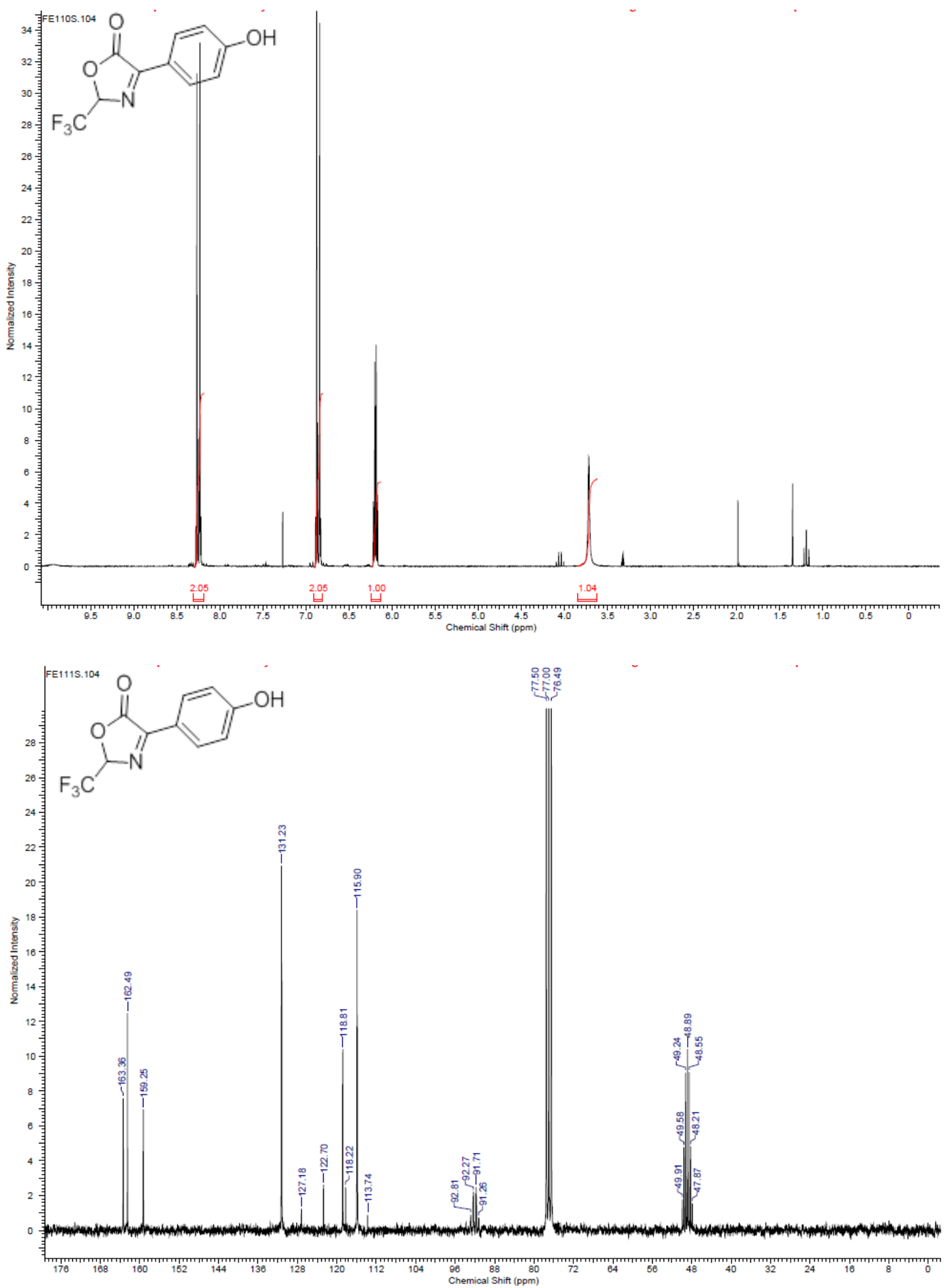
Compound ac
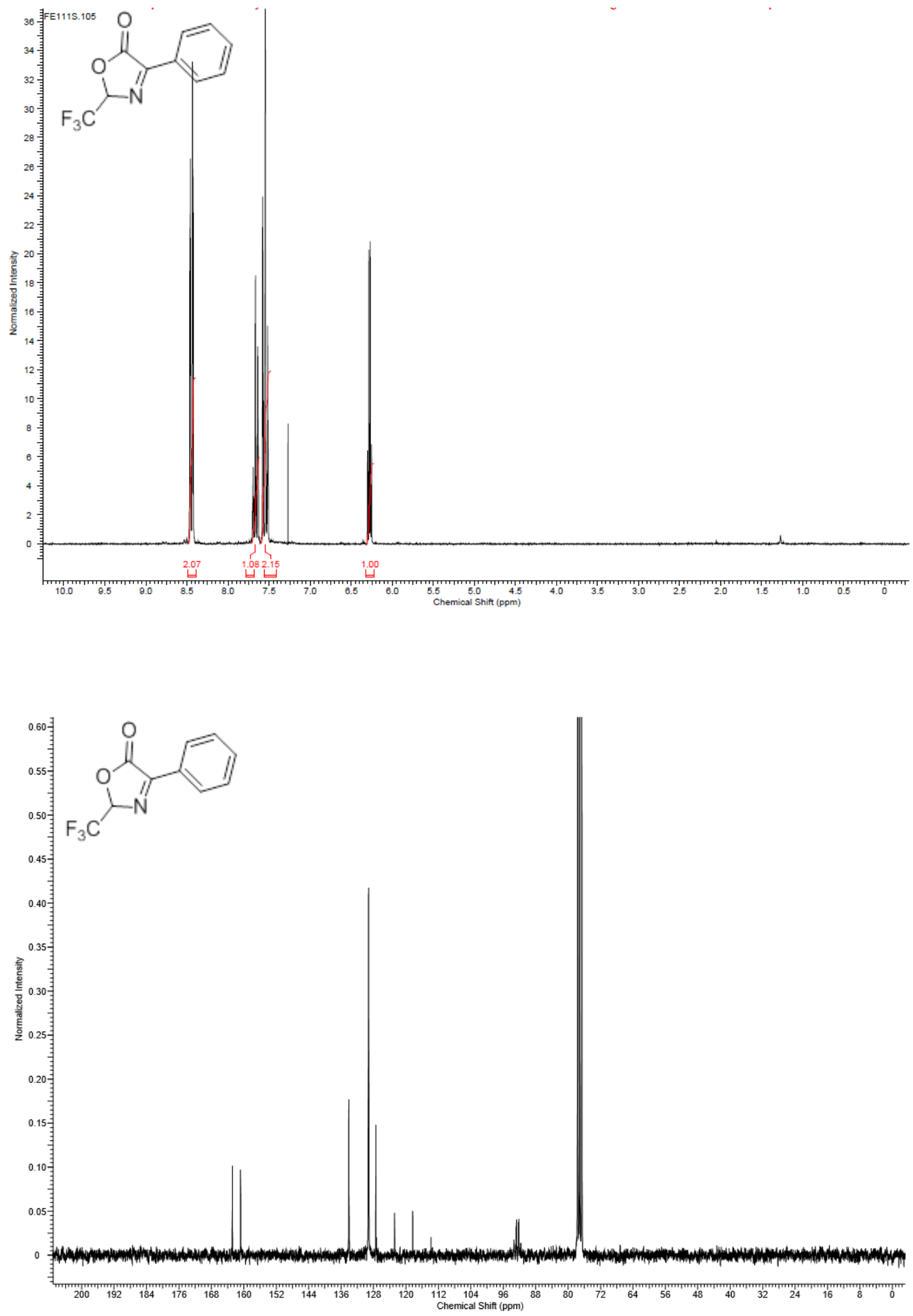


\section{Compound 2d}
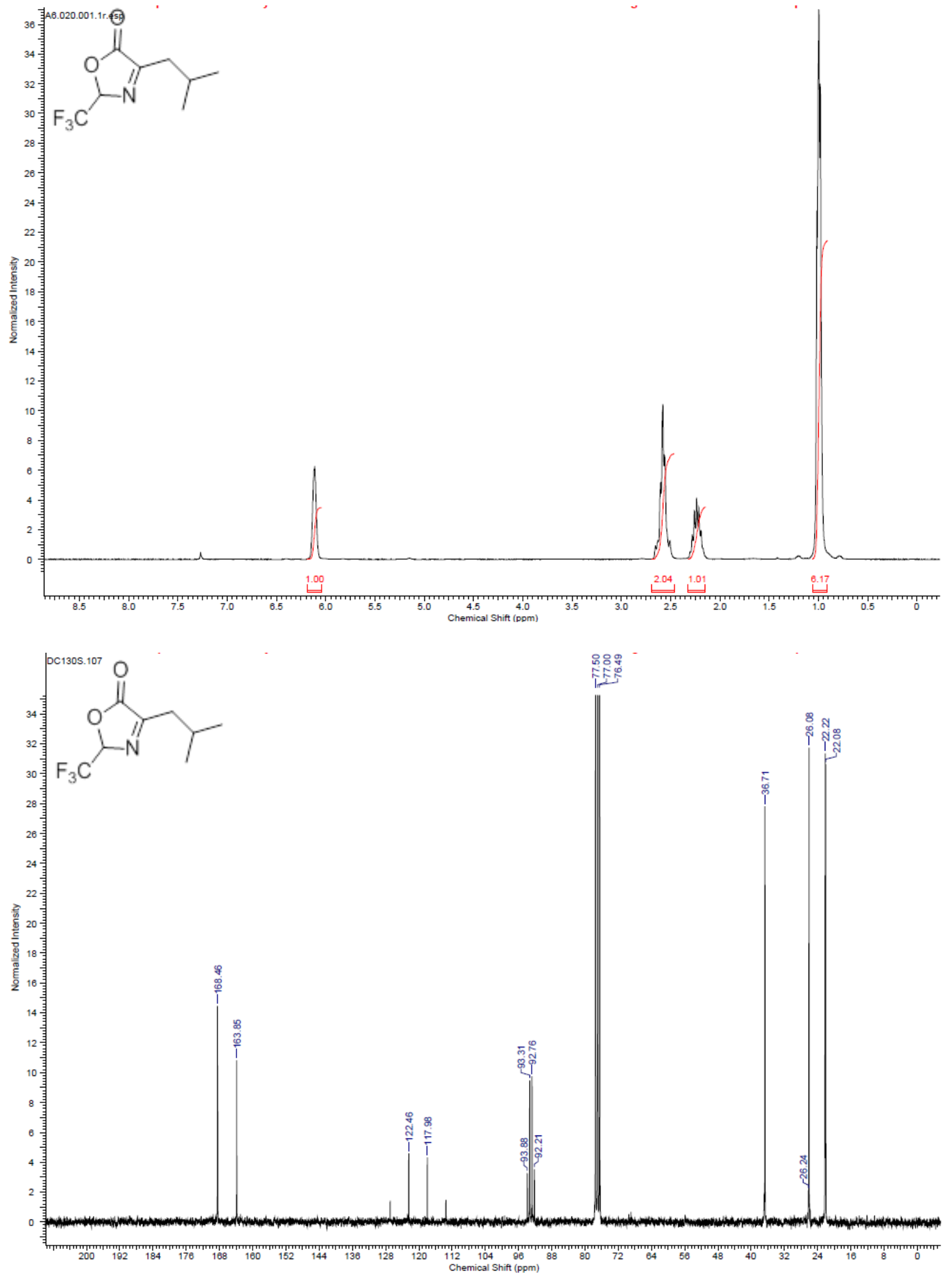


\section{Compound $2 \mathrm{e}$}
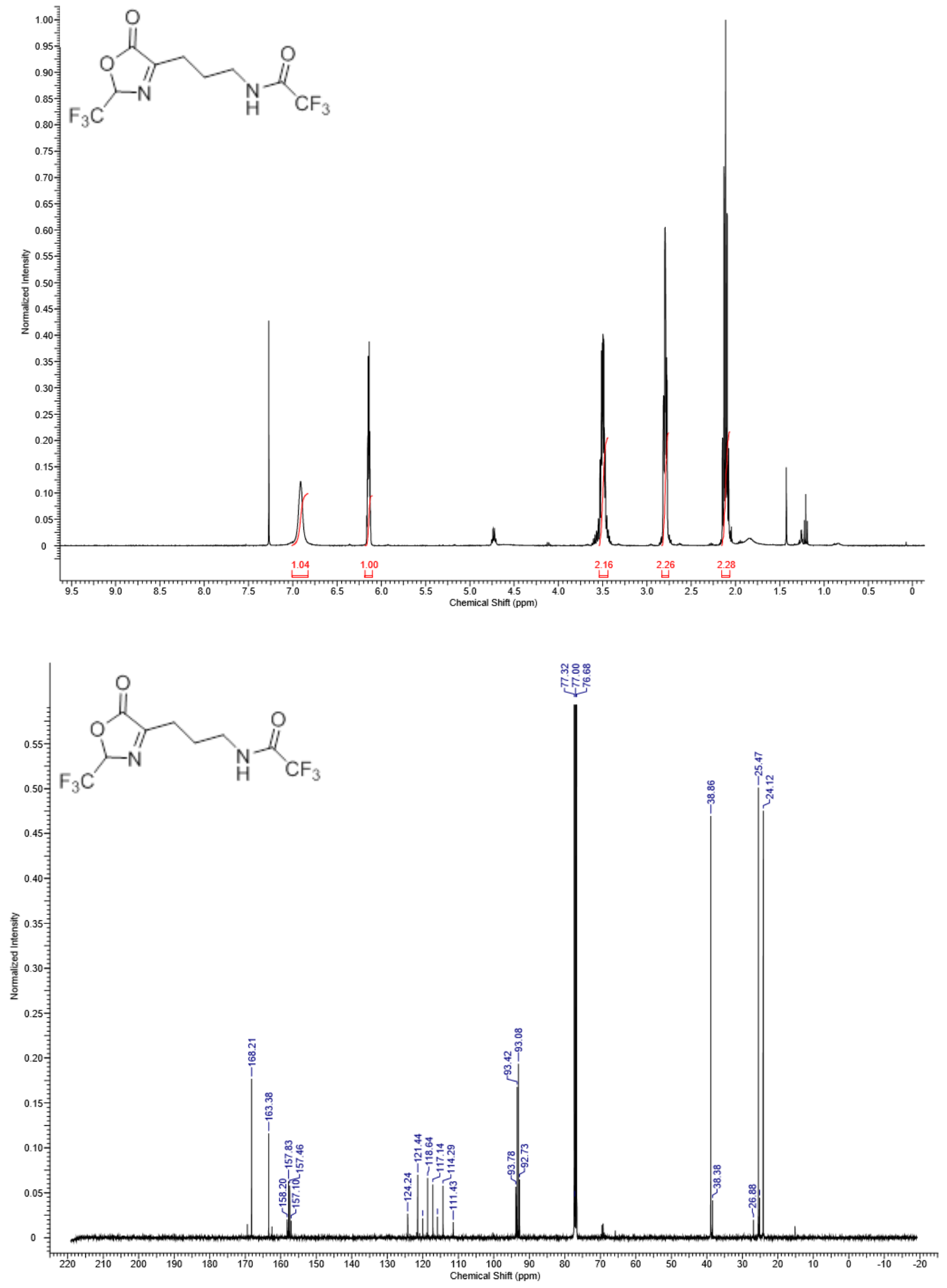


\section{Resin 3a}

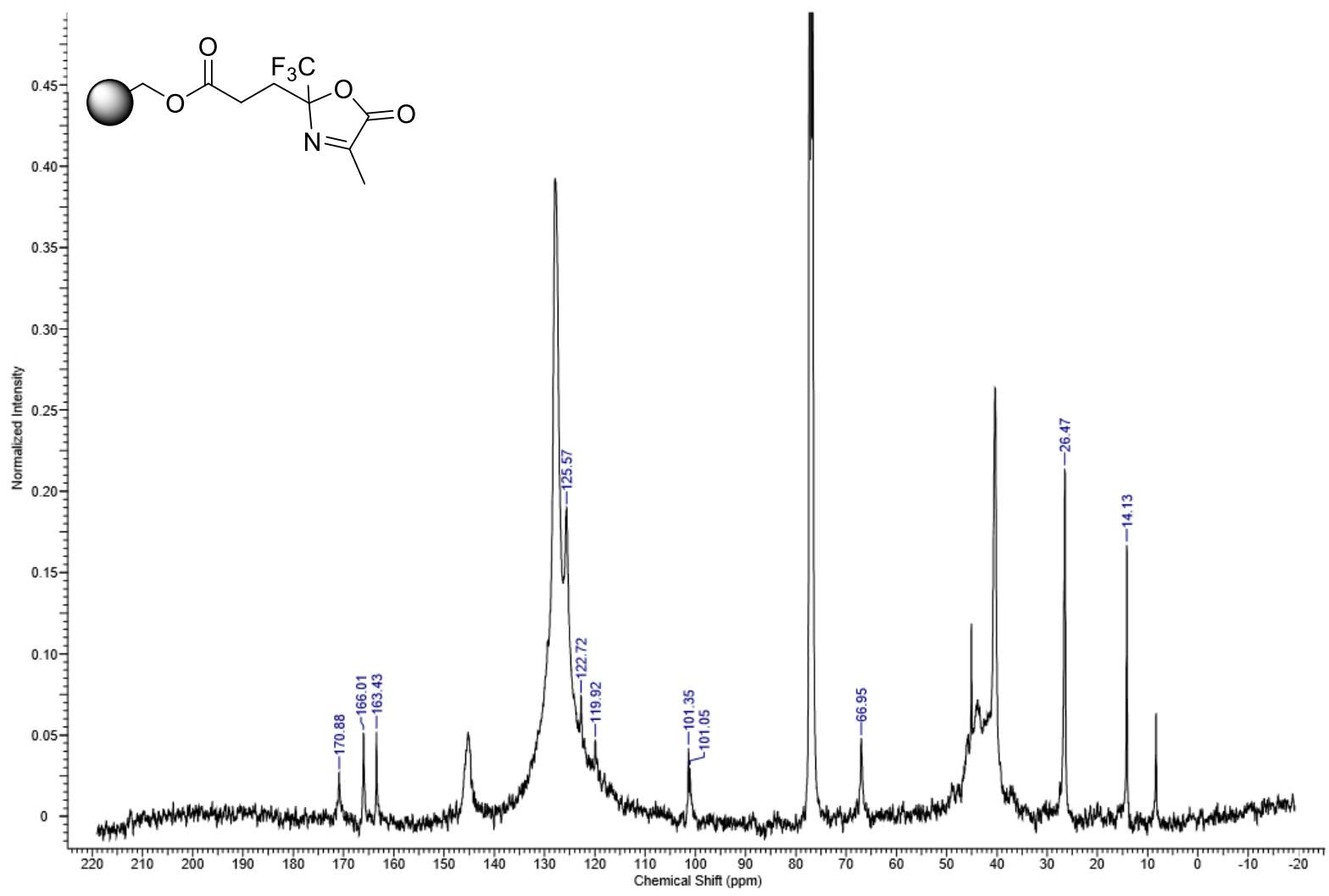

Resin $\mathbf{3 b}$

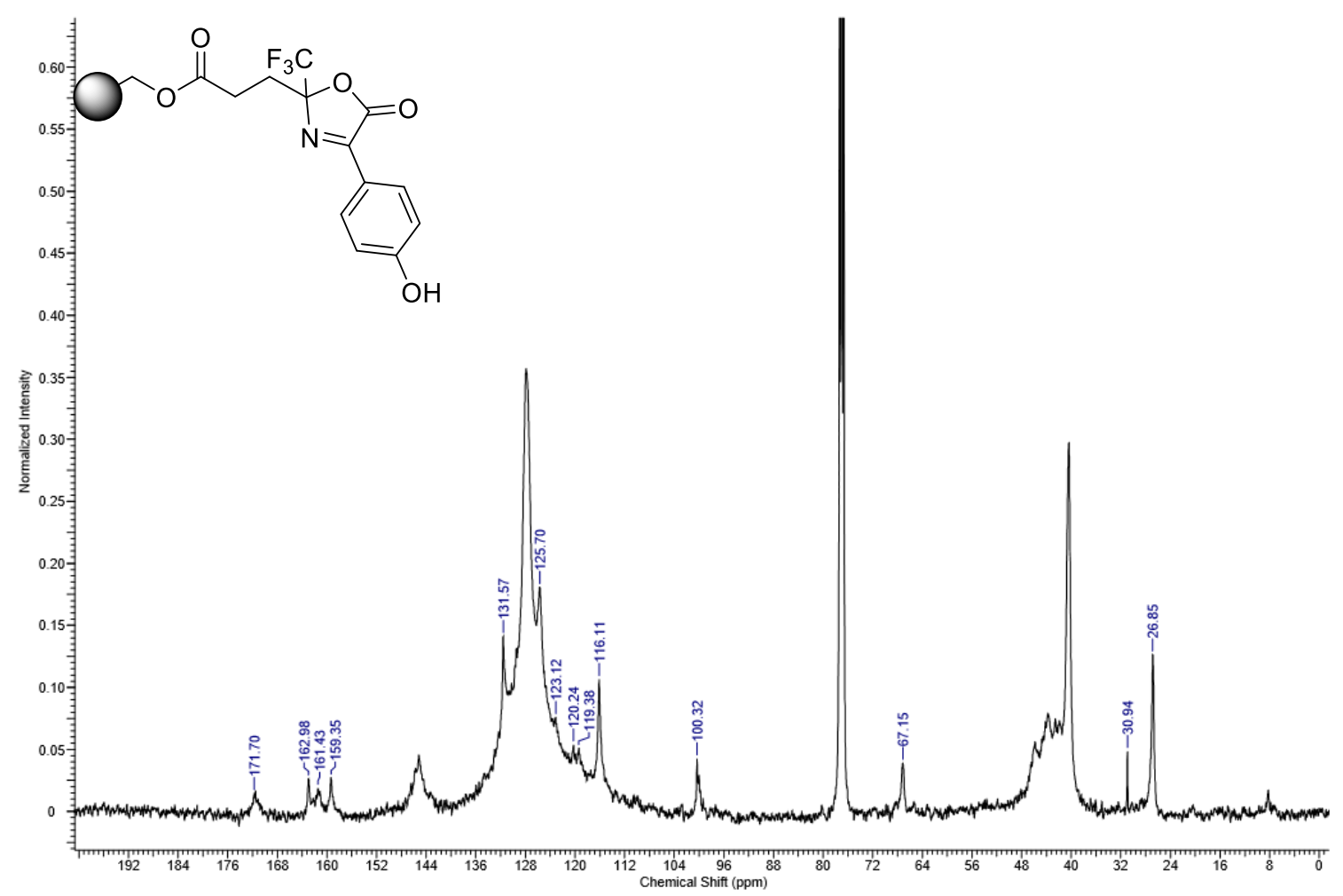




\section{Resin 3c}

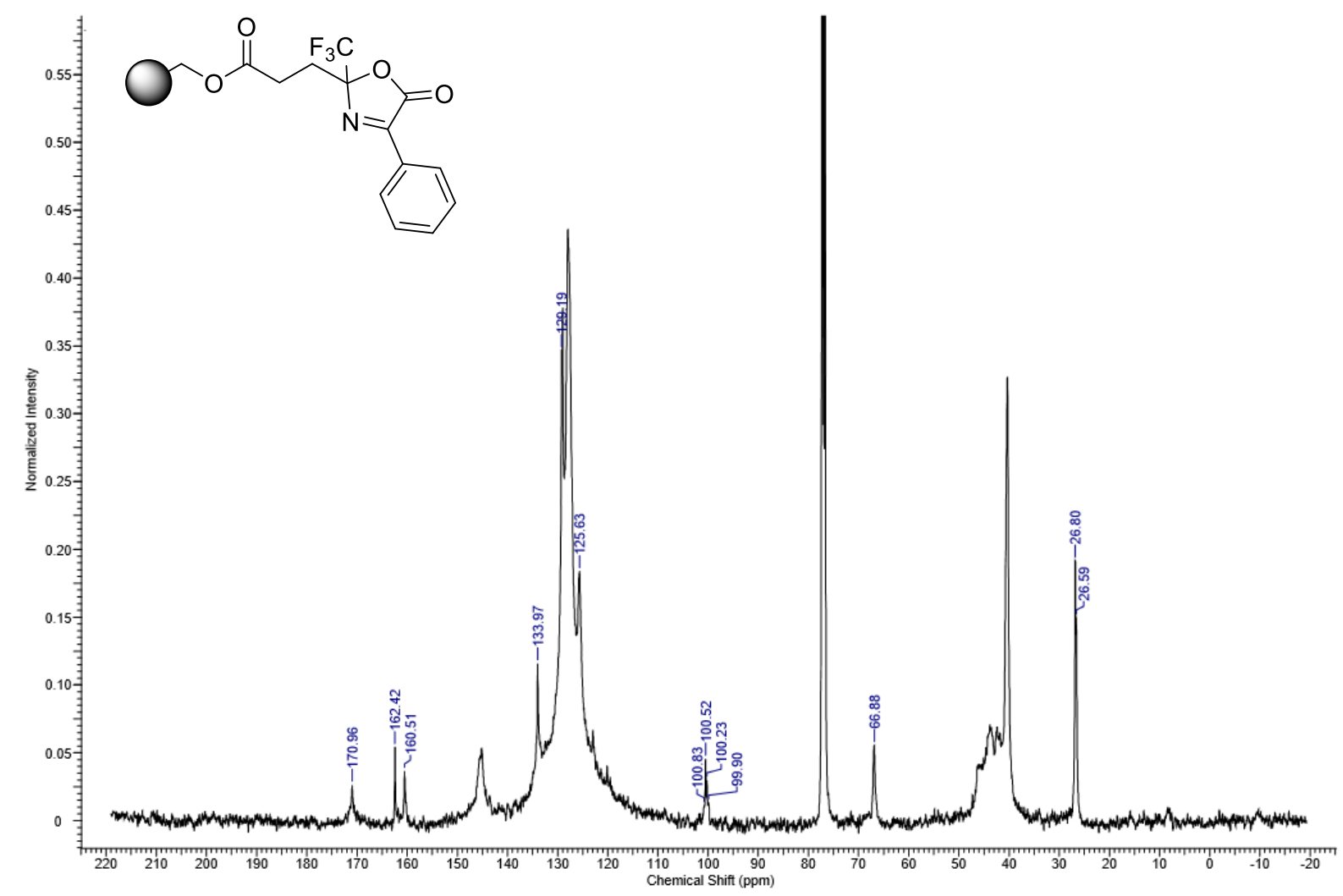

Resin 3d

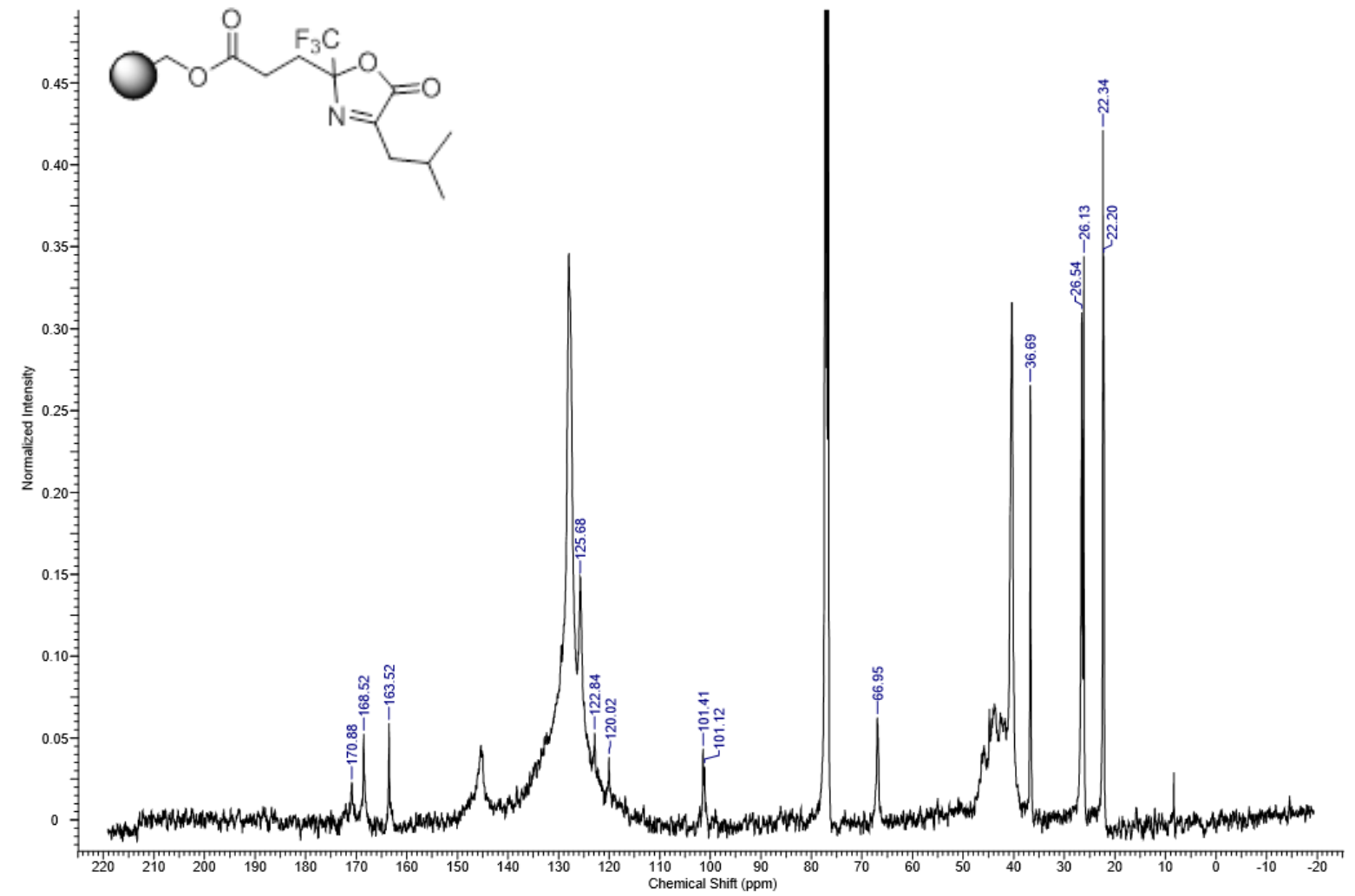




\section{Resin 3e}

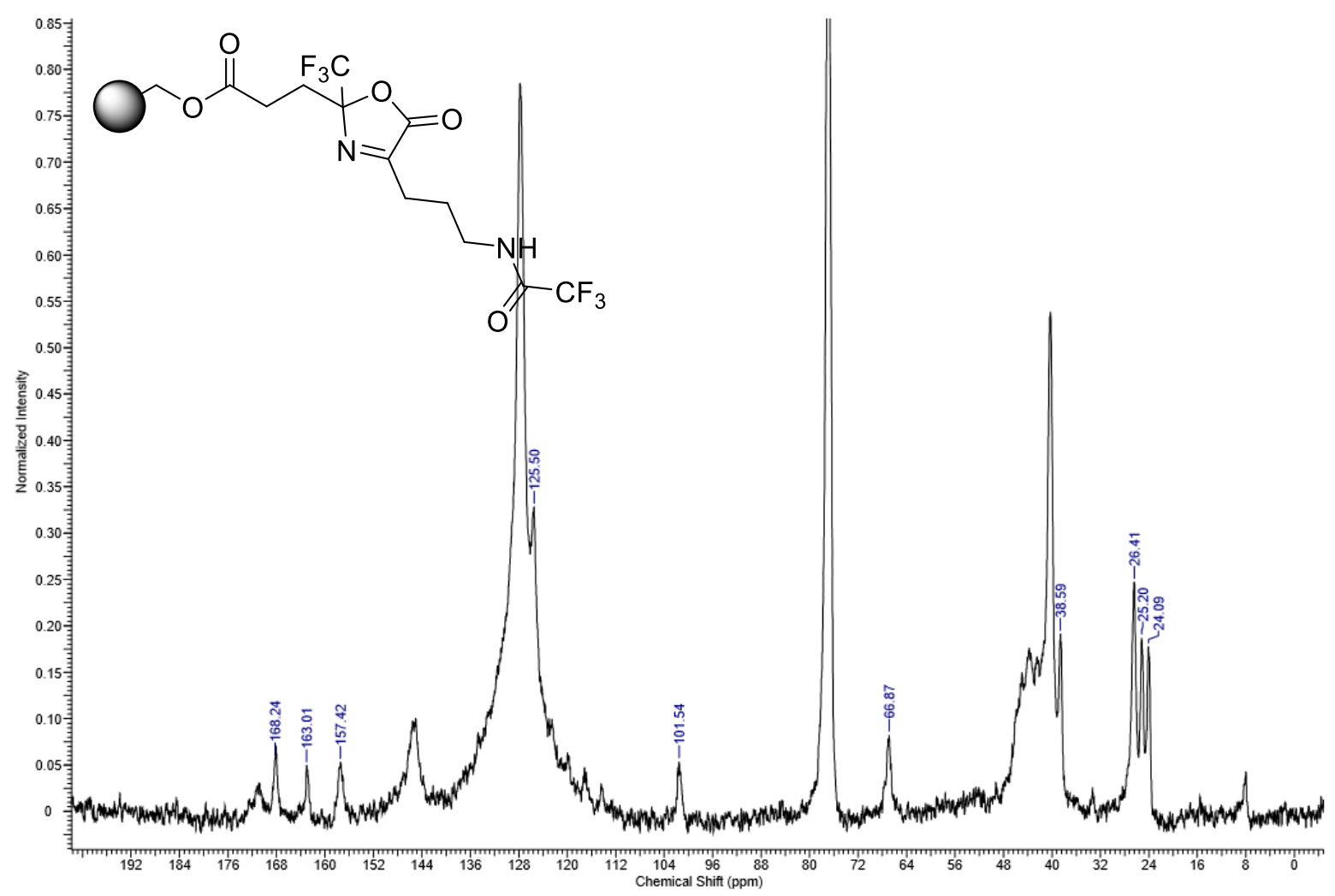




\section{Compound 5ba}
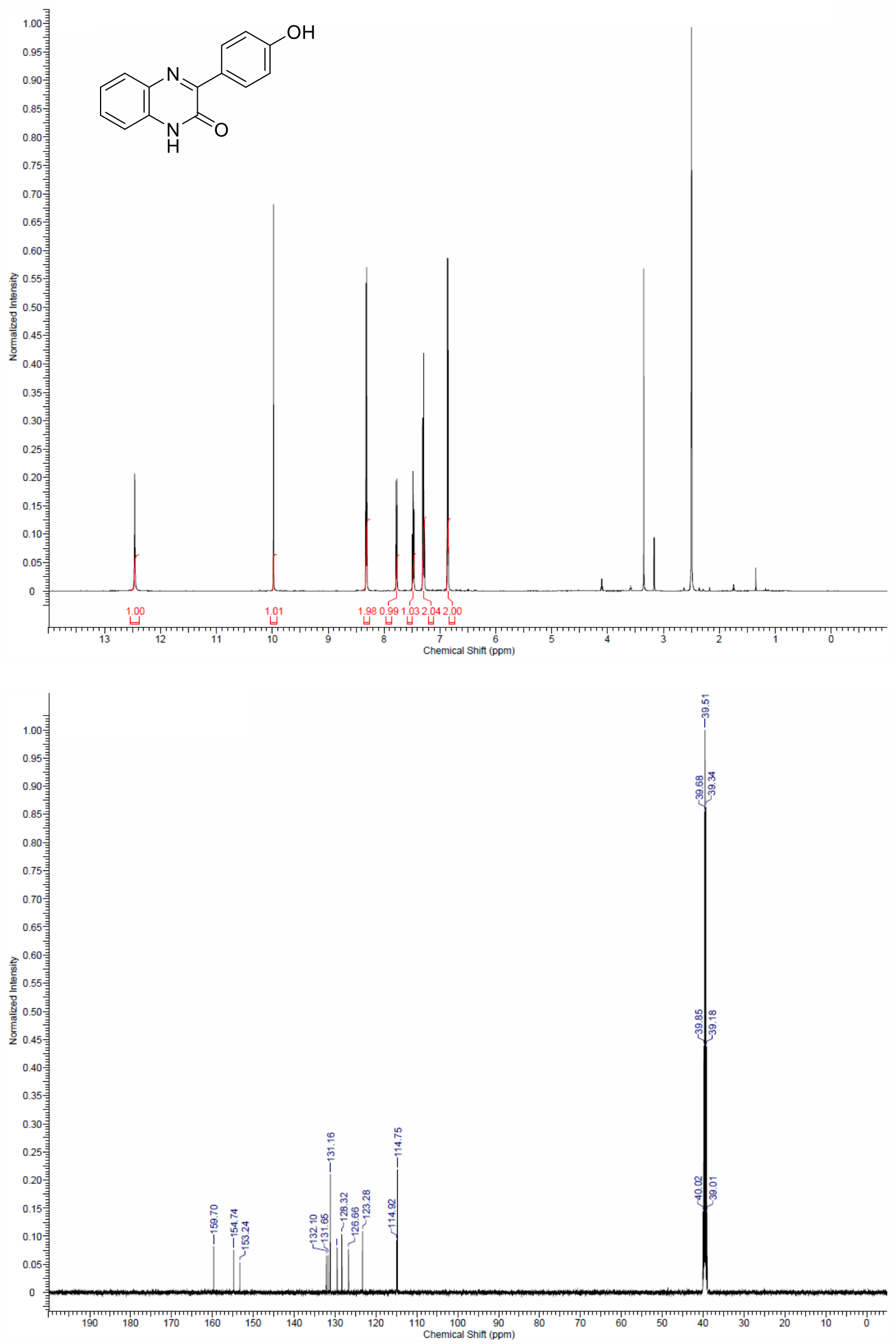


\section{Compound 5ca}
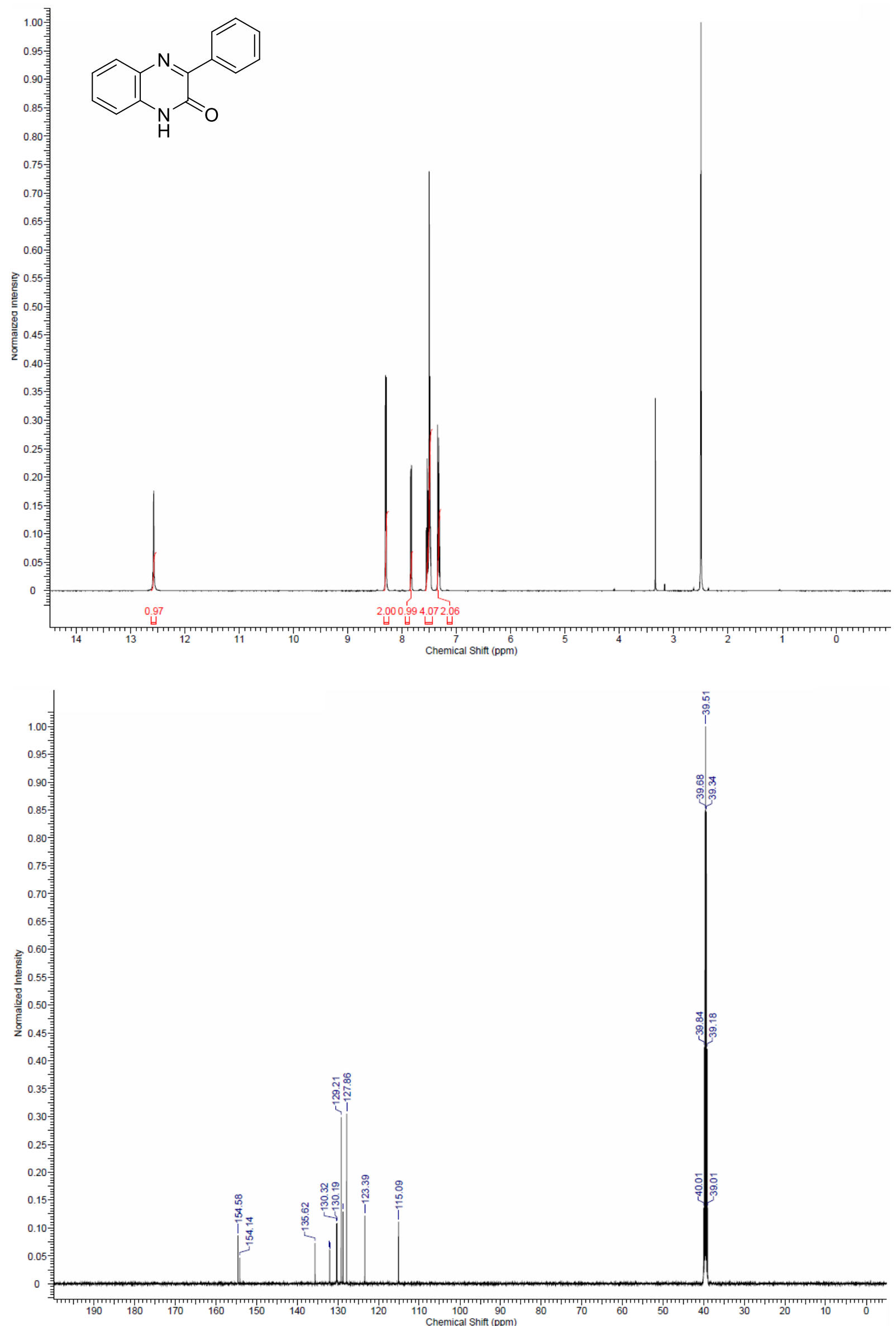
Compound 5cb
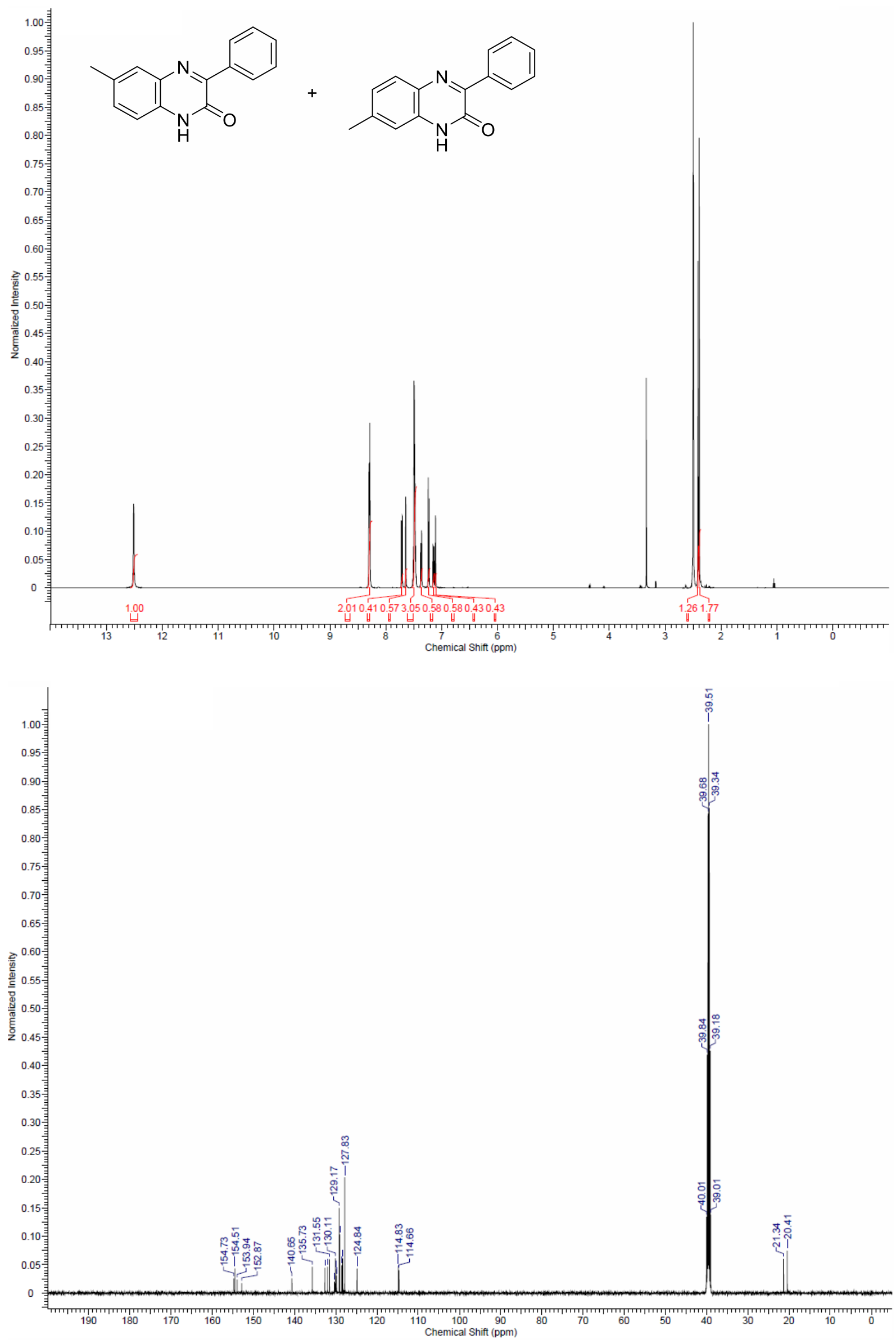


\section{Compound 5da}
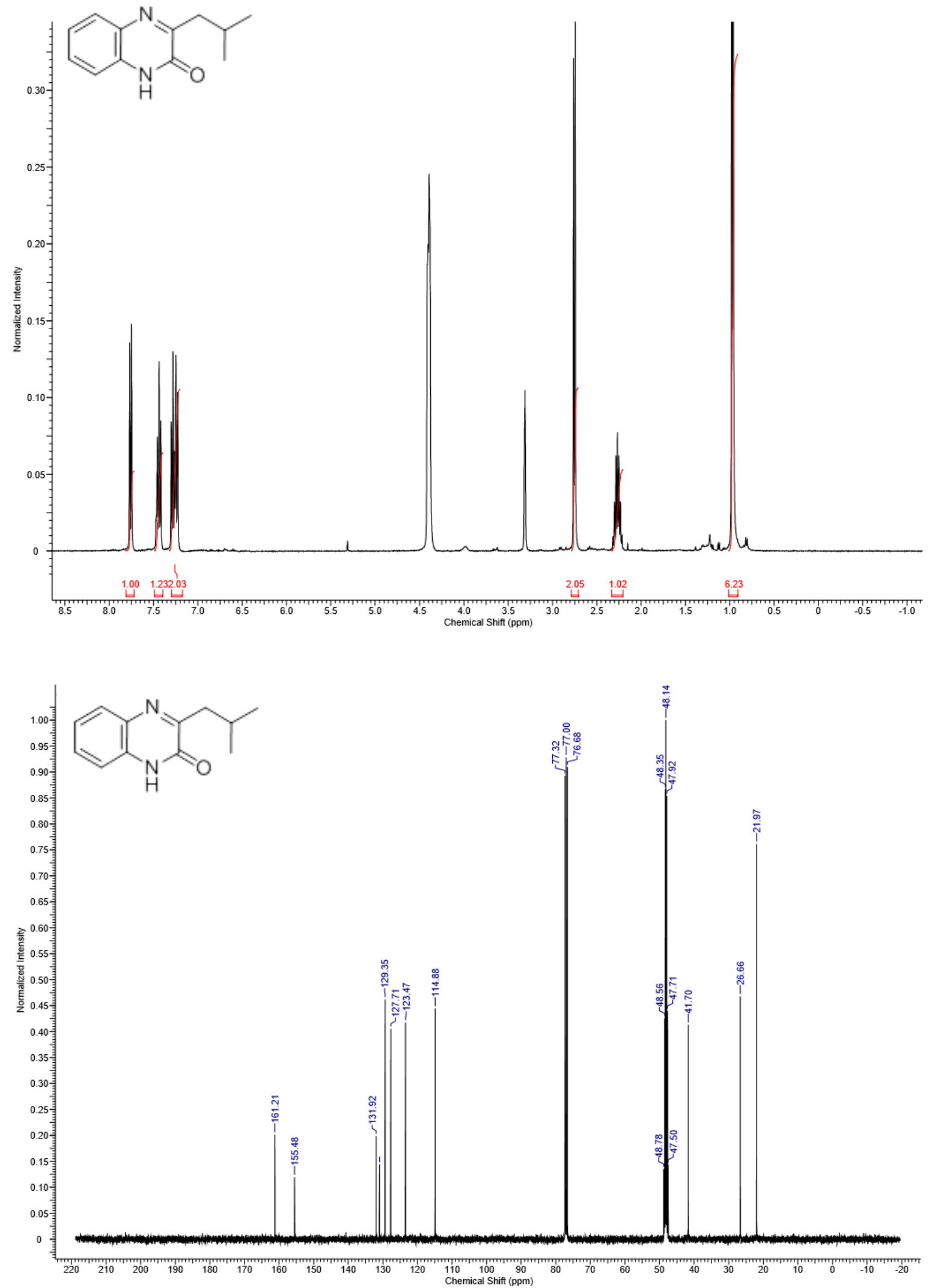


\section{Compound $5 \mathrm{db}$}
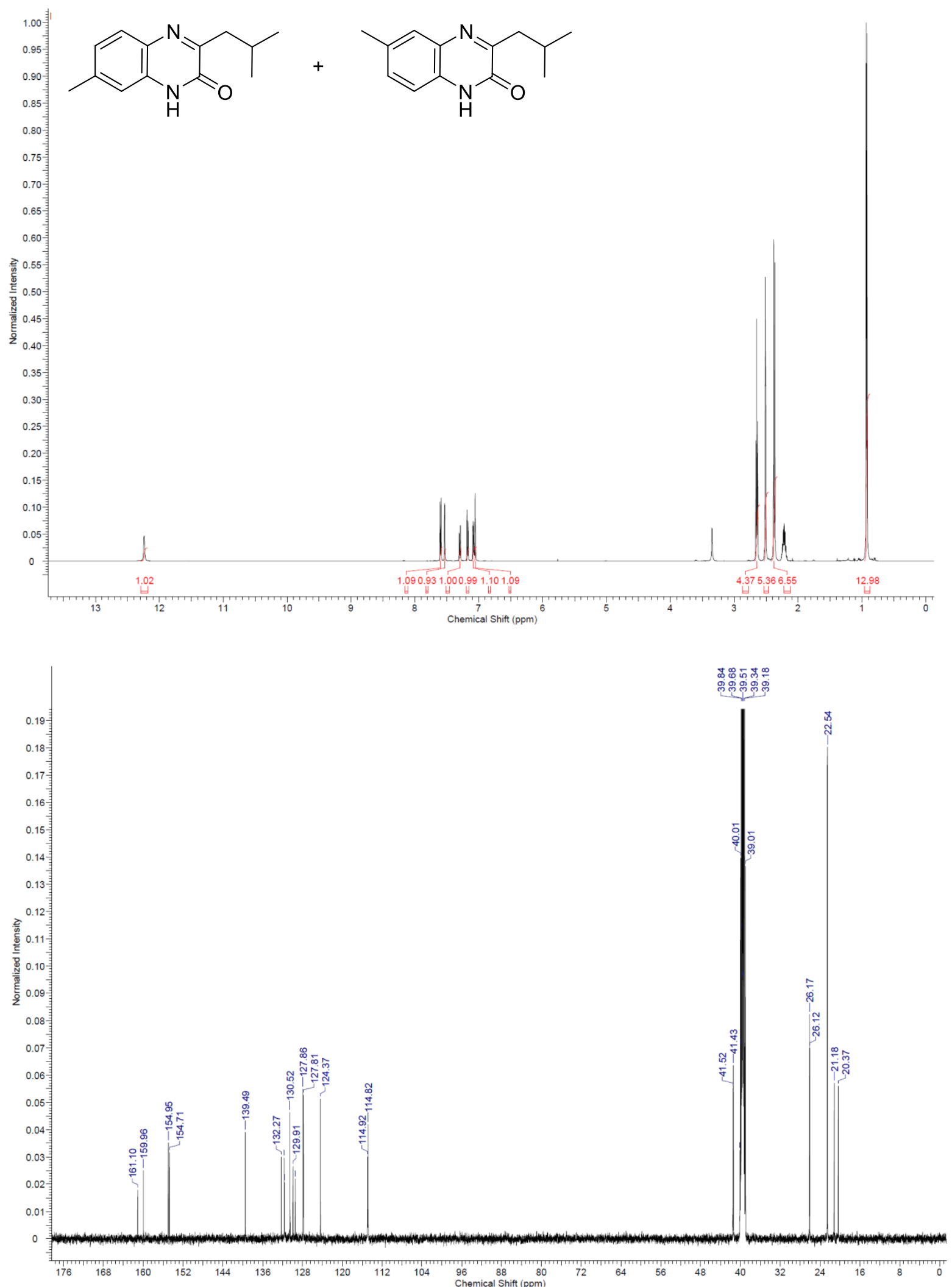


\section{Compound 5cc}
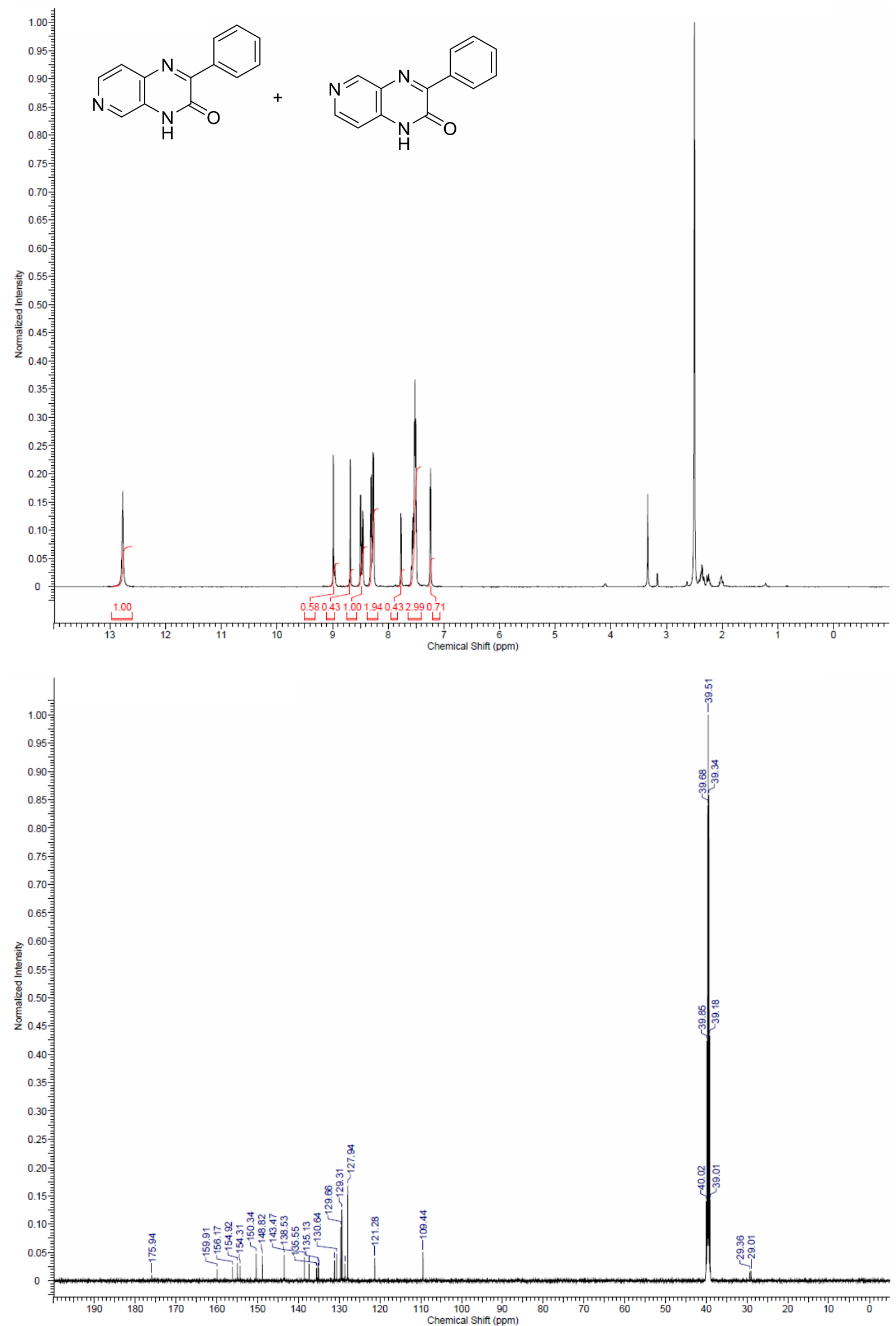
Compound 5ad
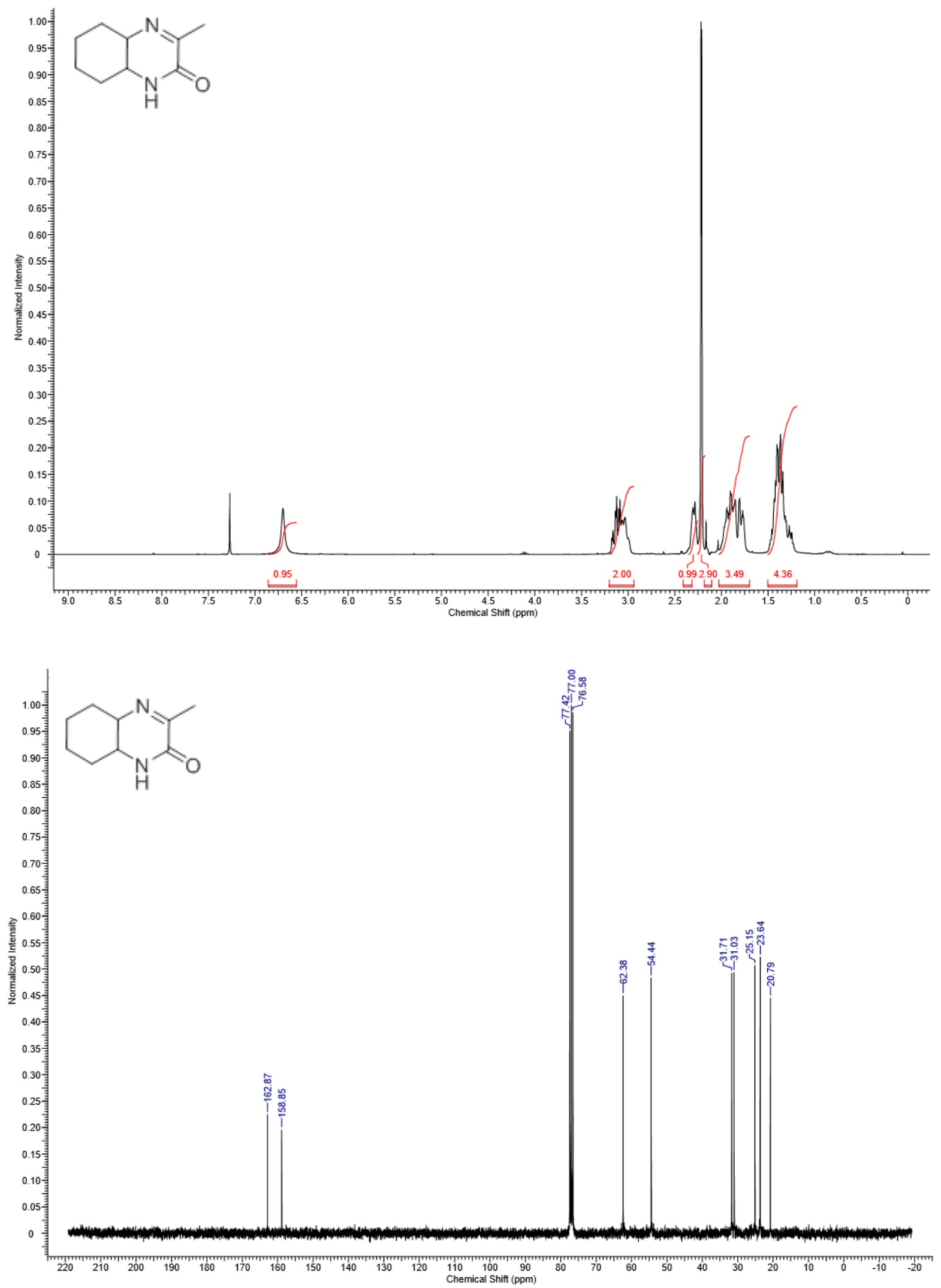
Compound 5bd
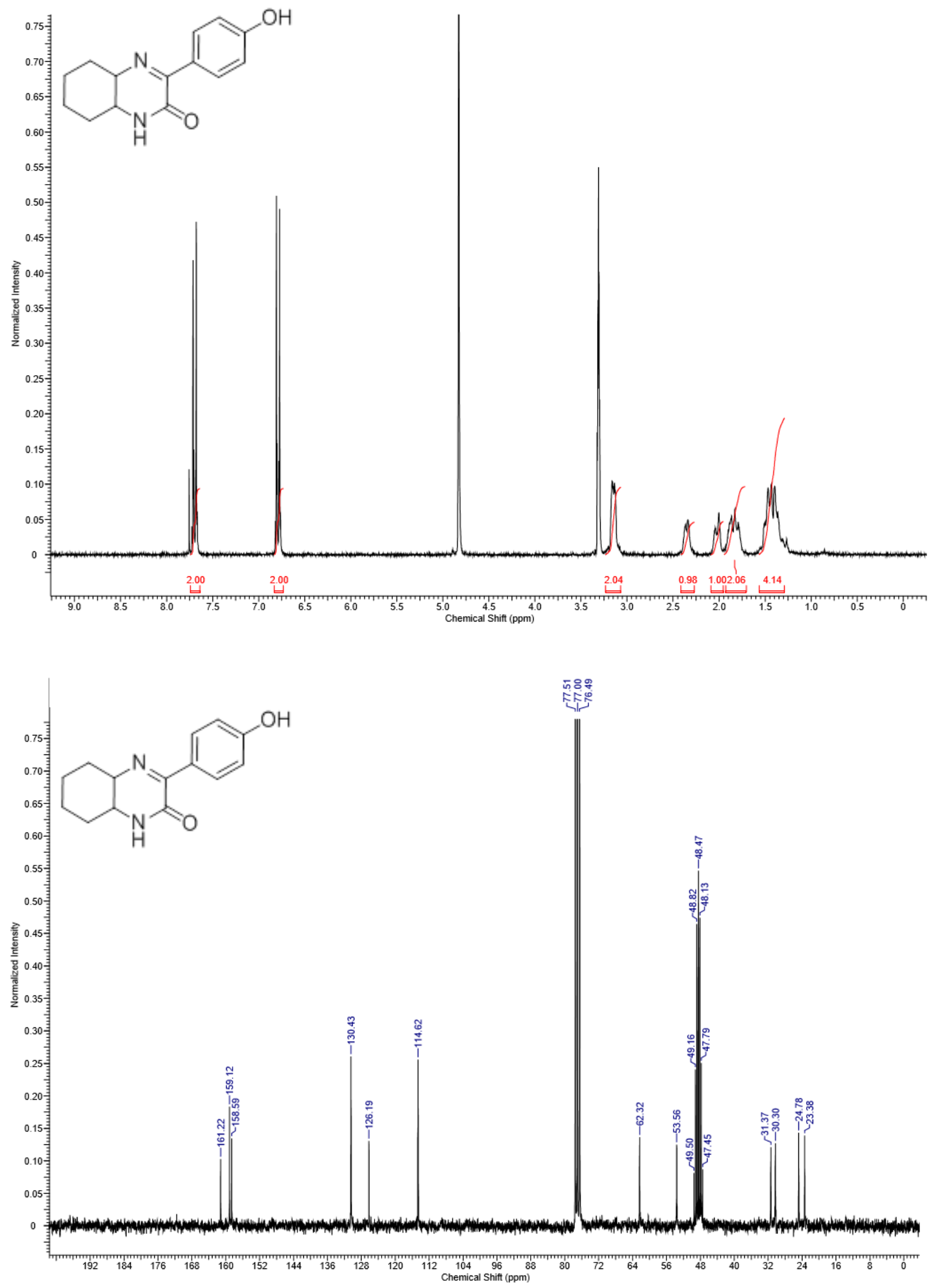


\section{Compound $5 \mathrm{~cd}$}
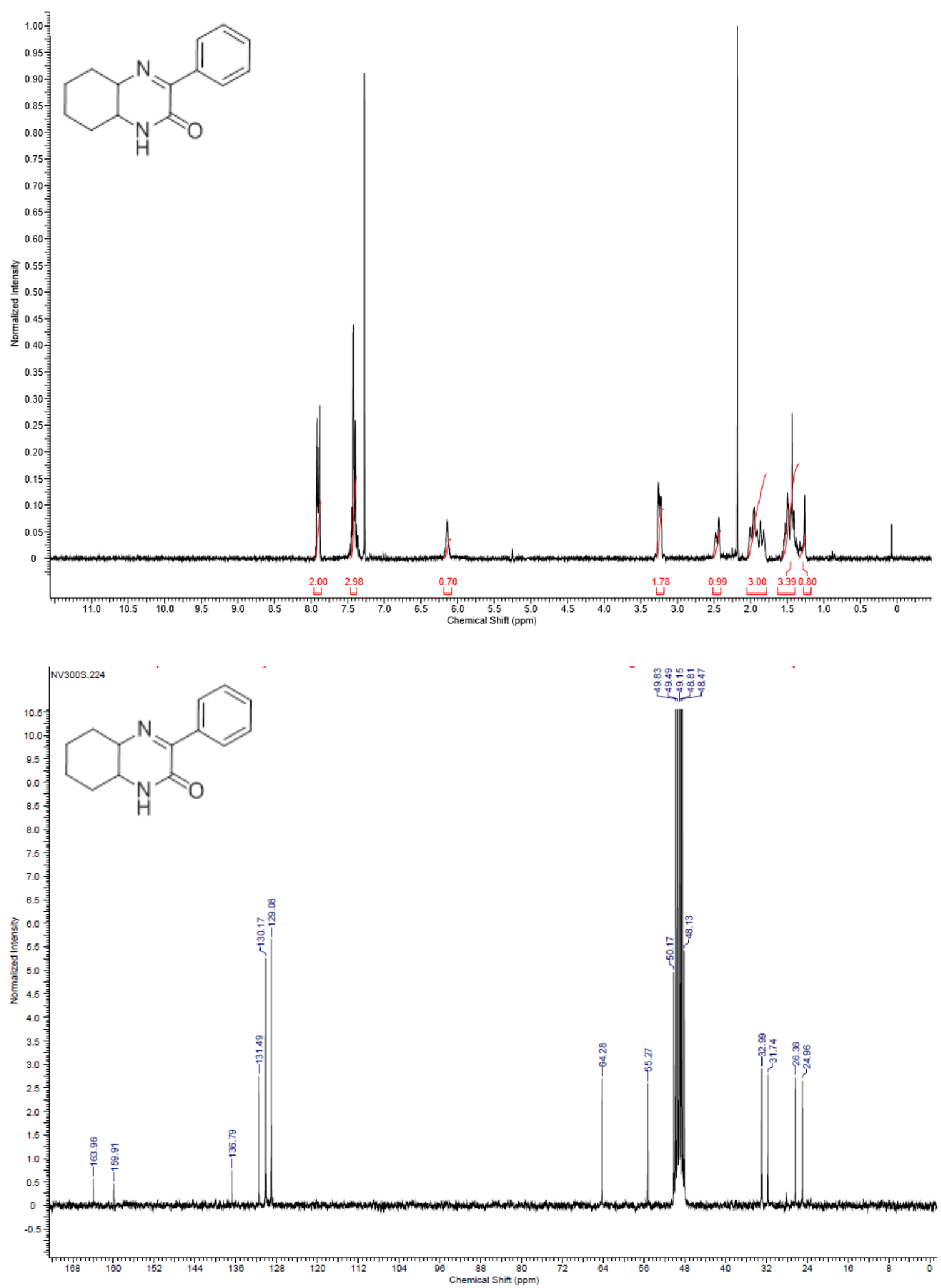


\section{Compound 5dd}
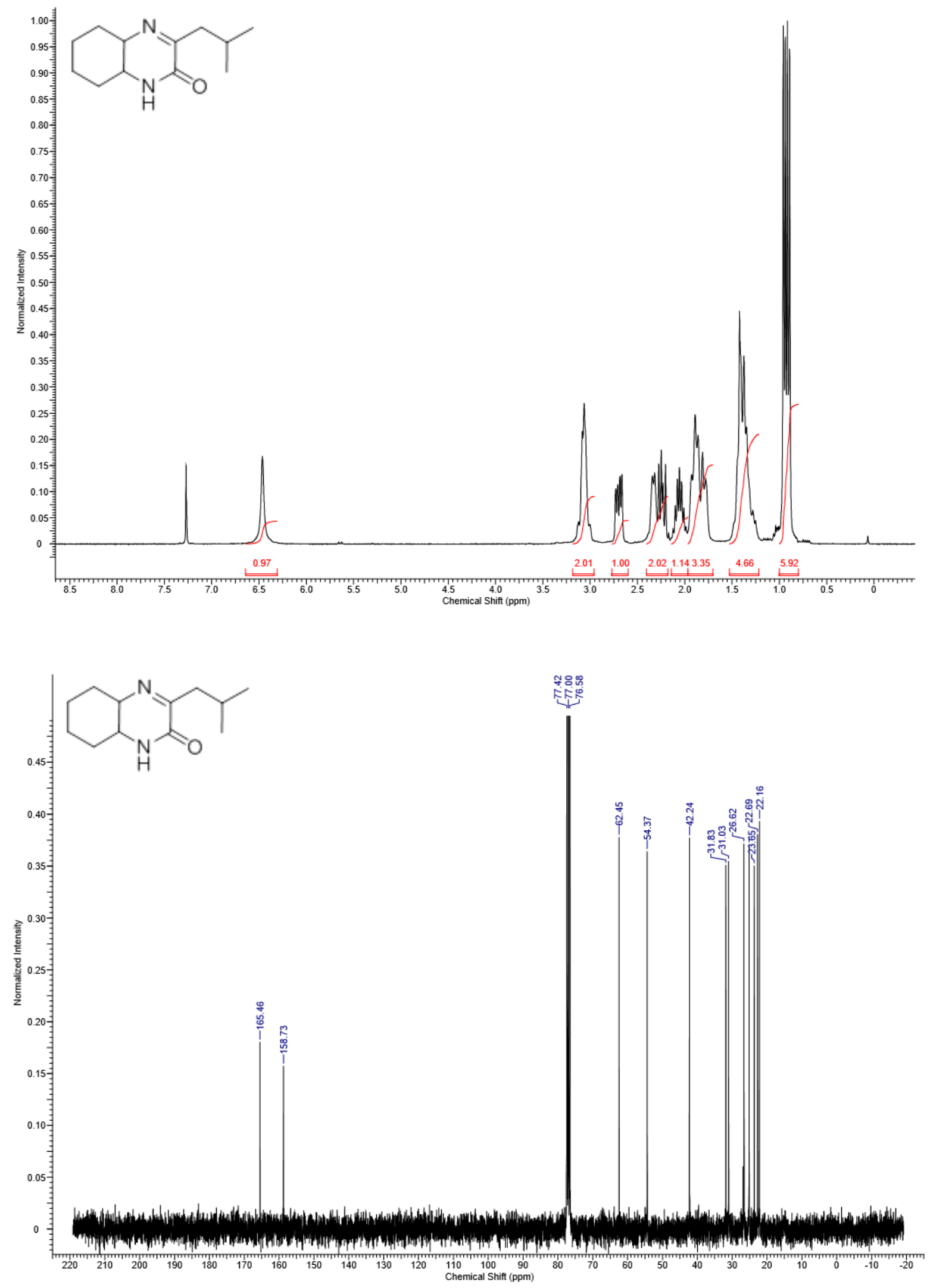


\section{Compound 5ed}
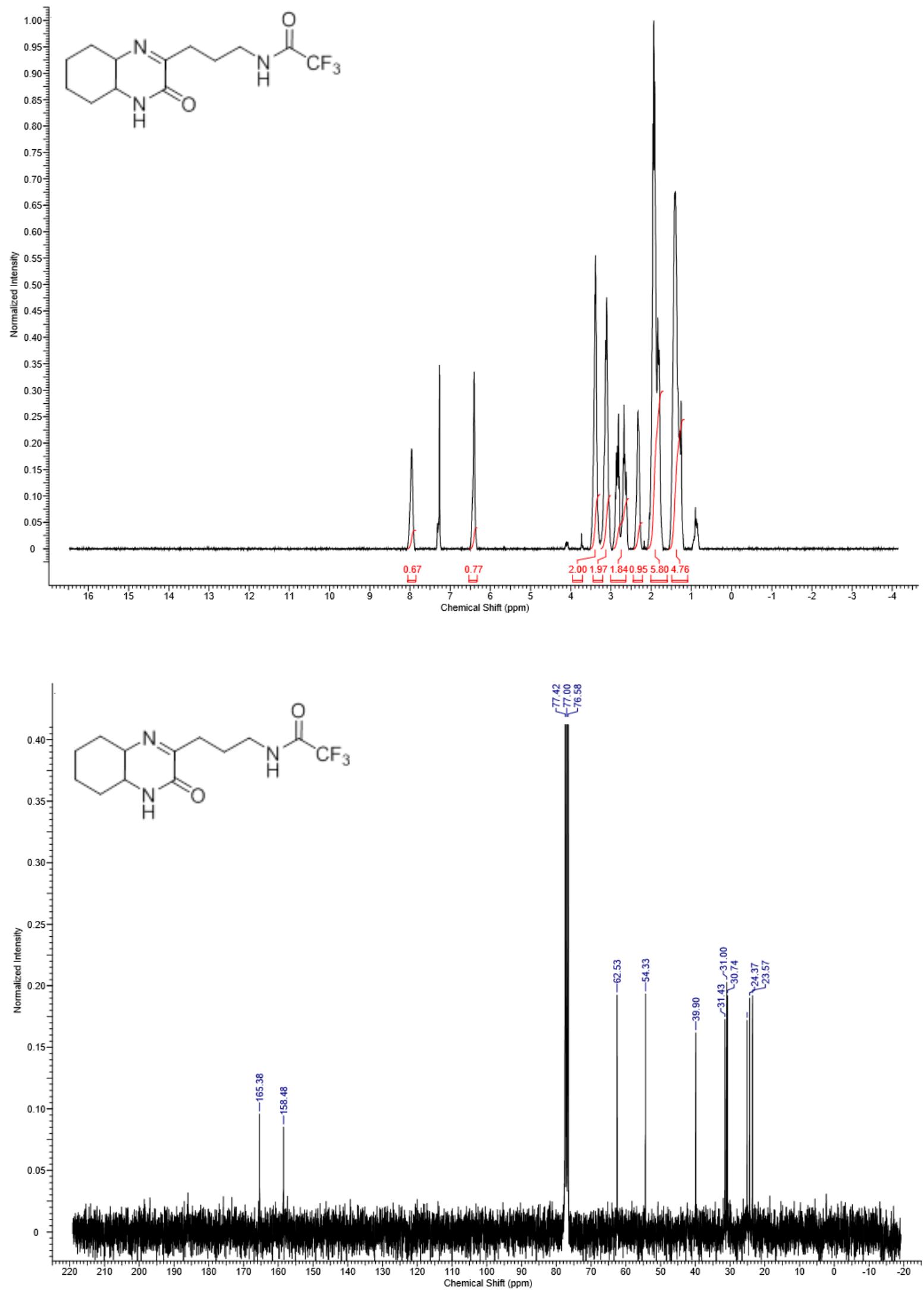


\section{Compound 5de}
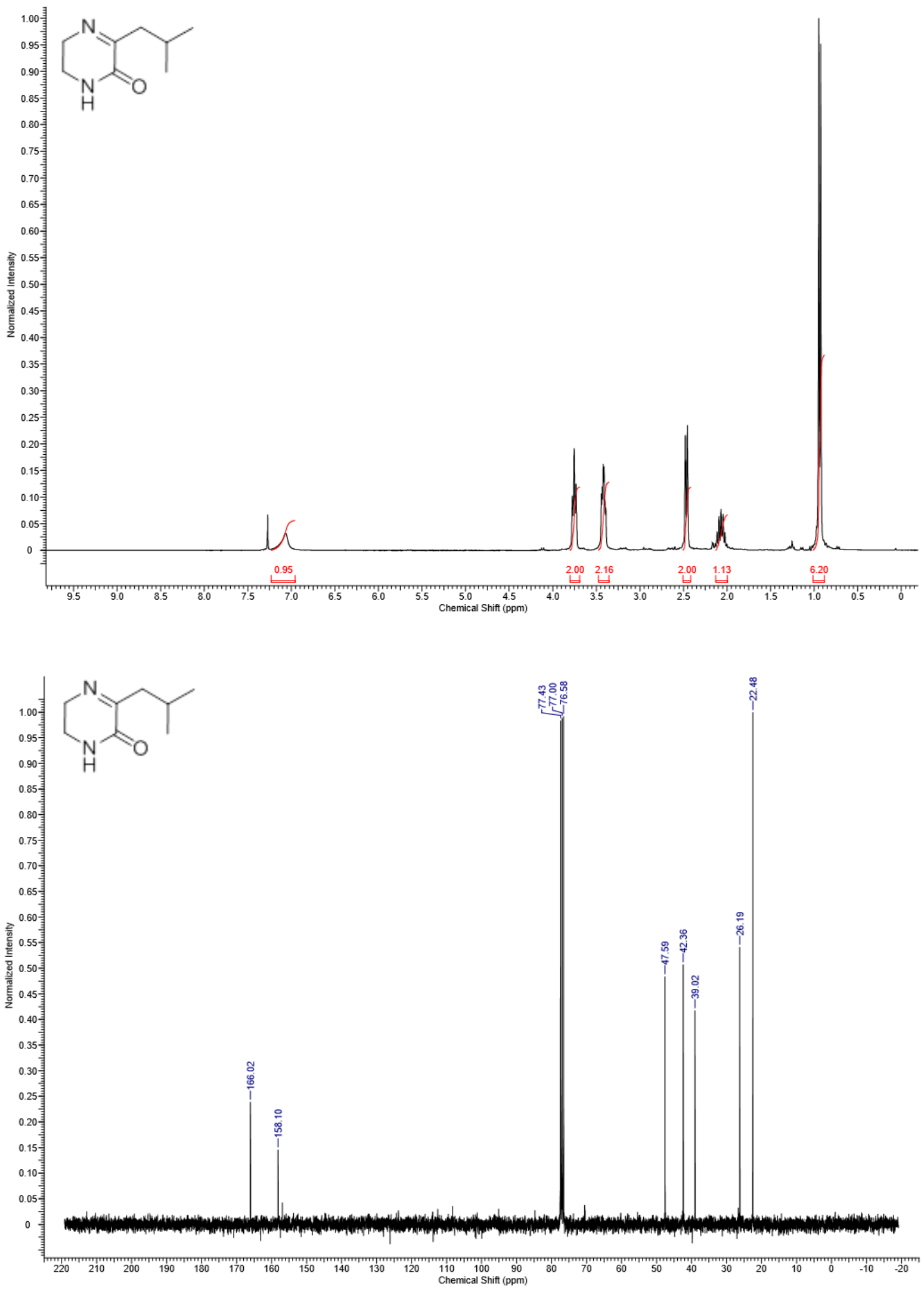


\section{Compound 5be}
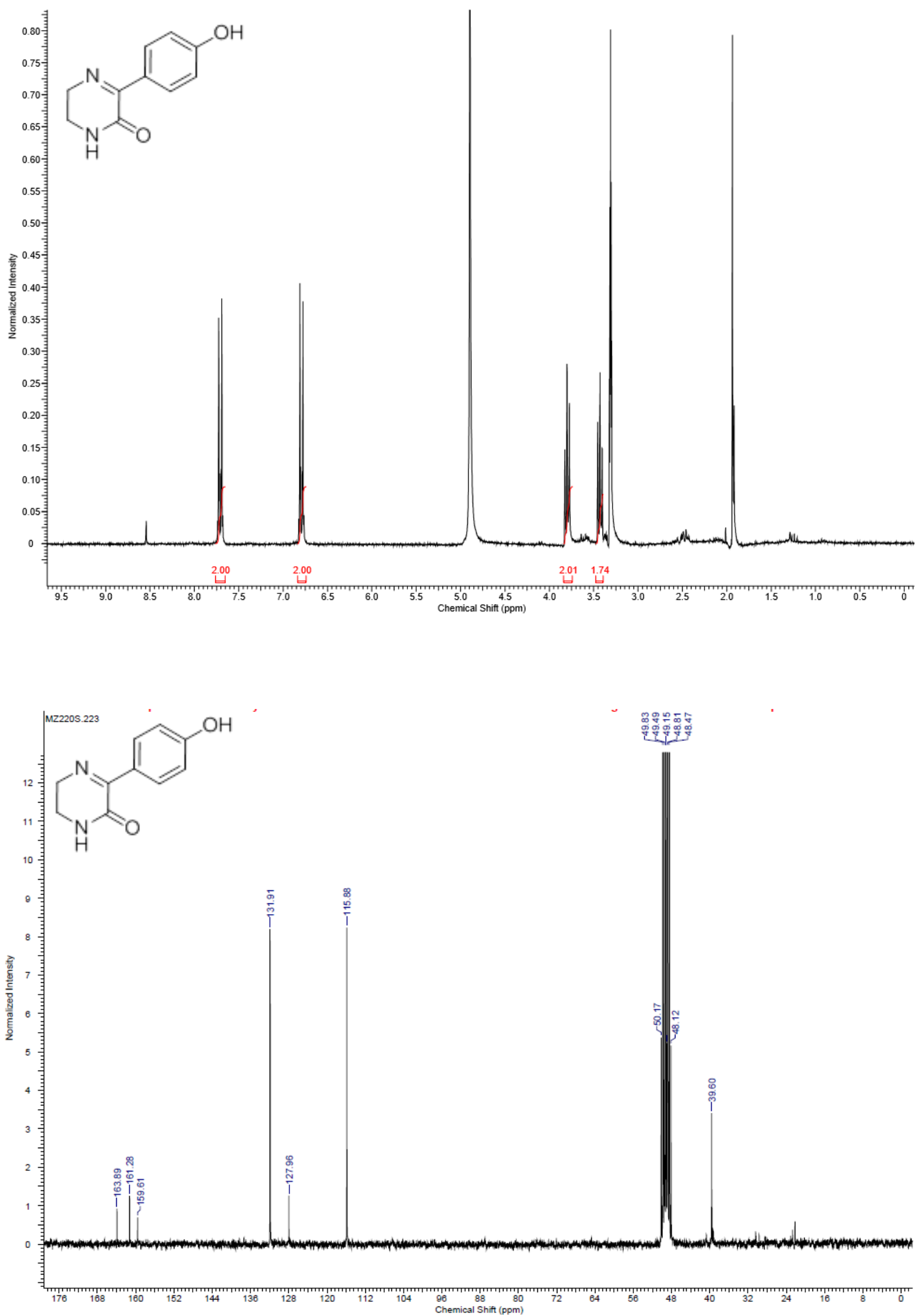


\section{Compound 5bf}
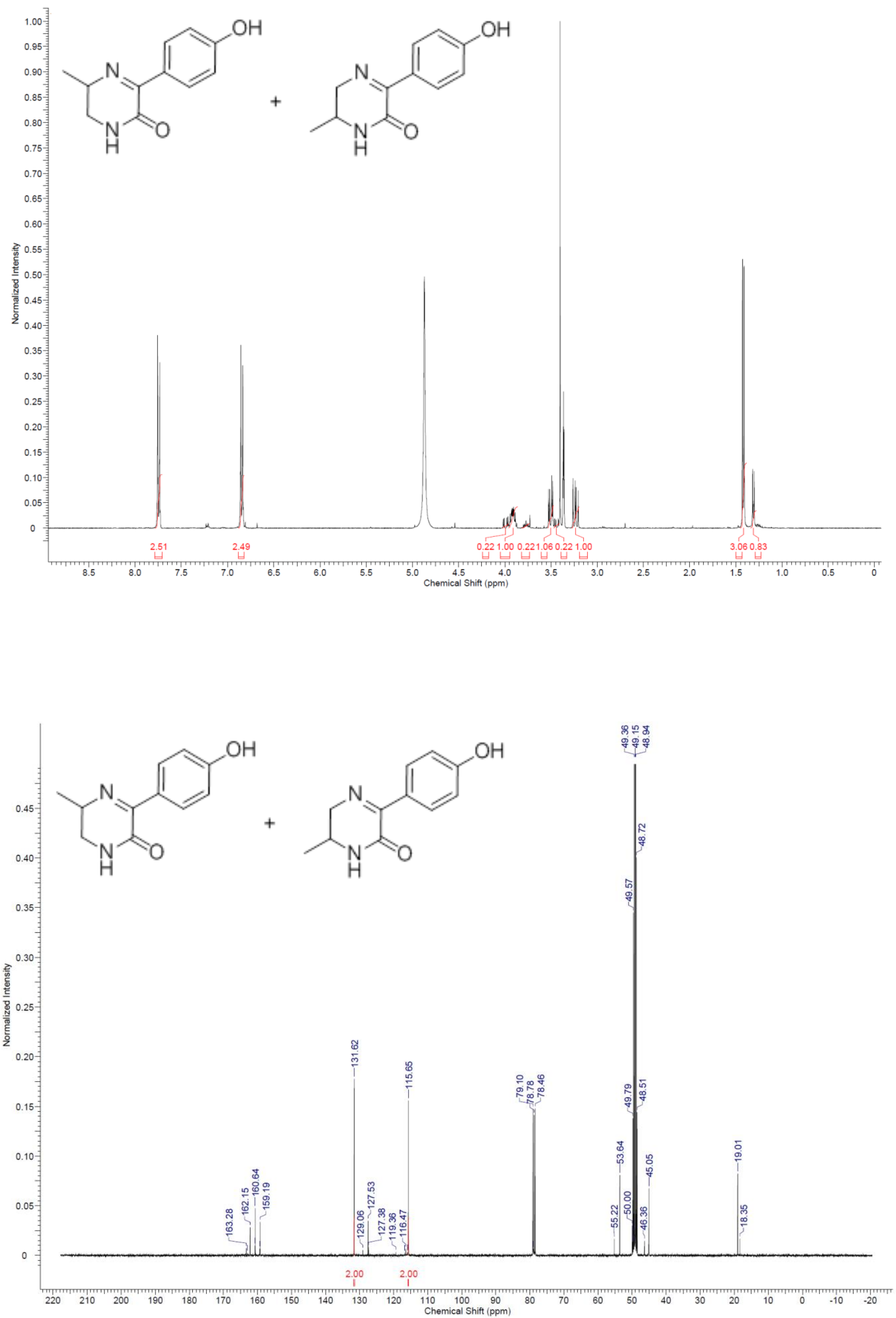


\section{Compound 5ce}
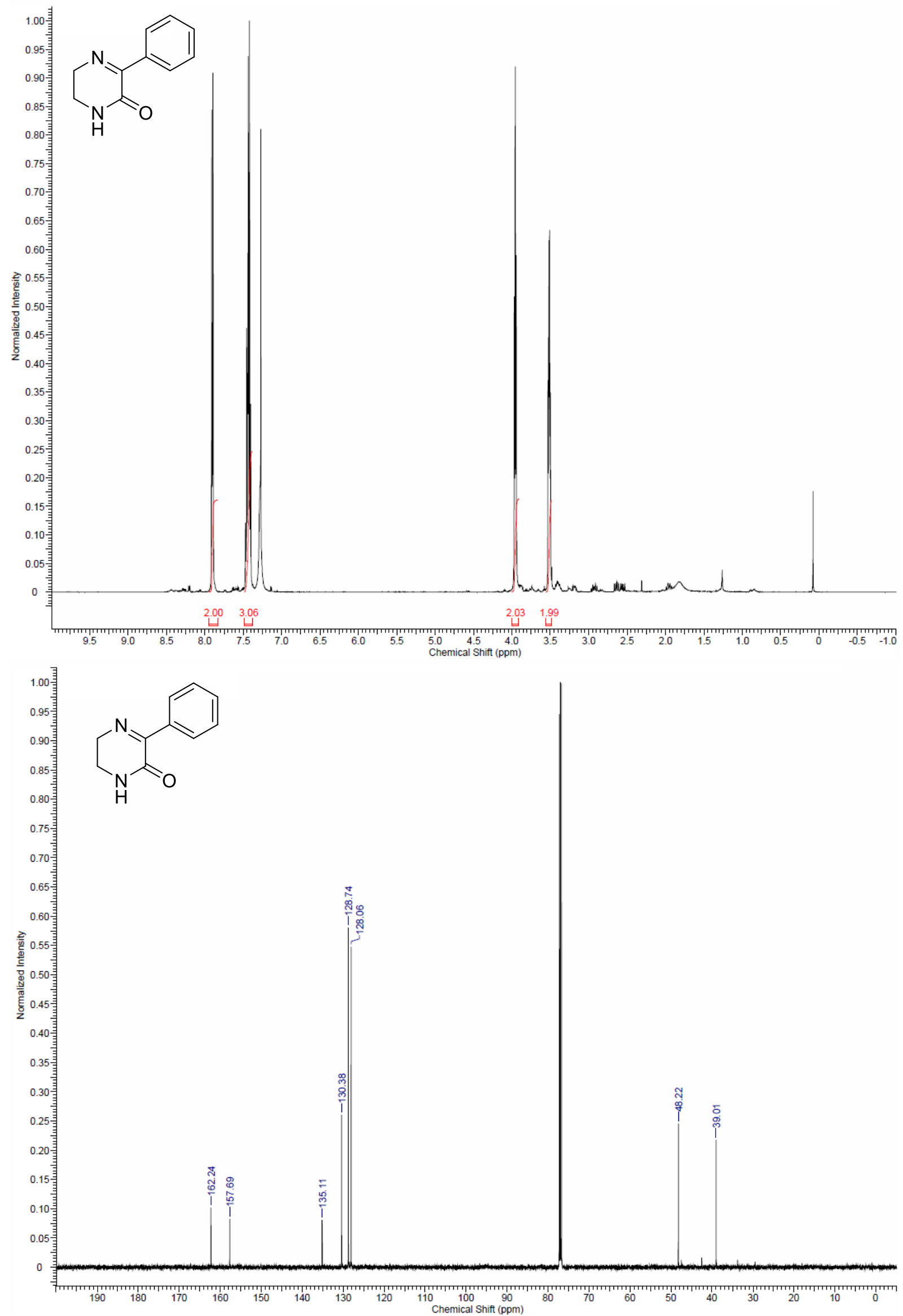


\section{Compound 5cf}
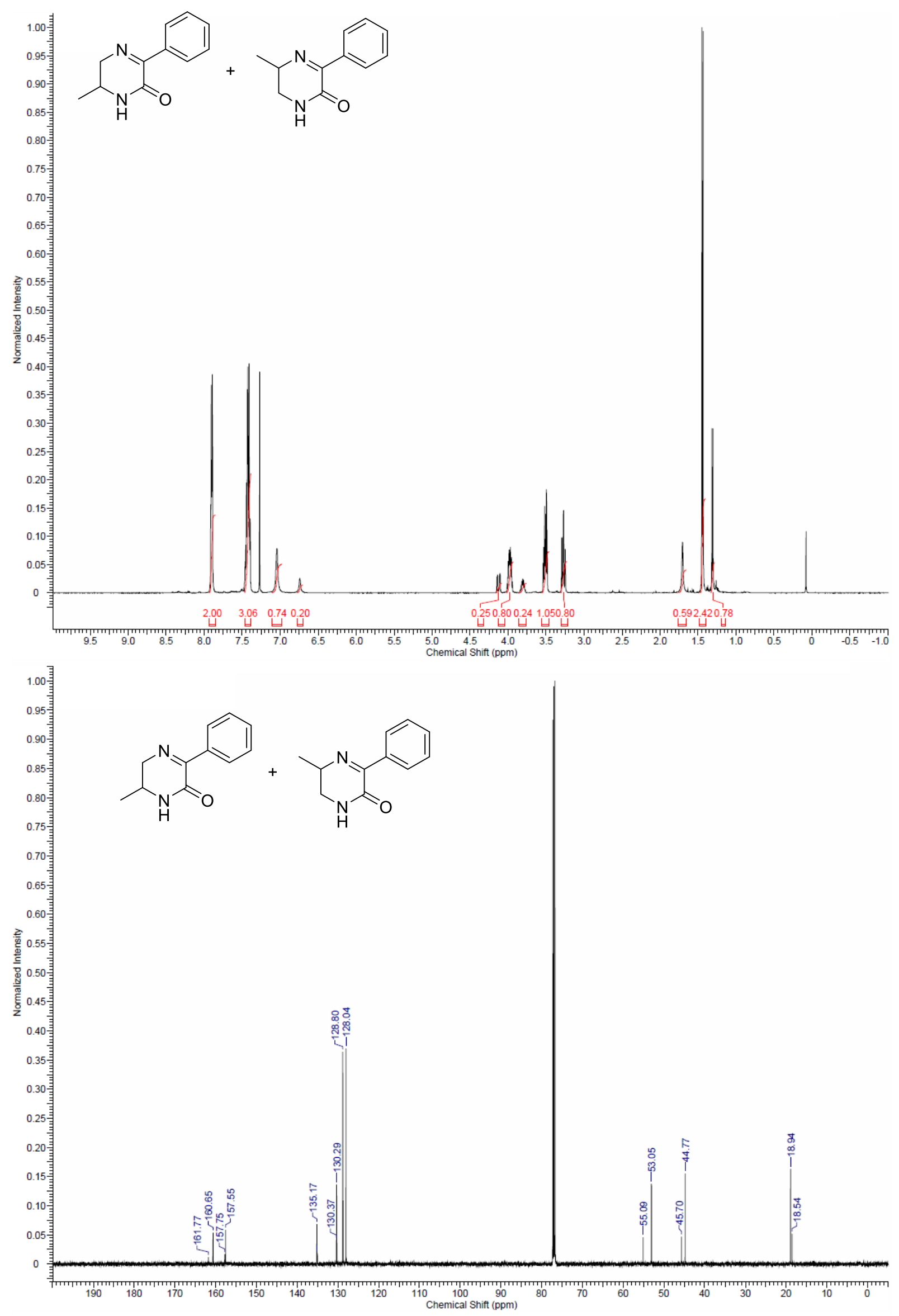


\section{Compound 7aa}
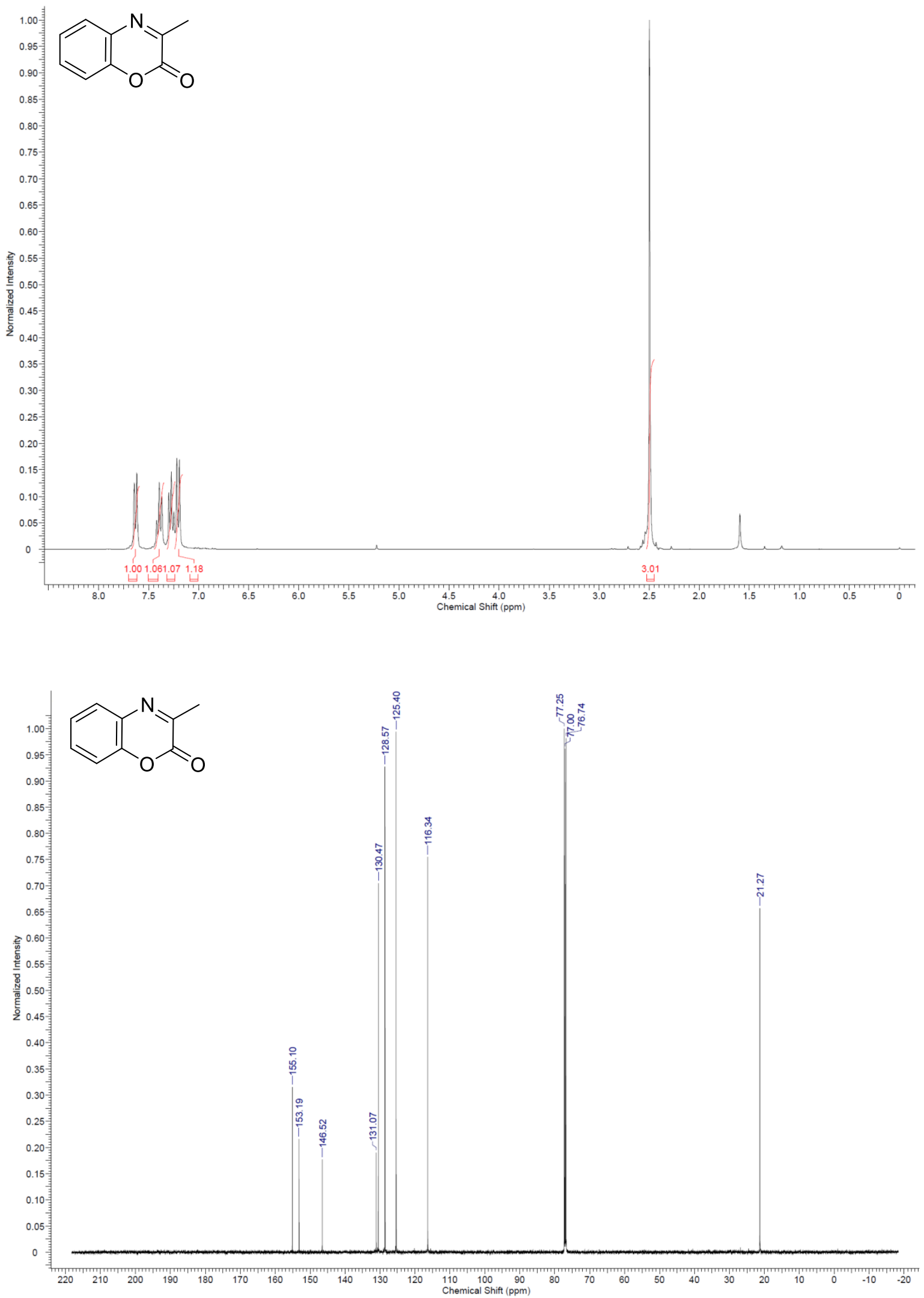


\section{Compound 7ab}
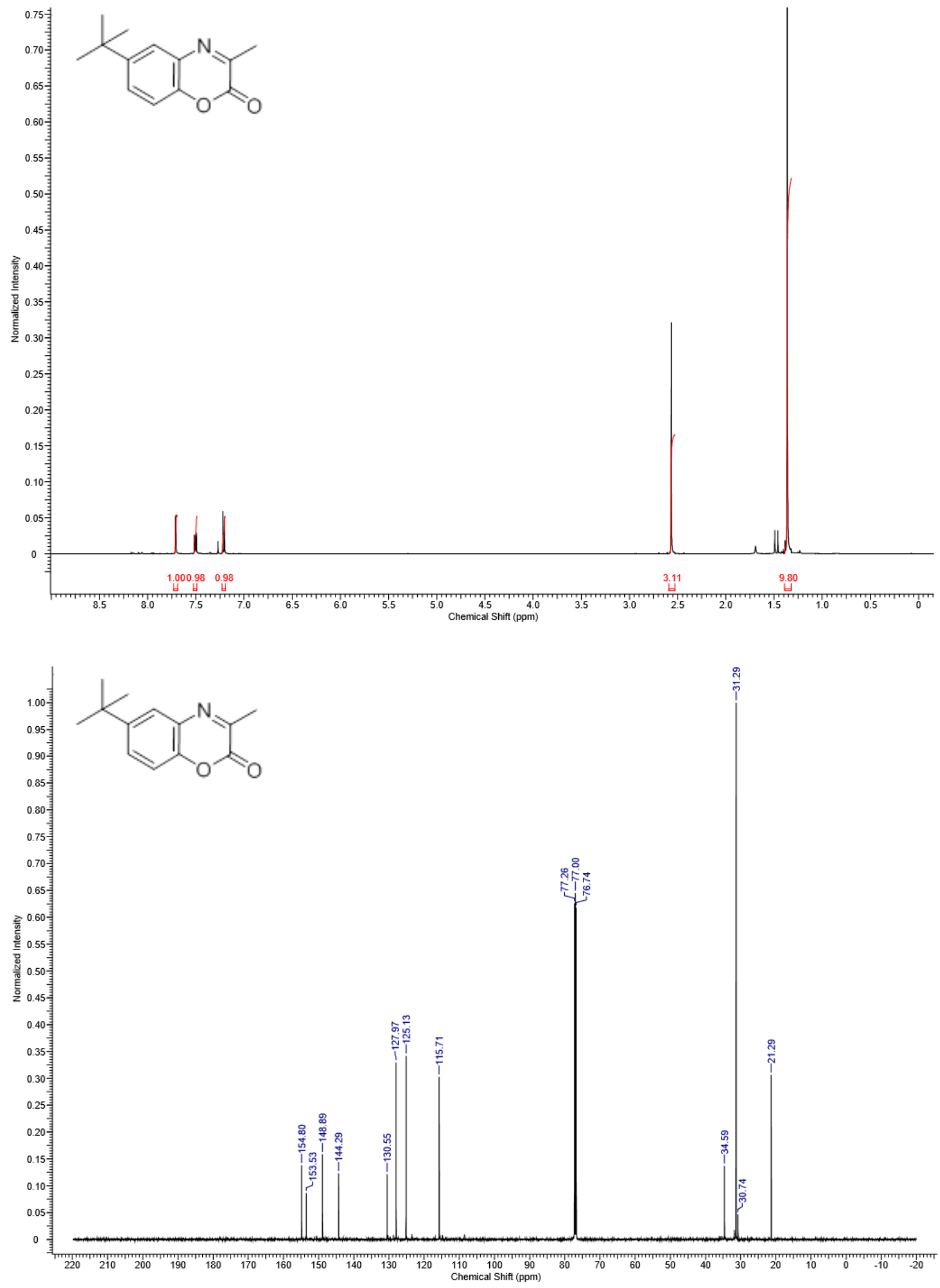


\section{Compound $7 \mathrm{ba}$}
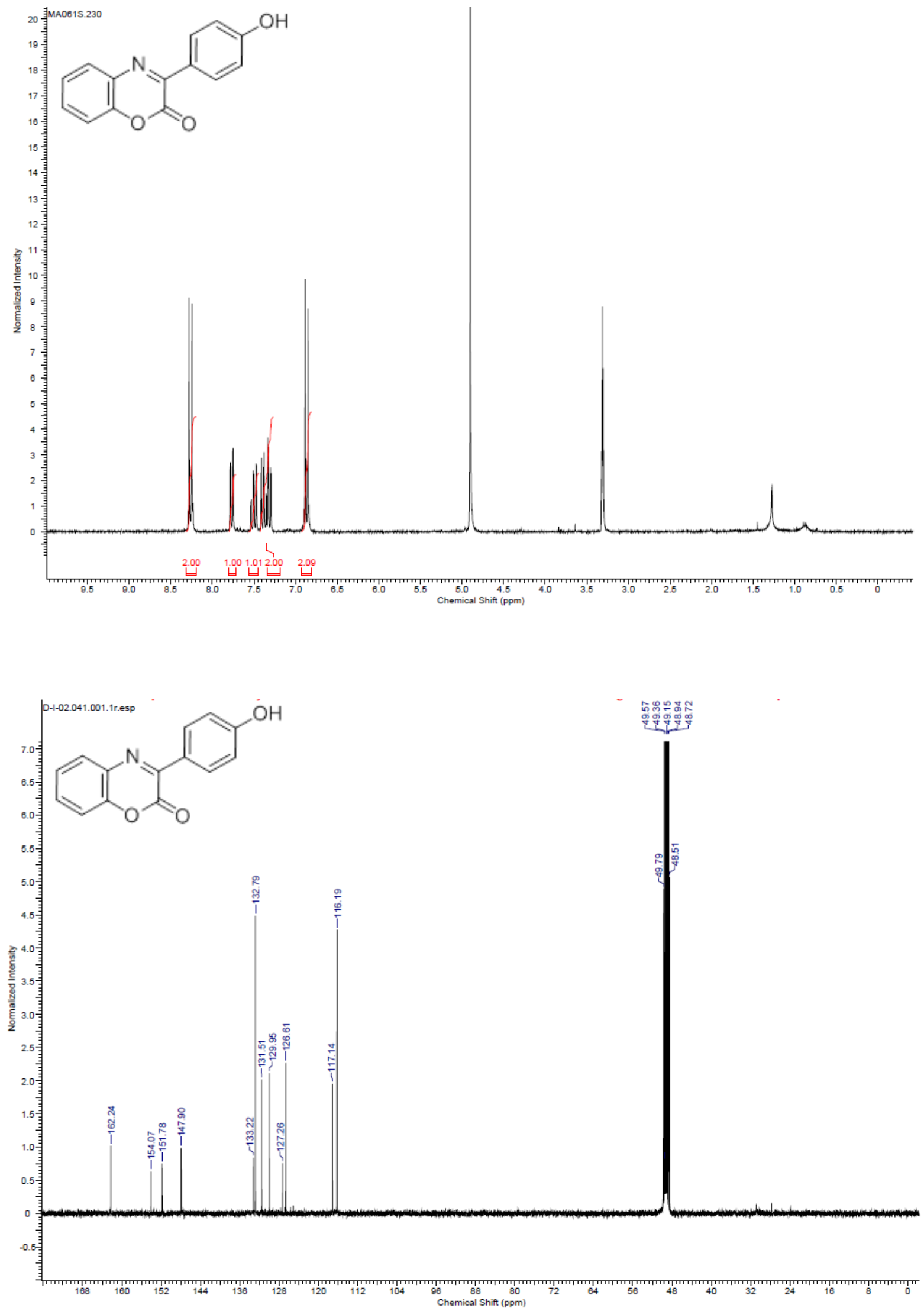


\section{Compound 7bb}
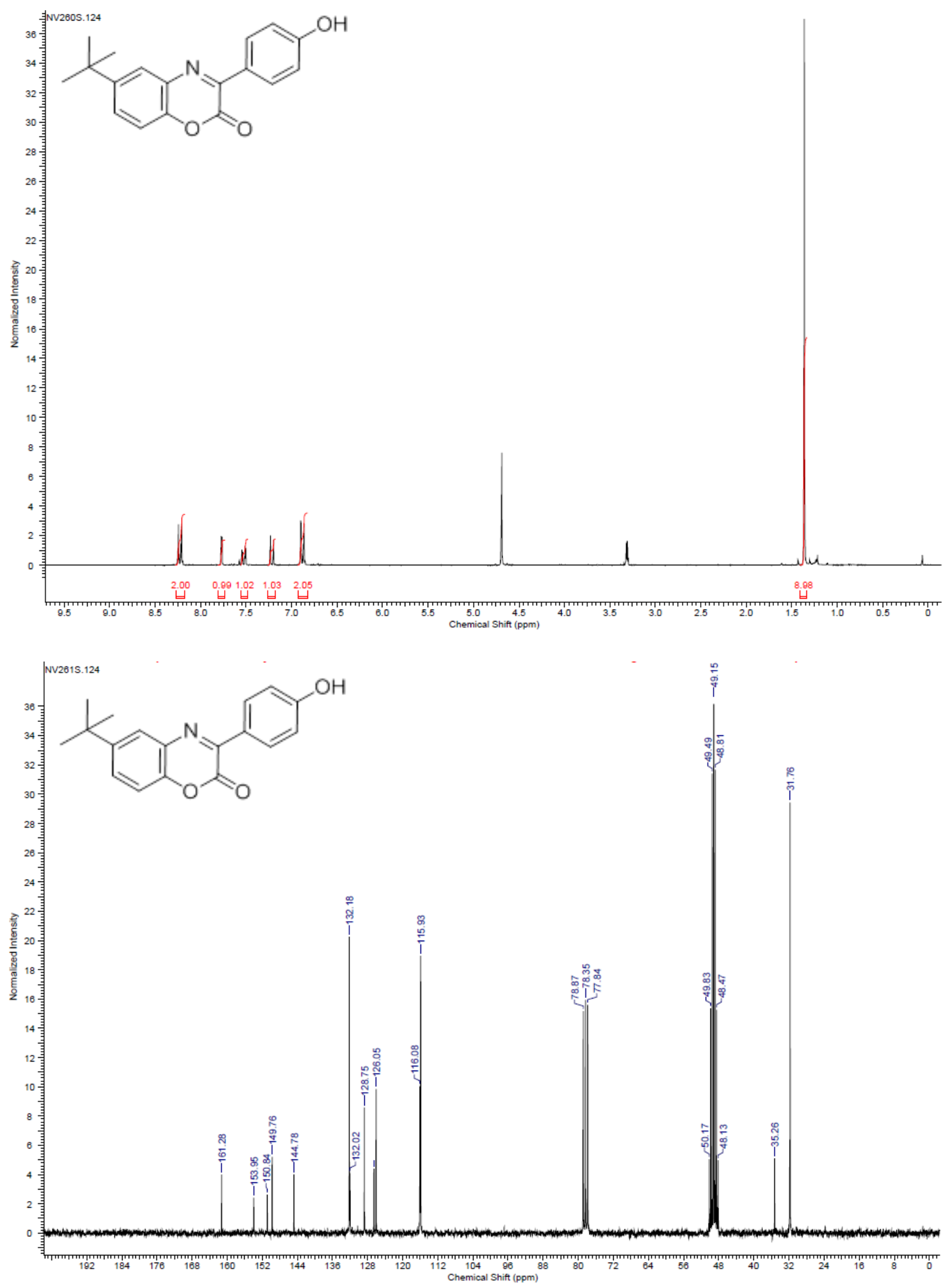


\section{Compound 7bc}
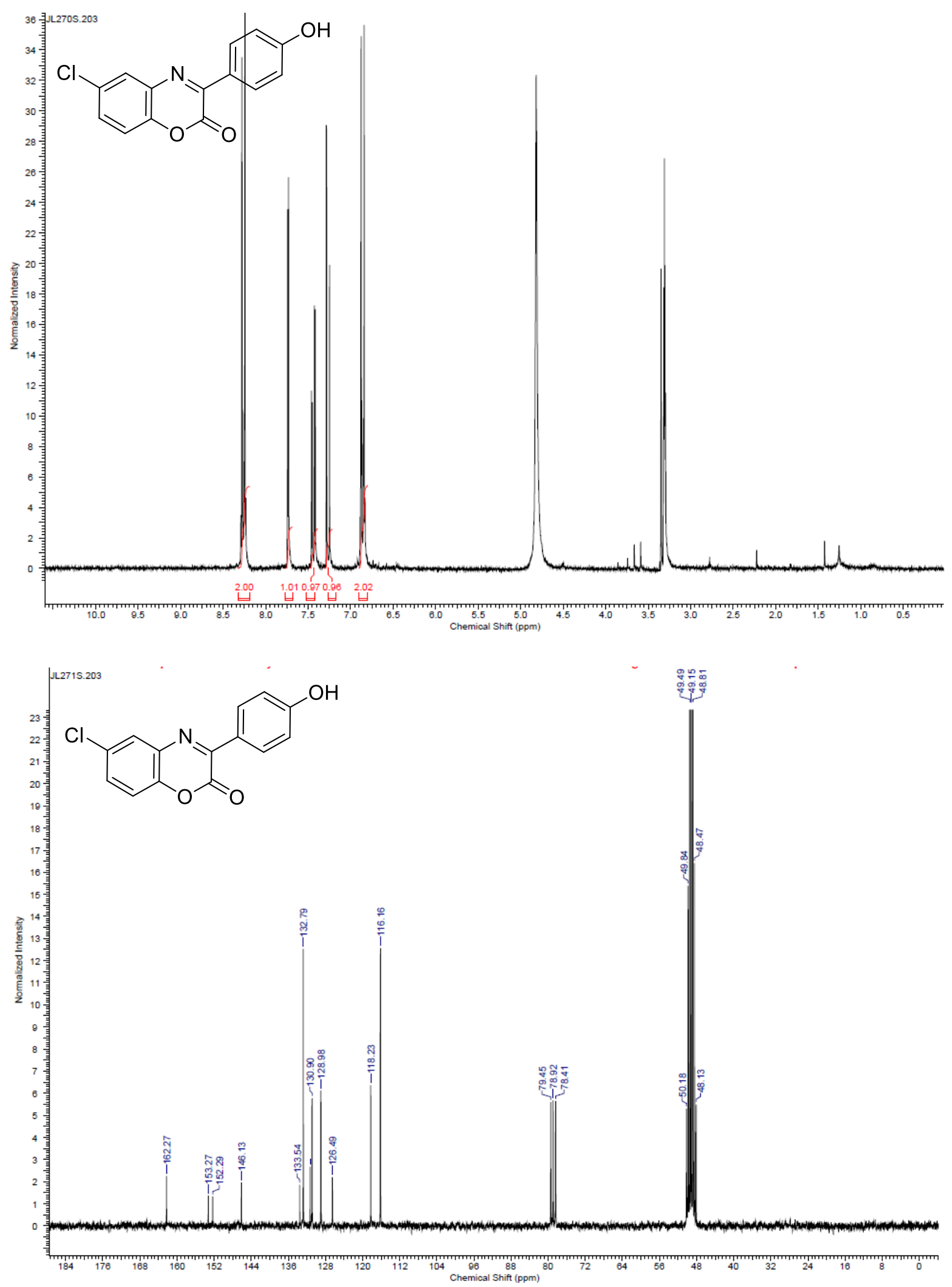

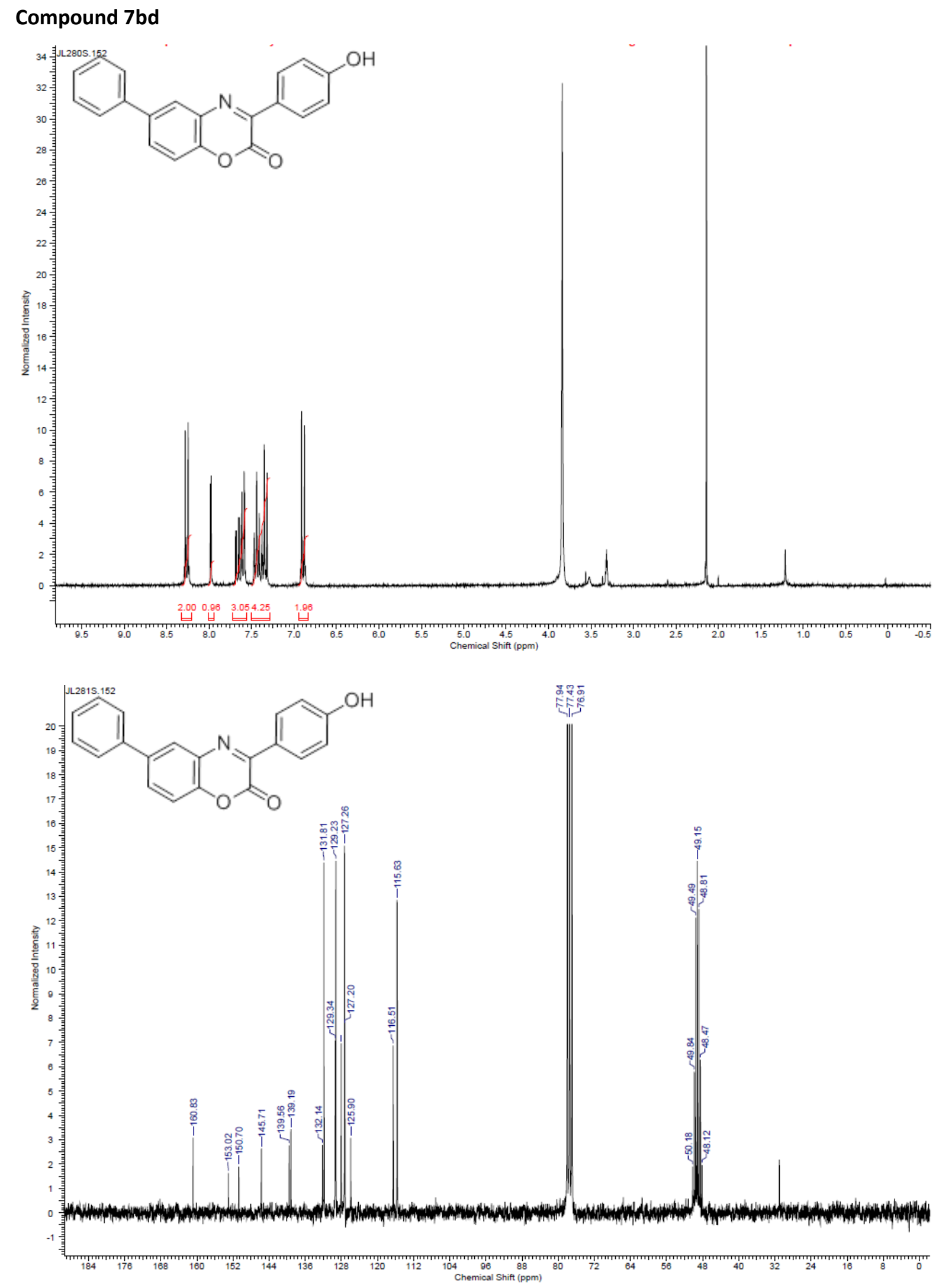


\section{Compound 7be}
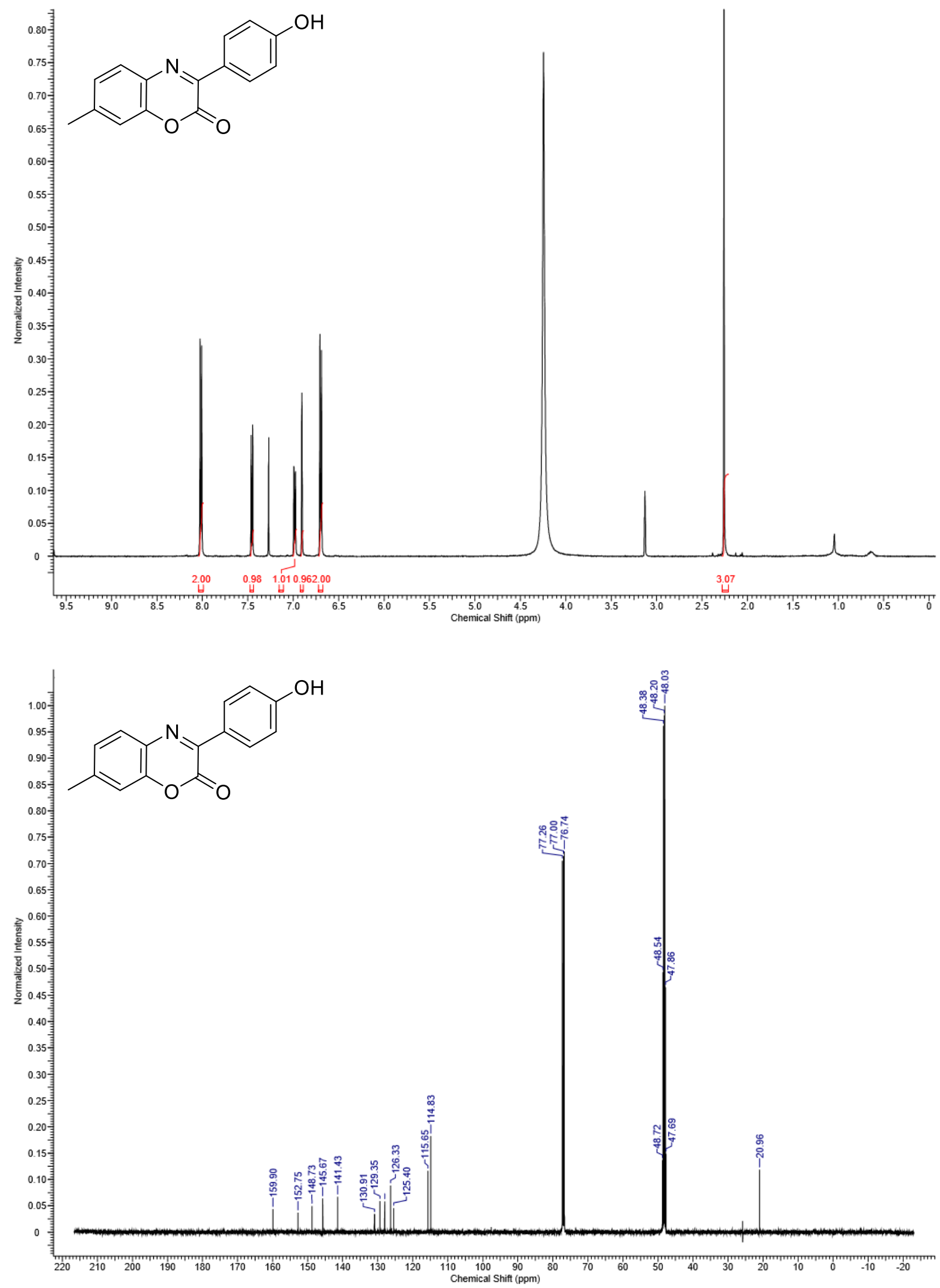
Compound 7cb
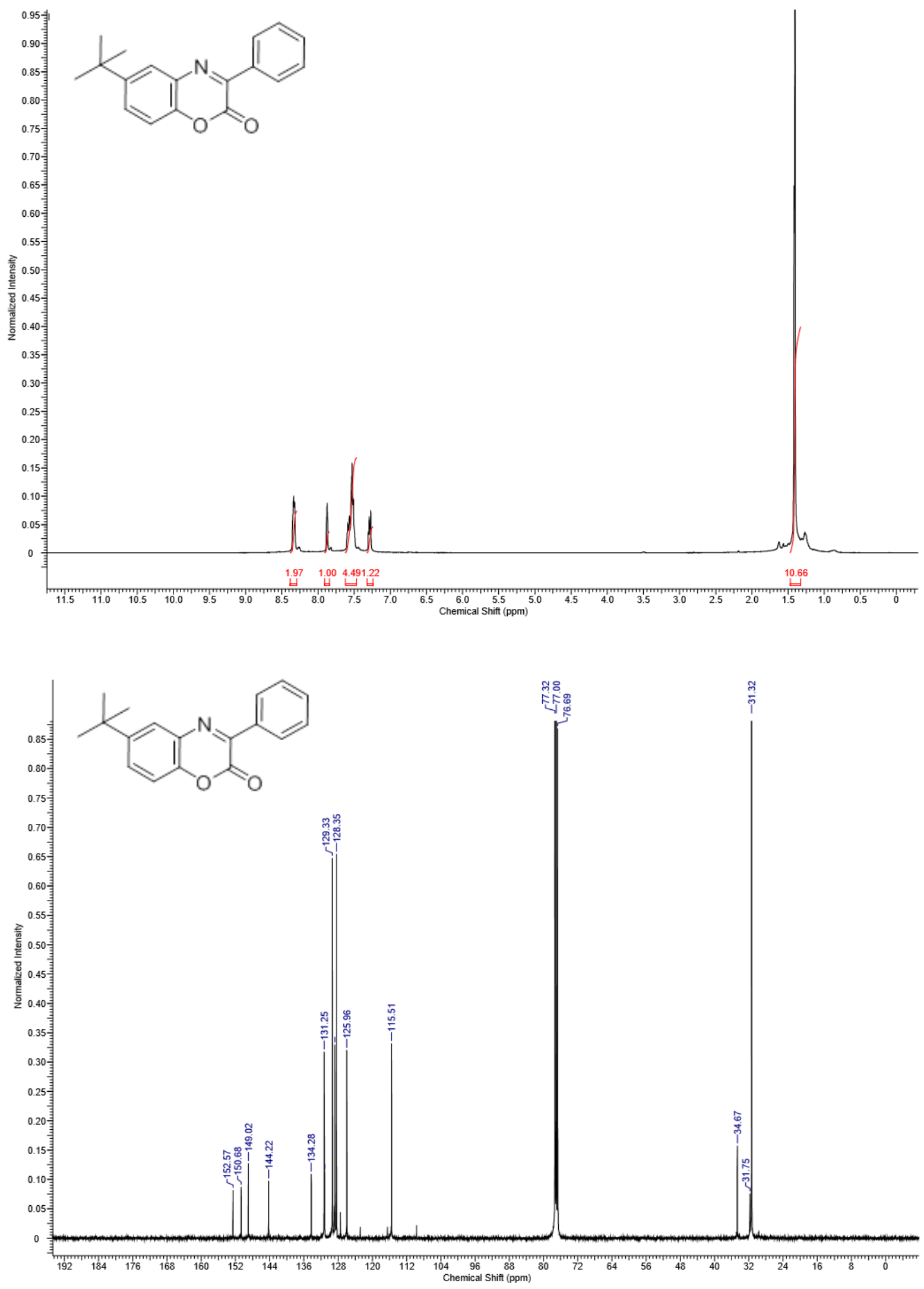


\section{Compound 7da}
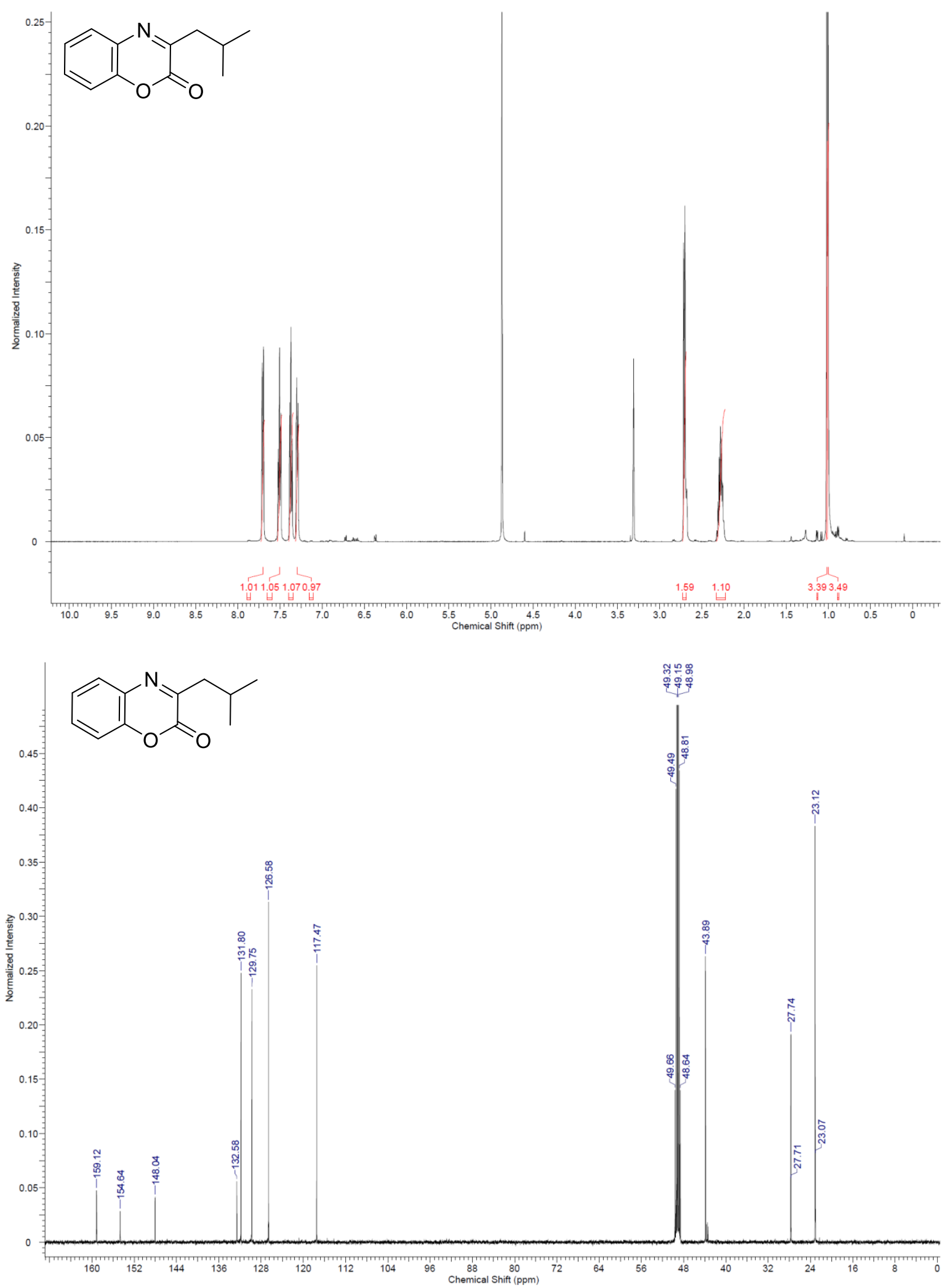


\section{Compound 7db}
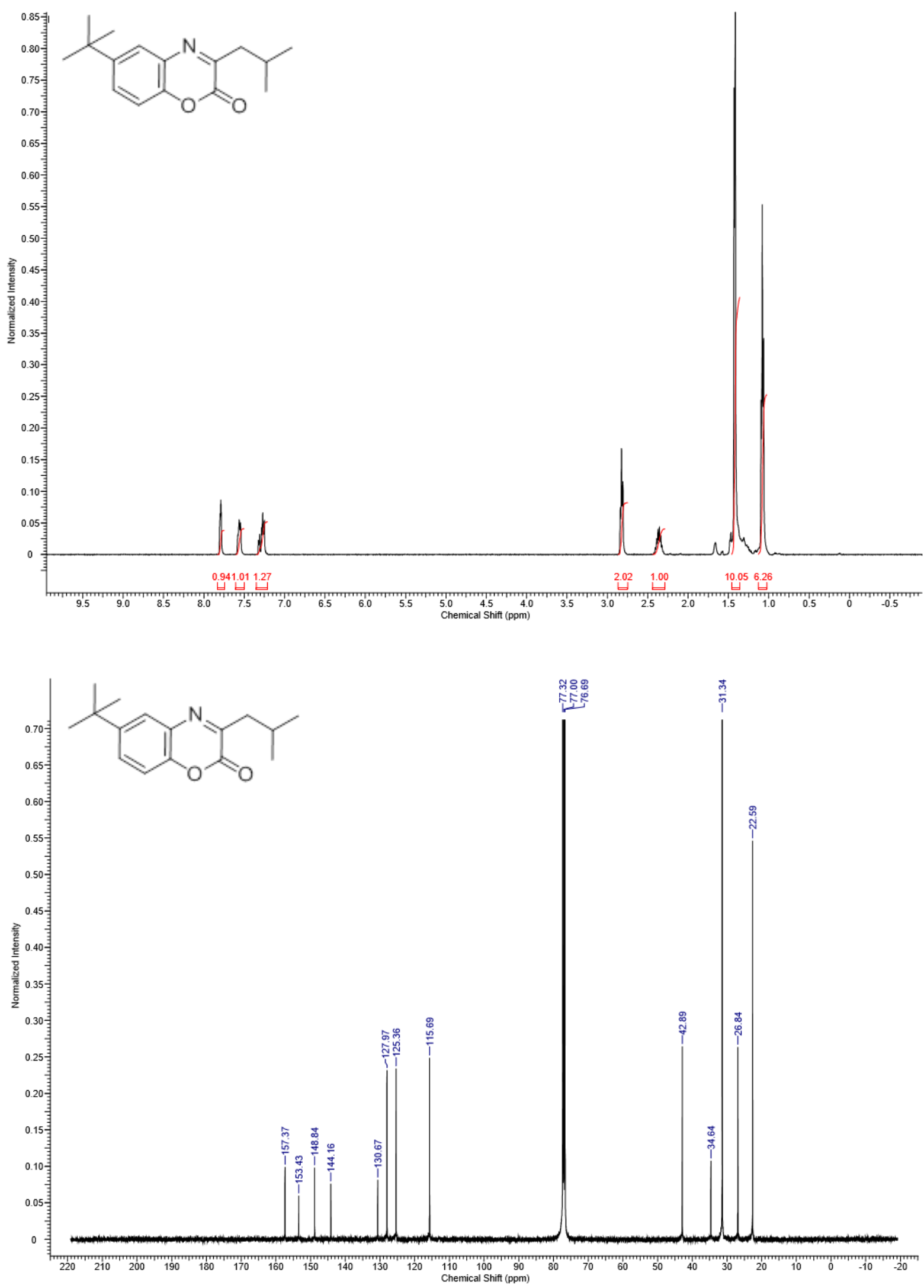


\section{Compound 7ea}
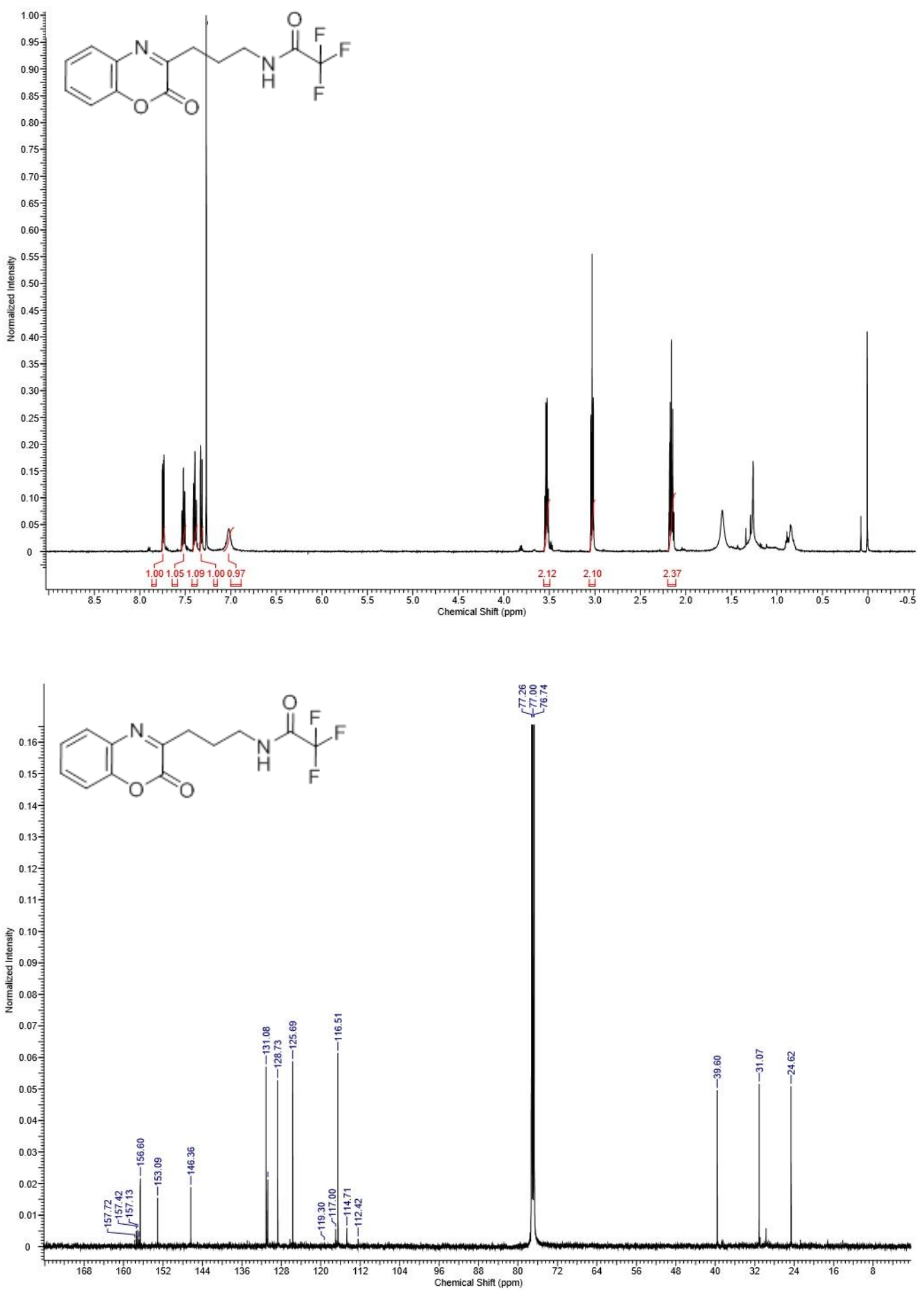


\section{Compound 7eb}
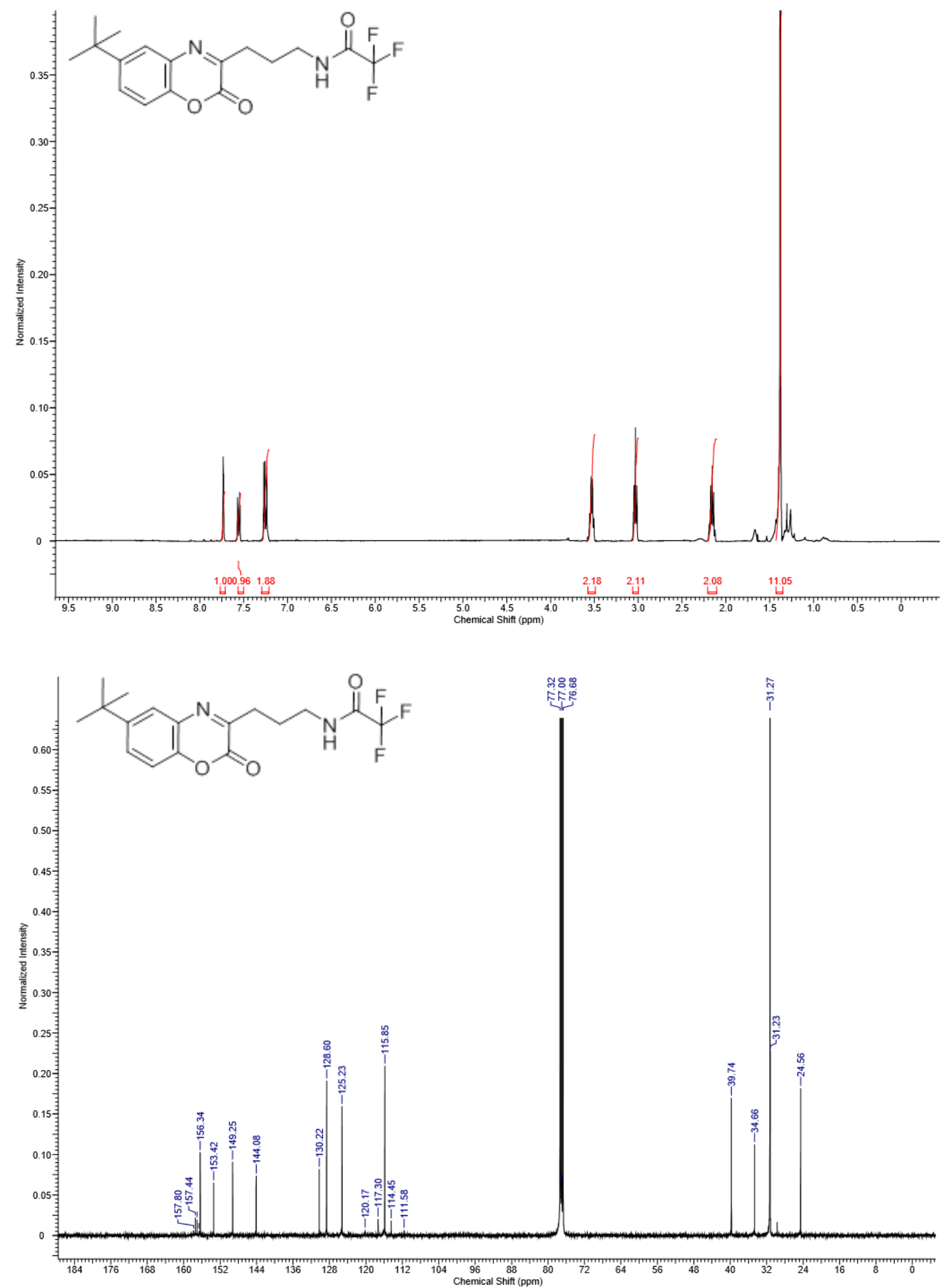


\section{Compound 7ec}
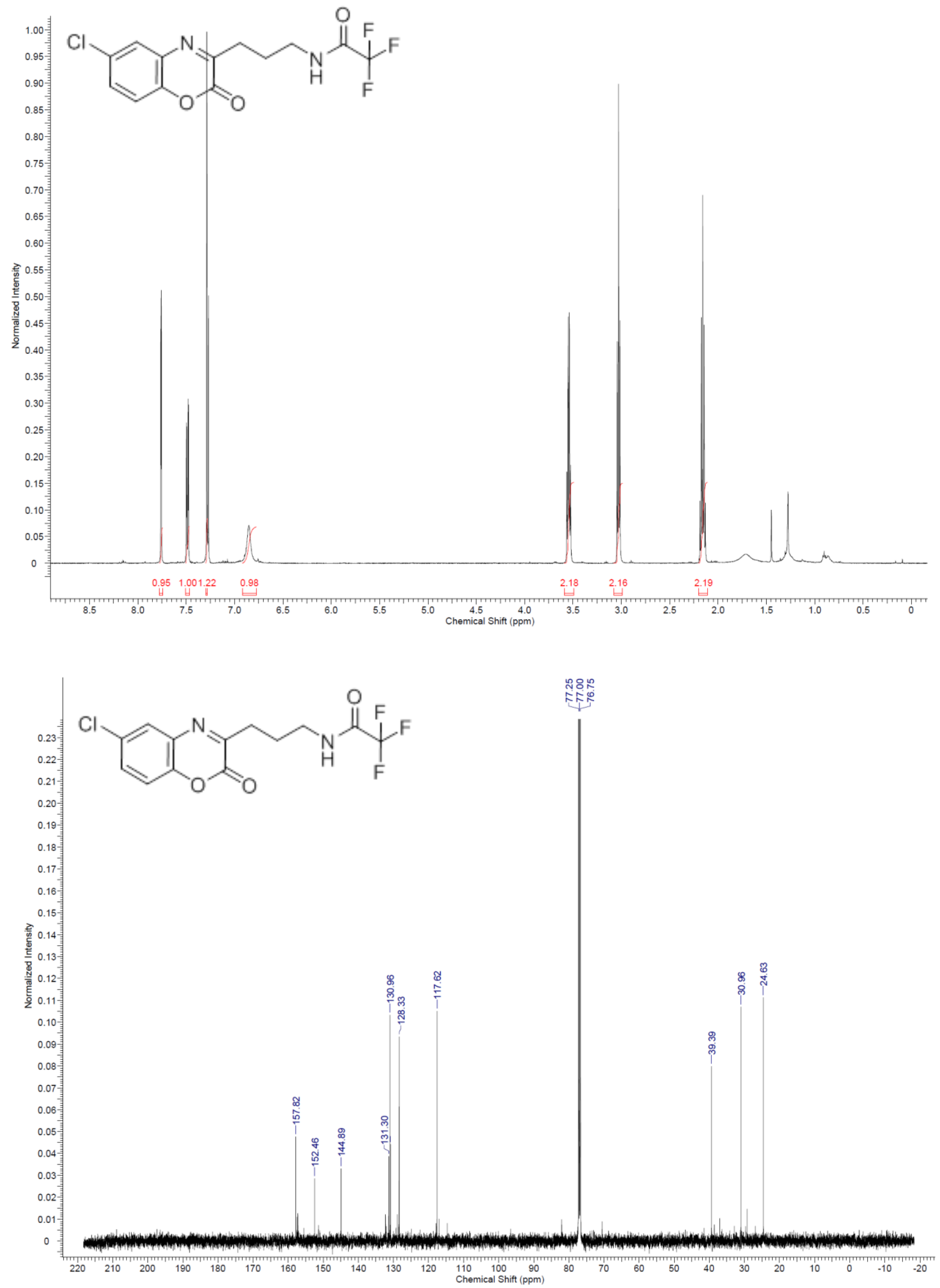


\section{Compound 7bf}
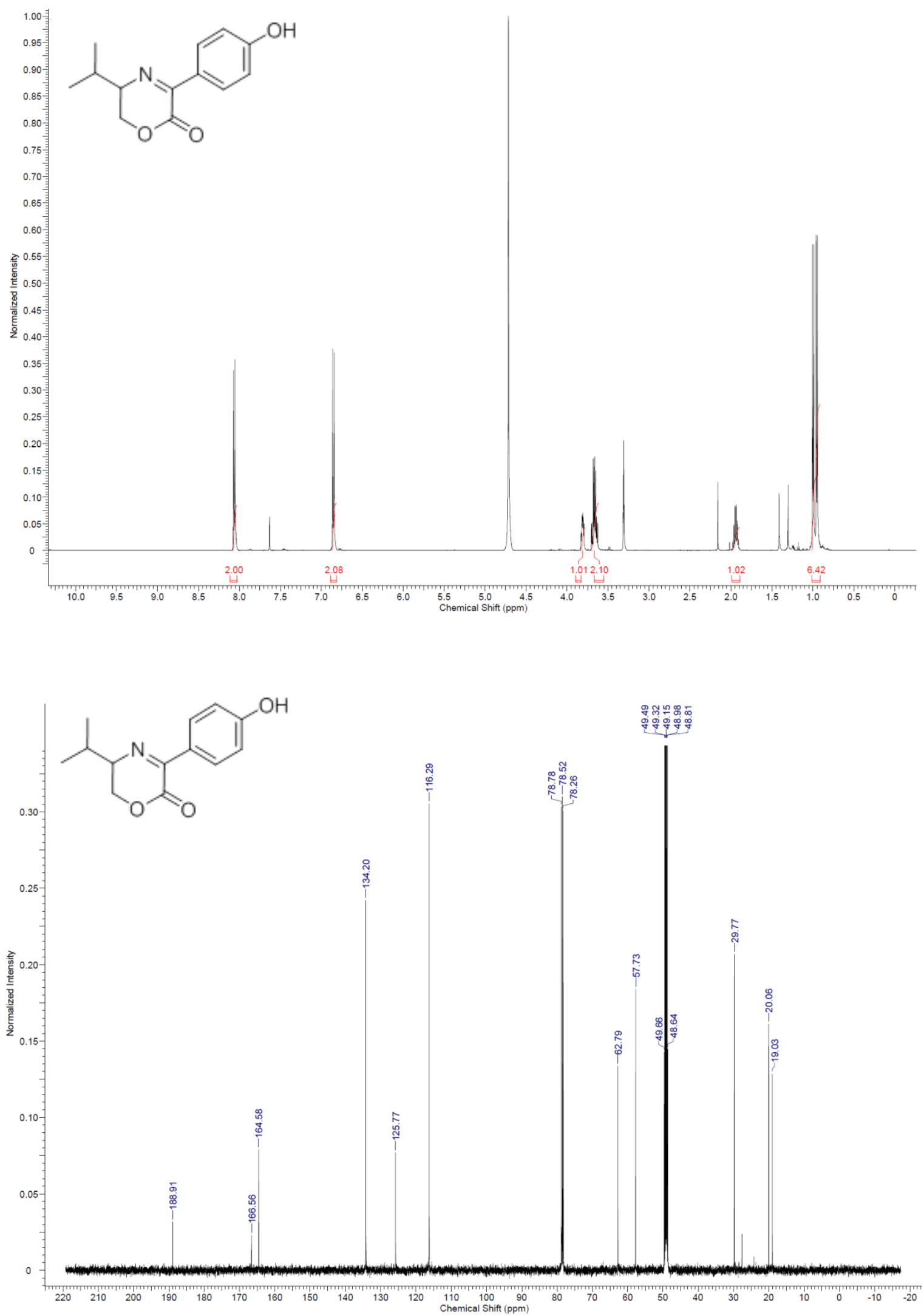


\section{Resin 9a}

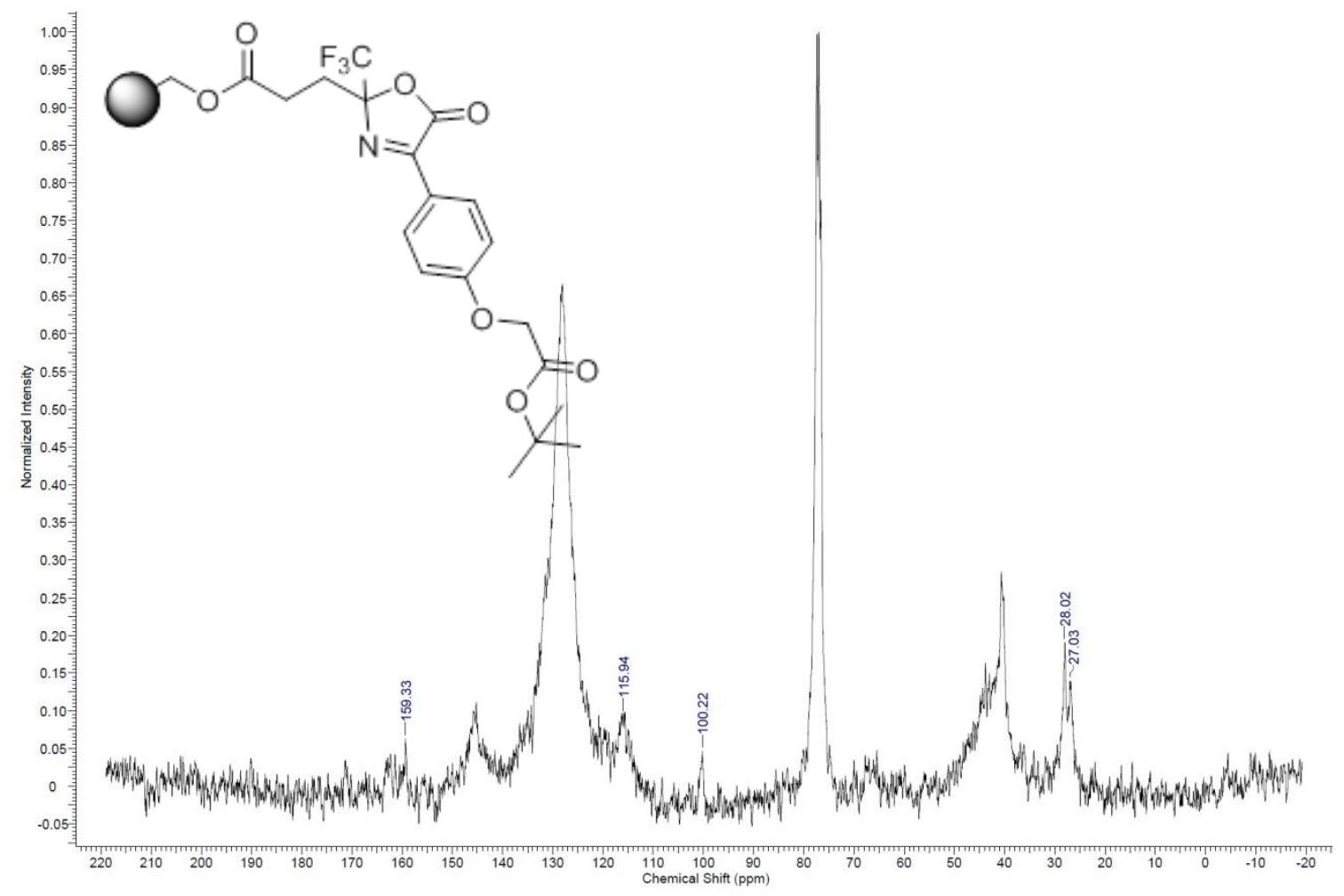

\section{Resin 9b}

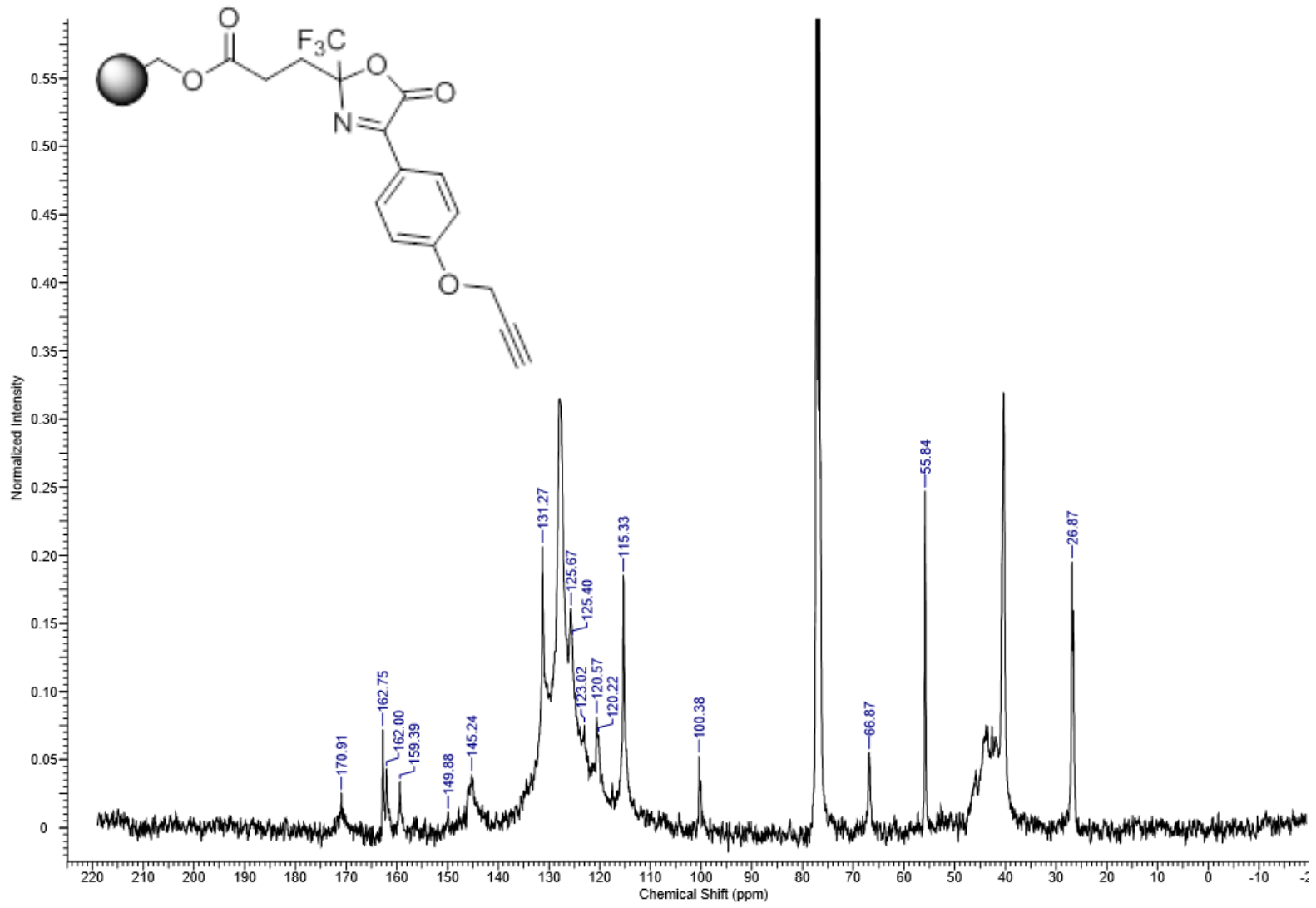


Raman Spectra compound 9b (alkyne band at $2126 \mathrm{~cm}-1$ )

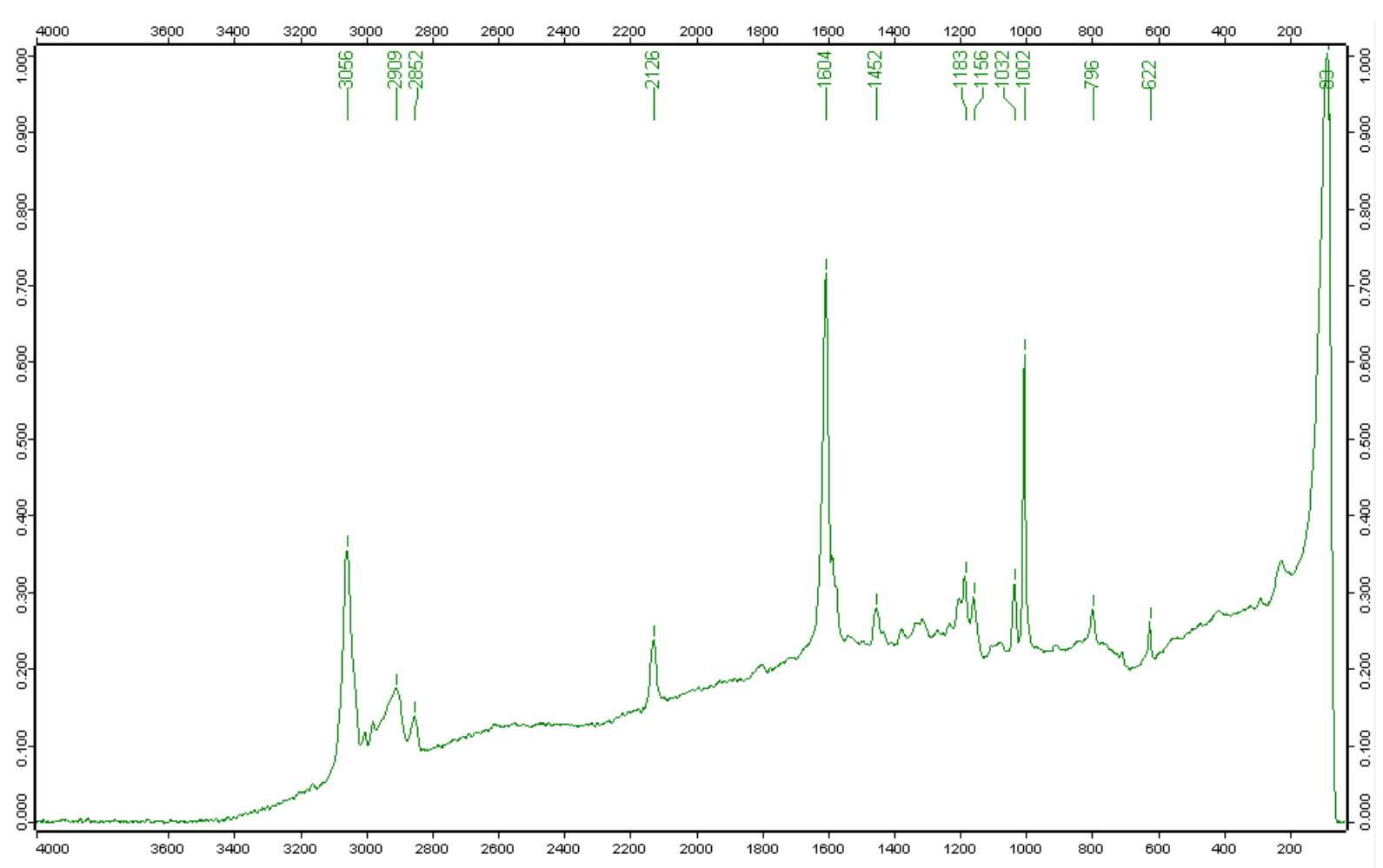

Resin 10

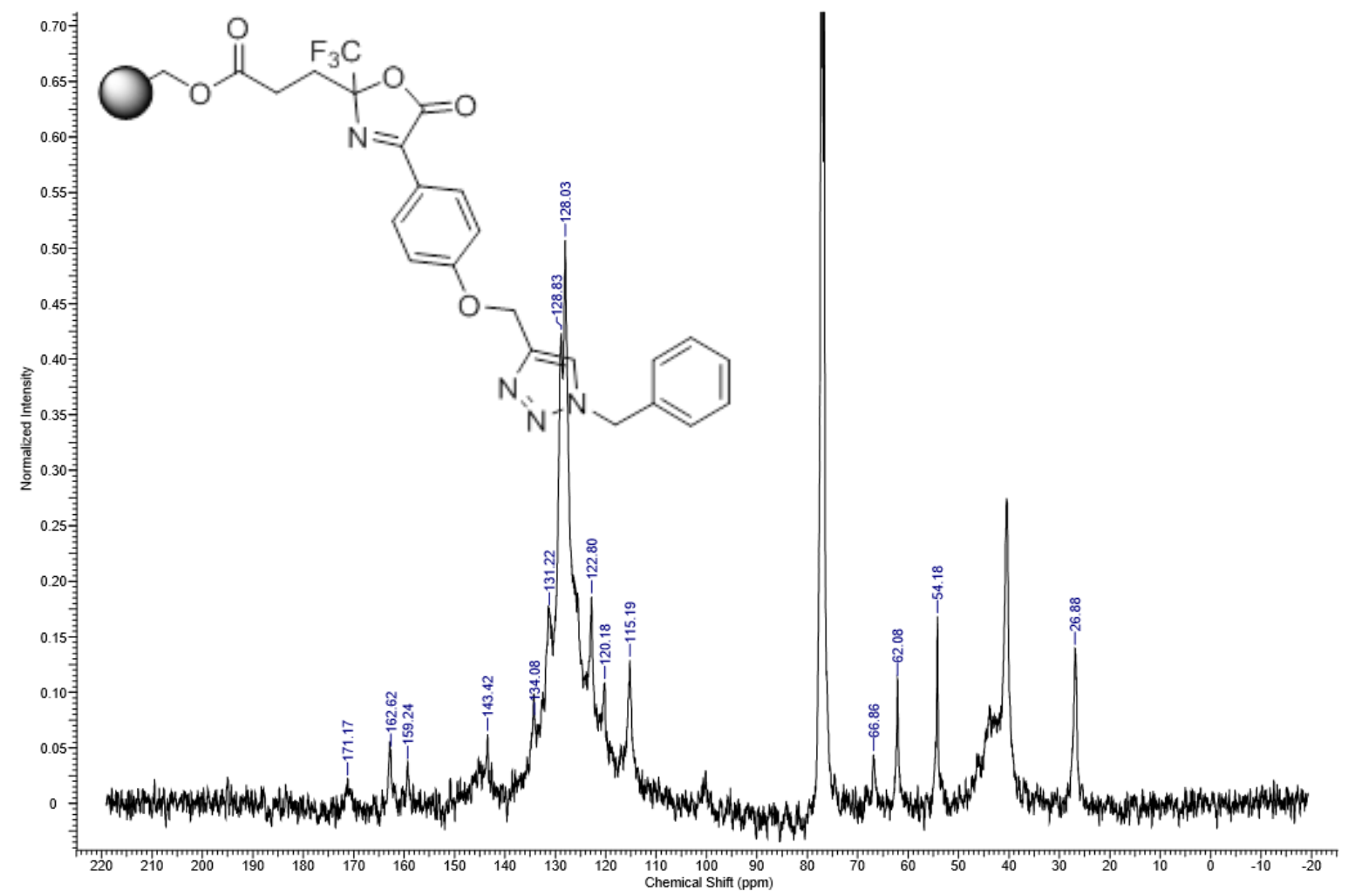


Raman Spectra compound 10 (alkyne band at $2126 \mathrm{~cm}-1$ disappeared)

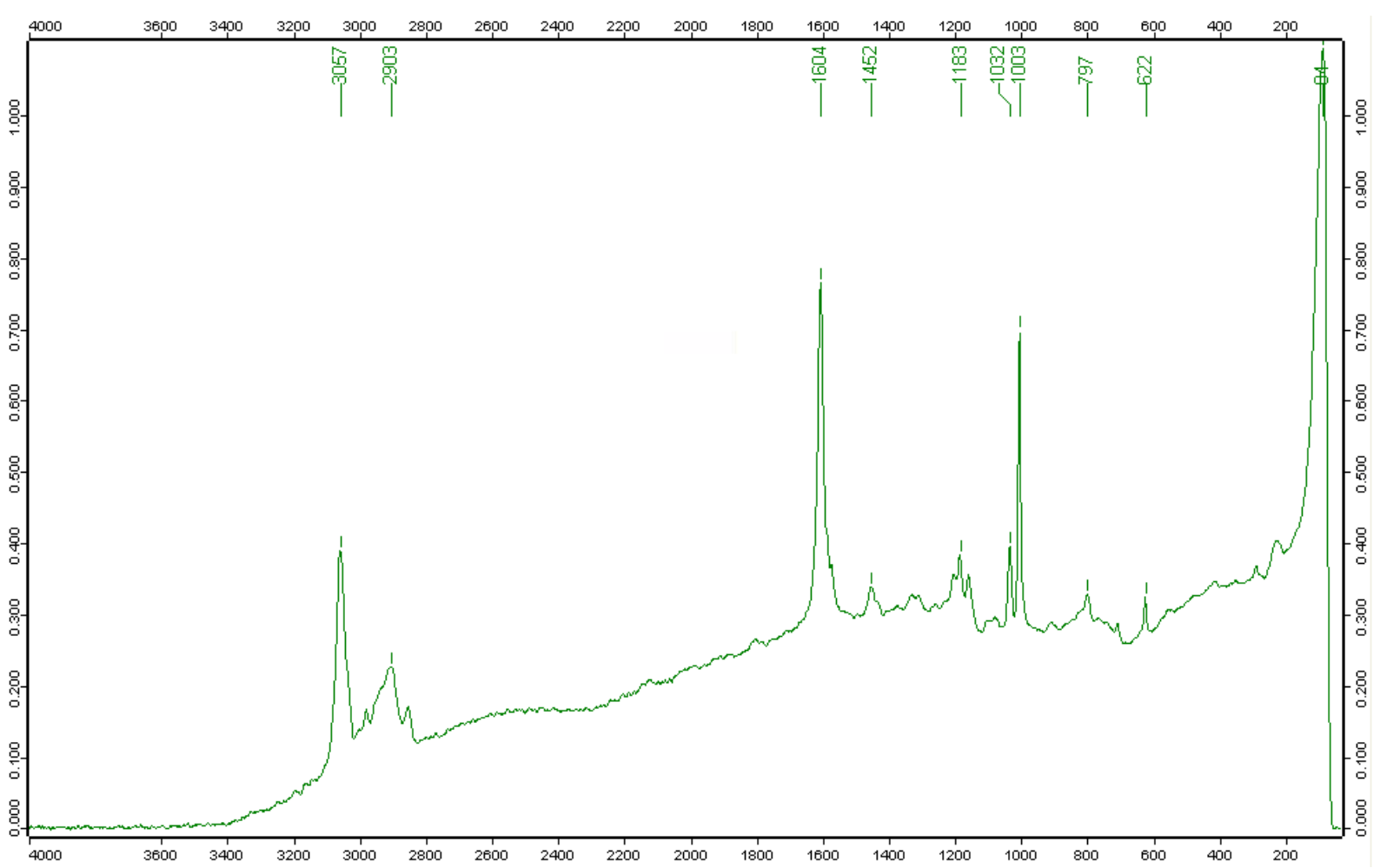


Compound 11a
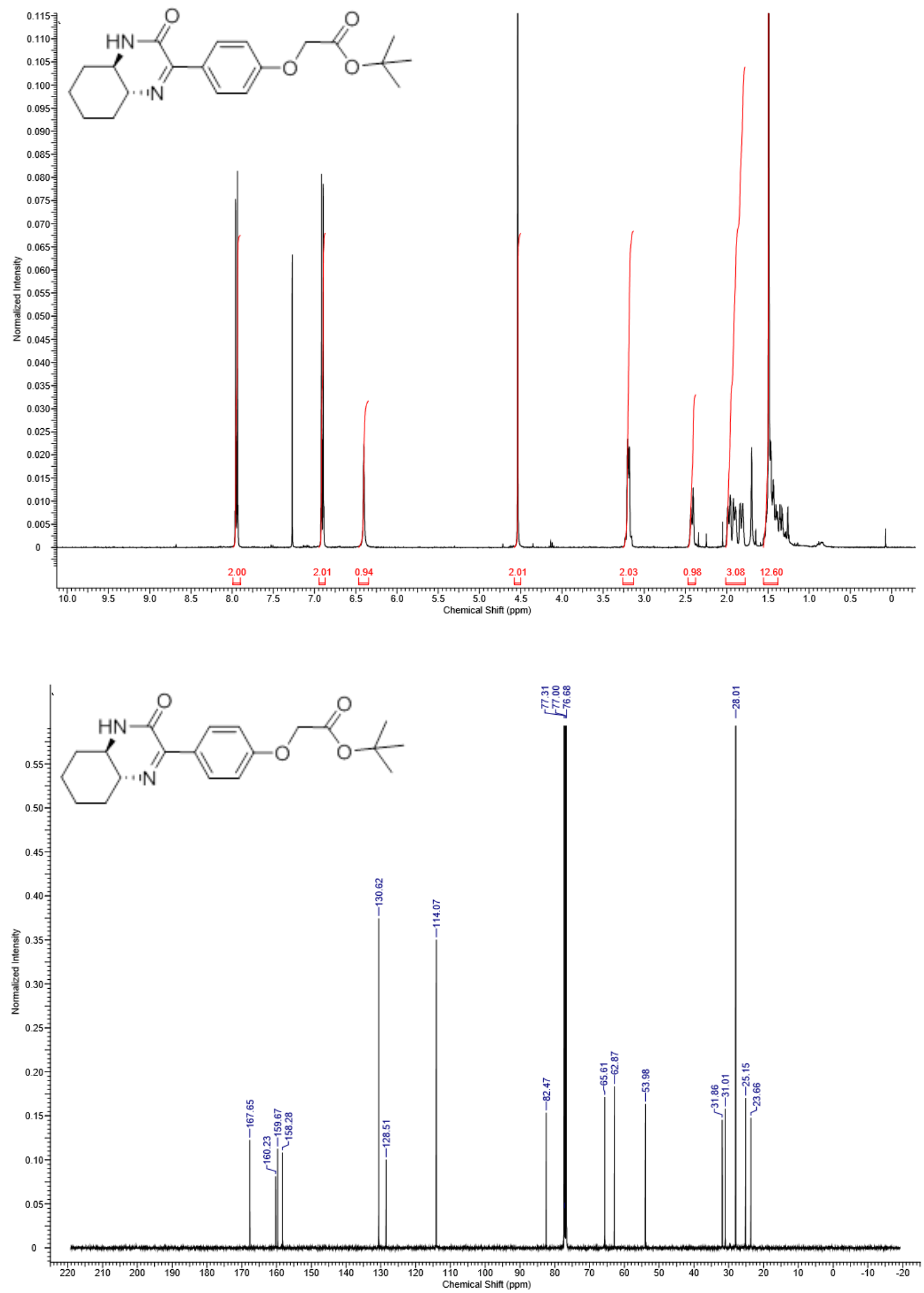


\section{Compound 11b}
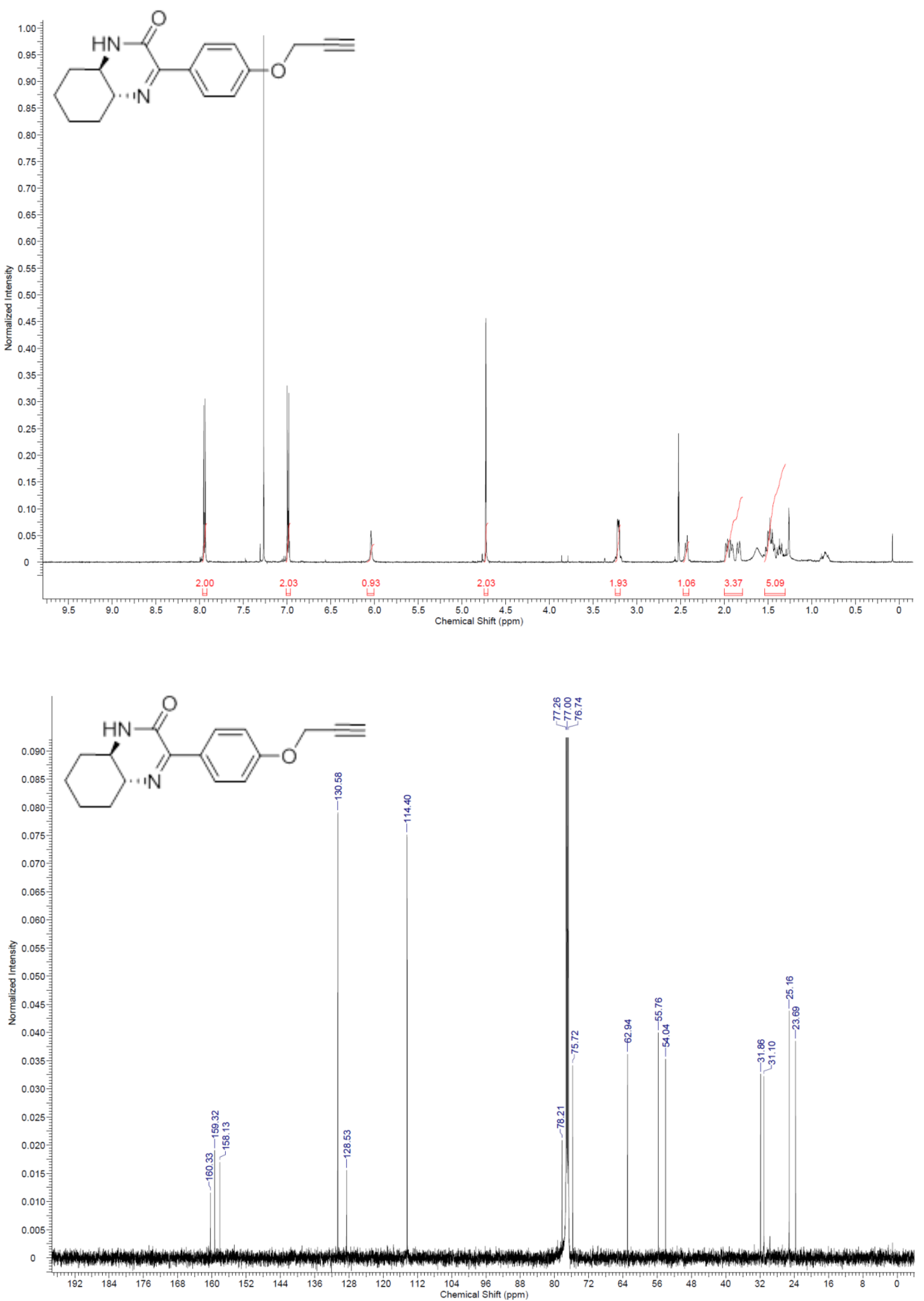


\section{Compound 11c}
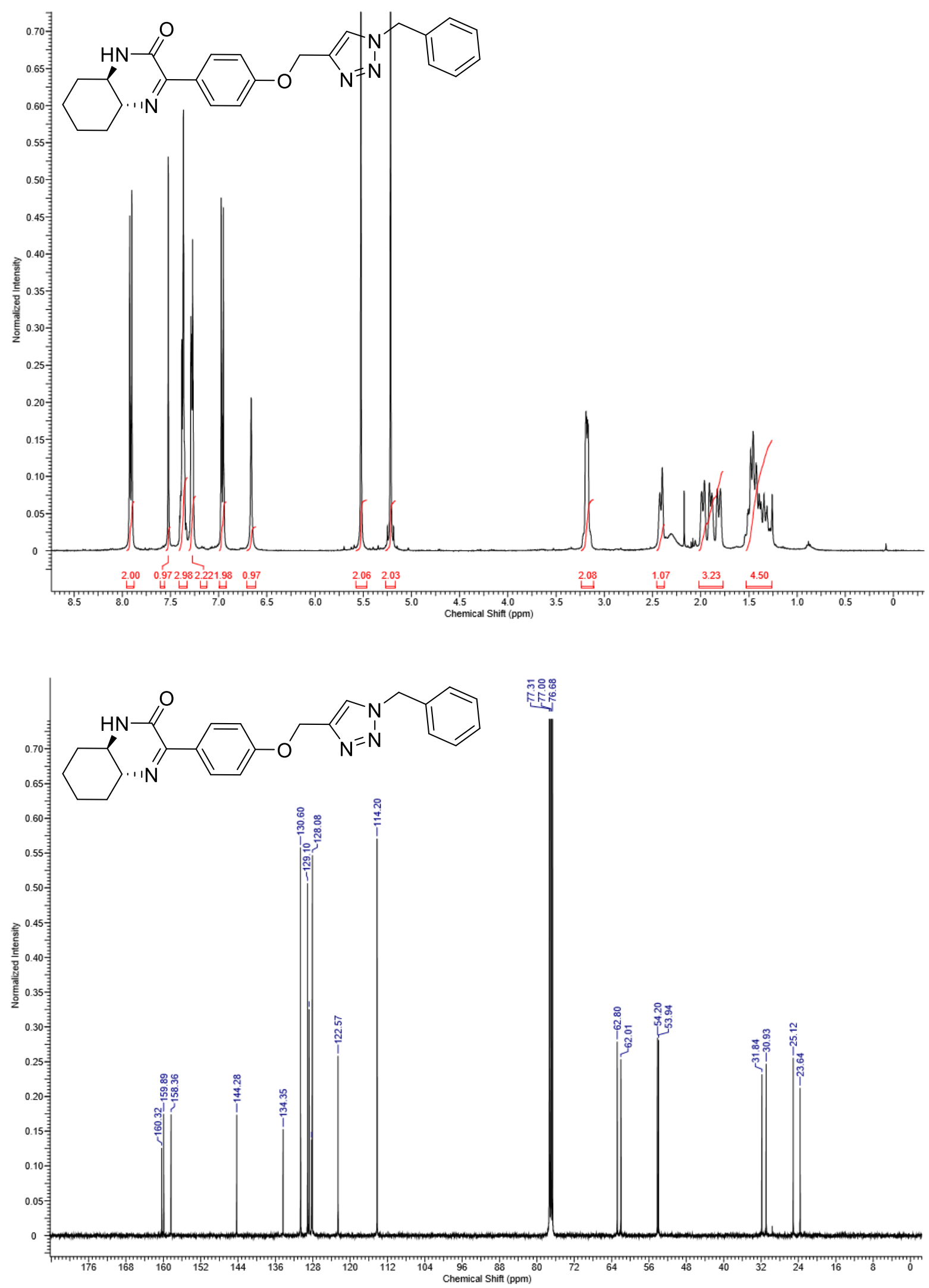